\title{
A SURVEY OF IRRADIATED PILLARS, GLOBULES, AND JETS IN THE CARINA NEBULA
}

\author{
P. Hartigan ${ }^{1}$, M. Reiter ${ }^{2}$, N. SMith ${ }^{2}$, AND J. BALly ${ }^{3}$ \\ ${ }^{1}$ Rice University, Department of Physics and Astronomy, 6100 S. Main, Houston, TX 77521-1892, USA \\ 2 Department of Astronomy, University of Arizona, Tucson, AZ 85721, USA \\ ${ }^{3}$ Center for Astrophysics and Space Astronomy, University of Colorado, Boulder, CO 80309, USA \\ Received 2014 July 16; accepted 2015 January 10; published 2015 February 12
}

\begin{abstract}
We present wide-field, deep narrowband $\mathrm{H}_{2}, \mathrm{Br} \gamma, \mathrm{H} \alpha,\left[\mathrm{S}_{\mathrm{II}}\right],[\mathrm{O} \mathrm{III}]$, and broadband I- and $\mathrm{K}$-band images of the Carina star formation region. The new images provide a large-scale overview of all the $\mathrm{H}_{2}$ and $\mathrm{Br} \gamma$ emission present in over a square degree centered on this signature star-forming complex. By comparing these images with archival Hubble Space Telescope and Spitzer images we observe how intense UV radiation from O and B stars affects star formation in molecular clouds. We use the images to locate new candidate outflows and identify the principal shock waves and irradiated interfaces within dozens of distinct areas of star-forming activity. Shocked molecular gas in jets traces the parts of the flow that are most shielded from the intense UV radiation. Combining the $\mathrm{H}_{2}$ and optical images gives a more complete view of the jets, which are sometimes only visible in $\mathrm{H}_{2}$. The Carina region hosts several compact young clusters, and the gas within these clusters is affected by radiation from both the cluster stars and the massive stars nearby. The Carina Nebula is ideal for studying the physics of young $\mathrm{H}$ II regions and photodissociation region (PDR), as it contains multiple examples of walls and irradiated pillars at various stages of development. Some of the pillars have detached from their host molecular clouds to form proplyds. Fluorescent $\mathrm{H}_{2}$ outlines the interfaces between the ionized and molecular gas, and after removing continuum, we detect spatial offsets between the $\mathrm{Br} \gamma$ and $\mathrm{H}_{2}$ emission along the irradiated interfaces. These spatial offsets can be used to test current models of PDRs once synthetic maps of these lines become available.
\end{abstract}

Key words: H II regions - Herbig-Haro objects - ISM: bubbles - ISM: jets and outflows - photon-dominated region (PDR) - stars: formation

\section{INTRODUCTION}

Most stars form within large molecular cloud complexes that are also sites of massive star formation. Massive stars have profound effects on their parent clouds, primarily by emitting copious amounts of extreme ultraviolet radiation that creates large bubbles of ionized gas which then expand to produce blisters as the gas emerges from the confines of the cloud. Along the edges of the ionized volumes, far-ultraviolet radiation impinges upon the dark clouds, heating and dissociating the molecular gas in a photodissociation region (PDR, a.k.a. photon-dominated regions; e.g., Hollenbach \& Tielens 1997). As the molecular cloud evaporates, the PDR boundary can develop pillars, which later detach to create isolated globules, often with a young star located near the apex of the globule (e.g., O'Dell et al. 1993; Hester et al. 1996). Because PDR boundaries trace surfaces where massive stars deposit energy back into their nascent clouds, following how these boundaries evolve with time is important from the standpoint of star formation energetics. PDRs also provide examples of interfaces between hot ionized gases and cooler, neutral gases where dynamical instabilities may develop.

Because molecular clouds are shrouded in dust, extinction may completely hide PDRs from view at optical and UV wavelengths. Fortunately, $\mathrm{H}_{2}$ fluorescence makes it possible to observe PDR interfaces directly in the near-infrared (NIR) where extinction is an order of magnitude lower. Figure 1 summarizes the basic physics of this interaction. FUV radiation from high-mass stars excites $\mathrm{H}_{2}$ into the Lyman and Werner bands, and subsequent cascades generate strong $2.12 \mu \mathrm{m}$ quadrupole line emission from the $v=1-0, J=3-1$ transition. Hence, the 1-0 S(1) line defines the PDR interface, allowing us to observe precisely where the FUV radiation is absorbed.
Different regions of star formation possess distinct kinds of irradiated interfaces. For example, although the Carina Nebula (and to a lesser extent the Eagle Nebula) have a rich array of pillars, globules, and walls, the more massive but significantly older Cyg OB2 association has mostly isolated globules, while Ara OB1 shows a single long wall (Hartigan et al. 2012). These variations arise from the combined effects of differing ages, number and location of massive stars, and density structures within the parent molecular clouds.

Located $2.3 \mathrm{kpc}$ from the Sun (Smith 2006a), Carina is an ideal target to study firsthand how stars form in the presence of strong radiation. The region contains $\gtrsim 70$ O-type stars (Smith 2006b), and as such is more representative of a typical $\mathrm{H}$ II region we might observe in another galaxy (as opposed to regions like Orion that have $\lesssim 10 \mathrm{O}$ stars). Carina is also young enough to have much ongoing star formation, but old enough that the most massive stars have cleared away enough material to reveal a spectacular naked-eye $\mathrm{H}_{\text {II }}$ region replete with a dizzying array of globules and pillars. Carina spans more than a degree on a side, but with modern imaging systems it is now possible to acquire high-resolution large-scale images that cover the entire star-forming region in a single observing run. Some of the most massive stars in our galaxy are located in Carina, including spectral types as early as O2 (Walborn et al. 2002), WNH stars (Smith \& Conti 2008), and of course, the famous LBV star Eta Car, a prime candidate for the next Galactic supernova.

Carina provides a snapshot of the early phases of an $\mathrm{OB}$ association. Its massive O-type stars are distributed in several clusters, with the two largest groups, $\operatorname{Tr} 14$ and $\operatorname{Tr} 16$, situated in the central cavity of the nebula. These two groups contain several of the most massive stars in the region, and they 


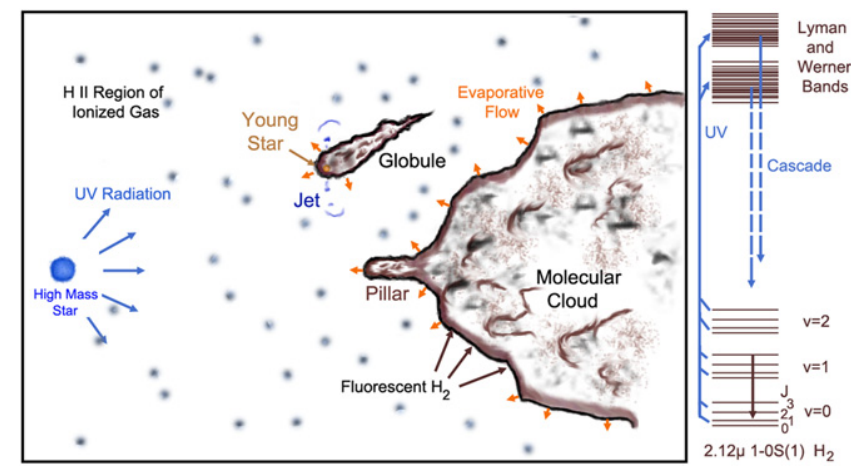

Figure 1. Schematic of a photodissociation region. Ultraviolet radiation from an $\mathrm{O}$-star excites $\mathrm{H}_{2}$ at the interface between an ambient molecular cloud and the $\mathrm{H}$ II region, and the molecule emits $2.12 \mu \mathrm{m}$ line radiation as it transitions to the ground state. Recombination lines of $\mathrm{H} \alpha$ and $\mathrm{Br} \gamma$ form throughout the $\mathrm{H}$ II region, and are enhanced as $\mathrm{H}$ in the dense photoevaporative flow becomes ionized. Globules and pillars form in response to the radiation. Jets from young stars create shock fronts that radiate permitted and forbidden line radiation, and may also excite the $2.12 \mu \mathrm{m}$ lines of $\mathrm{H}_{2}$ if molecules are present within or alongside the jet.

dominate the ionizing flux of the outer Carina Nebula. Tr 16 consists of several sub-clusters identified from X-ray surveys (Feigelson et al. 2011; Wolk et al. 2011), while Tr 14 and the nearby cluster $\operatorname{Tr} 15$ appear more compact. Typical ages for the young stars in $\operatorname{Tr} 14$ and $\operatorname{Tr} 16$ are 1-3 Myr (Ascenso et al. 2007; Hur et al. 2012), with some evidence that $\operatorname{Tr} 15$ is older by several million years (Wang et al. 2011). A substantial fraction of the O-type stars (about half, depending on whether or not one counts $\mathrm{Cr} 228$ as part of $\operatorname{Tr} 16$; see Smith 2006a) are scattered across 30-40 pc residing in several smaller sub-clusters ( $\operatorname{Tr} 15, \mathrm{Cr} 228, \mathrm{Cr} 232$, Bo 10, Bo 11, and others). The presence of multiple groups of massive stars make it difficult to determine which source or sources are responsible for ionizing any given feature within the Nebula.

Historically, the Carina Nebula was considered to be an evolved $\mathrm{H}_{\text {II }}$ region with little evidence for active star formation, a conclusion based on initial far-IR surveys that targeted the inner nebula (Harvey et al. 1979; Ghosh et al. 1988). The first wide-field infrared (IR) survey that revealed the large extent of ongoing star-formation activity was the Midcourse Space Experiment (MSX) imaging of Carina (Smith et al. 2000). These wide-field mid-IR images uncovered an extended network of pillars, globules, bubbles, and embedded IR sources spread across several degrees. A number of other studies focused on signs of star formation and bright individual PDRs in the region (Megeath et al. 1996; Brooks et al. 2000, 2001, 2003; Rathborne et al. 2002, 2004; Smith et al. 2004b; Smith et al. 2005). Smith \& Brooks (2007) provide an overview of the global multiwavelength structure and energy budget of Carina. Later, imaging with the Spitzer Space Telescope clarified much of the spatial relationship between stars, ionized gas, polycyclic aromatic hydrocarbons, and dust and also revealed thousands of embedded protostars and disk excess sources organized in a hierarchical network of young star clusters spread across 30-40 pc (Smith et al. 2010b; Povich et al. 2011a). This collection of young clusters was confirmed with wide-field X-ray images obtained with Chandra, which documented the numerous $\mathrm{T}$ Tauri stars throughout the region (e.g., Feigelson et al. 2011).

Following the first ground-based discovery of a protostellar jet in the region (HH 666; Smith et al. 2004a), a survey with the Hubble Space Telescope (HST) Advanced Camera for Surveys (ACS) revealed $\sim 40$ jets or candidate jets (Smith et al. 2010a), confirming the active ongoing star formation in the region. The HST images included a contiguous mosaic of the region surrounding the $\operatorname{Tr} 14$ and 16 clusters, plus a number of other individual fields in the South Pillars and other regions. Many of these protostellar jets emerge from compact dark globules, also scattered throughout the region, indicating that at least some of these compact globules must contain protostars. Spitzer also revealed a few candidate molecular outflows based on their excess emission in the IRAC Band 2 filter (Smith et al. 2010b). We expand the census of known outflow sources with the wide-field images in this paper.

Several recent papers have explored the Carina star-forming region with large surveys at both X-ray, IR, and submillimeter wavelengths. As part of the Chandra Carina Complex Project (CCCP; Broos et al. 2011; Townsley et al. 2011), the Carina Nebula was targeted for deep X-ray exposures, and over 14,000 point sources were discovered. In the NIR, wide-field JHK images of the central portion of the Carina complex (Preibisch et al. 2011a, 2011b) with the Very Large Telescope showed that most of the X-ray sources were likely to be cluster members. In the submillimeter, Preibisch et al. (2011c) and Pekruhl et al. (2013) surveyed an area of the Carina Nebula with 18 arcsec resolution over a square degree and identified all of the principal areas of warm dust in the region, while Roccatagliata et al. (2013) published far-IR maps of the entire region using Herschel with similar spatial resolution. In the optical, Grenman \& Gahm (2014) compiled a list of dozens of small dark globules in Carina from archival HST $\mathrm{H} \alpha$ images of the central portion of the Carina star formation region. Tapia et al. (2011) has published $\mathrm{H}_{2} 2.12 \mu \mathrm{m}$ images of selected objects within Carina, and Ohlendorf et al. (2012) discuss midIR Spitzer images of the region whose filters include lines of $\mathrm{H}_{2}$.

Carina, with its strong UV field, modest extinction, and plentiful ongoing star formation is the premier region for studying how ionizing radiation affects jets (e.g., Smith et al. 2010a; Ohlendorf et al. 2012). Once jets emerge into the $\mathrm{H}$ II region, they become photoionized by the ambient ultraviolet light. Cooling from this process complicates the interpretation of emission line images because the observed radiation arises from both shock heating and radiative heating, and it can be difficult to distinguish a photoionized filament from a photoionized jet. In fact, Reiter \& Smith (2013) found that most of the $\mathrm{H} \alpha$ and $\left[\mathrm{S}_{\mathrm{II}}\right]$ emission from several Carina jets traces ionization fronts that arise from external UV radiation. However, there is also evidence for shielding, in that the dense central parts of jets appear relatively unaffected by the ambient radiation field when they first emerge from their natal cloud cores. In a region as complex as Carina, searches for jets benefit from molecular tracers such as $\mathrm{H}_{2}$ to unveil embedded outflows (e.g., Stanke et al. 2000, 2002; Smith et al. 2010b; Lee et al. $2012,2013)$ in addition to the standard optical tracers of $\mathrm{H} \alpha$ and $[\mathrm{S}$ II $]$.

In this paper we compile the deepest $\mathrm{H}_{2}$ and $\mathrm{Br}-\gamma$ mosaics to date of the Carina star-forming region and compare them with new narrowband optical and broadband NIR images. The images cover three times the area of the JHK mosaics of Preibisch et al. (2011a), and reveal subarcsecond detail along dozens of interfaces where FUV radiation from massive stars is in the process of destroying ambient molecular clouds. By 
subtracting appropriately scaled continuum images from the narrowband ones, we construct pure emission-line images of the irradiated interfaces. The observed spatial offsets between the $\mathrm{Br} \gamma$ and $\mathrm{H}_{2}$ emission along the PDRs are independent of the reddening, and quantify how quickly the molecular gas flows into the $\mathrm{H}$ II region. Shocked $\mathrm{H}_{2}$ gas delineates jets while they are embedded deep within a dark cloud, and complements the spatial structure of jets inferred from $\mathrm{H} \alpha$ and $\left[\mathrm{S}_{\mathrm{II}}\right]$ emission (cooling zones of shocks and irradiated flows). Our [O III] images show the most highly ionized parts of pillars, identify the highest shock velocities, and help to quantify how ambient UV-radiation fields affect shocked gas.

A main focus of this paper is to identify all the observable outflows and irradiated interfaces in Carina that are visible in our images, with the aim to provide a snapshot of the walls, pillars, globules, clusters, jets, and so on that are present within a typical region of ongoing massive star formation. Numerical simulations which include magnetic fields and radiative transfer (e.g., Arthur et al. 2011) can use the examples shown here to compare with the types of structures present in the models in order to better understand how star formation evolves in real systems.

\section{OBSERVATIONS}

\section{1. $\mathrm{H}_{2}, \mathrm{Br} \gamma$, and $\mathrm{K}$ Mosaics}

The NIR images of Carina in this paper were taken 2011 March 11-18 with the NEWFIRM camera at the NOAO Blanco $4 \mathrm{~m}$ telescope at Cerro-Tololo in Chile. NEWFIRM is a large-format imager that consists of four $2048 \times 2048 \mathrm{InSb}$ arrays (Probst et al. 2008). A single exposure covers 27.6 arcmin with a gap of 35 arcsec between the arrays at a scale of 0.4 arcsec pixel ${ }^{-1}$. Images were taken in $J, H, K$, and through narrowband filters centered at $\mathrm{H}_{2} 1-0 \mathrm{~S}(1)$ and $\mathrm{Br} \gamma$ using the standard NEWFIRM filter set. The $\mathrm{H}_{2}$ and $\mathrm{Br} \gamma$ filters both have $\Delta \lambda / \lambda$ of $\sim 1.1 \%$. All mosaics were reduced and analyzed within the IRAF environment.

Because there are gaps between the arrays as well as numerous cosmetic defects, the usual strategy for observing with NEWFIRM is to obtain at least several dozen dithered exposures of a single field and use software to combine the set of images into a single mosaic; NOAO has devised a pipeline reduction process to perform this task. Unfortunately the pipeline does not work well for regions such as Carina that have a large amount of extended emission, because the pipeline interprets the extended emission as enhanced background sky, and subtracts it. As a result, artificial negative "bowls" surround all regions of extended emission in the pipeline reductions.

To solve this problem we observed $\mathrm{Br} \gamma$ and $\mathrm{H}_{2}$ in $6 \times 6$ grids with two small (<1 arcmin) random dithers for each grid point and employed offsets between adjacent grid points of typically 6 arcmin. With this strategy each point in the mosaic is observed enough times to eliminate bad pixels, and the grid covers a wide enough area so that each array has a substantial number of exposures that have no objects with extended emission, thereby eliminating sky-subtraction biases. However, this procedure demands that the sky exposures are always free of extended emission, so it is not possible to use the pipeline default of a group of exposures nearest in time. We tested our procedure by using a more time-consuming method of offsetting 1.5 away for sky during the mosaic. Such large offsets proved problematic as they introduced significant positional drift during the mosaic, but the result was similar to what we were able to achieve with our $6 \times 6 \times 2$ mosaic.

Exposure times at each pointing were 2 minutes, so the entire exposure time for a single mosaic is 72 minutes. Allowing for dithers, telescope settling, and readout, each mosaic takes about $2 \mathrm{hr}$ to complete in real time. The actual exposure time for a given location in the mosaic is largest near the center of the mosaic, and decreases toward the edges because fewer images contribute to the final mosaic at those locations. Nights with the best seeing were used for the $\mathrm{H}_{2}$ and $\mathrm{Br} \gamma$ observations. The sky was stable enough during the exposure sequence to enable sky subtraction between any two images in a given mosaic. Eliminating the mosaics with the worst seeing, we were left with 4 mosaics in $\mathrm{Br} \gamma$ and 6 mosaics in $\mathrm{H}_{2}$. Combining these produced the final mosaic for each filter. The resulting $\mathrm{H}_{2}$ and $\mathrm{Br} \gamma$ mosaics have excellent image quality (FWHM images of 0.74 and 0.79 arcsec, respectively), each span about 1.1 on a side, and have central exposure times of $3.5 \mathrm{hr}$ for $\mathrm{H}_{2}$ and $2.4 \mathrm{hr}$ for $\operatorname{Br} \gamma$ (Table 1). Distortion corrections and final coordinate mapping for the NEWFIRM images are determined by the pipeline process, and make use of known positions of bright stars in the 2MASS catalog. A check of the coordinates for several of the brightest stars in the final mosaics against their position in the SIMBAD database shows errors $\lesssim 1$ pixel (0.4 arcsec).

We also imaged Carina in $J, H$, and $K$, with the aim of establishing a photometric database for the region, and as an aid to subtracting ambient continuum radiation as described below. Large-scale nebular emission is less of a problem for these filters, so we could acquire them using small mosaics under less favorable weather conditions, and later combine them to produce full mosaics. As such, the image quality in the $J, H$, and $K$ band mosaics, while typically 0.7 arcsec, varies across the field of view and approaches 2 arcsec in a few areas.

Interpreting narrowband images of regions like Carina can be problematic because reflected light from dust may be present along the same interfaces that produce $\mathrm{H}_{2}$ and $\mathrm{Br} \gamma$ emission lines. However, because the wavelengths of $\mathrm{H}_{2}$ and $\mathrm{Br} \gamma$ are nearly the same, the reddening is essentially identical at each point in the two images; hence, the continuum can be removed well by simply forming a scaled difference image $\left(\mathrm{H}_{2}-\right.$ $\mathrm{C} \times \mathrm{Br} \gamma$ ), where the scale factor $C$ takes into account the differences in the filter bandpass widths. The NEWFIRM filter bandpasses imply $C=1.19$ for flat continuum sources. To verify this number we performed photometric measurements on a sample of stars in the final mosaics and found $C=1.19 \pm$ 0.05. All $\mathrm{H}_{2}-\mathrm{Br} \gamma$ difference images shown in this paper include this correction factor. While extended continuum subtracts out well in the $\mathrm{H}_{2}-\mathrm{Br} \gamma$ composites, stellar images typically leave a dark core surrounded by a light halo because the seeing was slightly better in the final $\mathrm{H}_{2}$ composite than it was in $\operatorname{Br} \gamma$. Stars that have strong $\operatorname{Br} \gamma$ emission will appear white in the difference images.

The $\mathrm{H}_{2}-\mathrm{Br} \gamma$ difference images are very effective for showing gradients in PDRs. However, quantifying the spatial offsets between the emission lines requires comparing spatial profiles across the two narrowband images, and these profiles include continuum. In principle one can remove the continuum algebraically by using the $\mathrm{H}_{2}, \mathrm{Br} \gamma$, and $K$ band filters, scaling from their respective bandpasses and noting that any $\mathrm{H}_{2}$ and $\mathrm{Br} \gamma$ emission lines also contribute to the $K$ band flux 
(essentially three equations and three unknowns at each point in the image). While mathematically correct, the different seeing in the three mosaics leads to poor subtractions of point sources and distracting residuals at the locations of the stars. Hence, we have performed this procedure only along the edges of pillars and bright PDR interfaces where we measure the spatial offsets between $\mathrm{H}_{2}$ and $\mathrm{Br} \gamma$ (see Section 3.2).

\section{2. $H \alpha,\left[S_{I I}\right],\left[O_{I I I}\right]$, I-band, and Spitzer IRAC Mosaics}

We obtained narrow-band images of the Carina Nebula on 2003 March 9-11 using the $8192 \times 8192$ pixel Mosaic 2 imager mounted at the prime focus of the CTIO $4 \mathrm{~m}$ Blanco telescope. This camera has a pixel scale of 0.27 pixel $^{-1}$ and a 35.4 field of view. We used $\Delta \lambda=80 \AA$ interference filters centers on the $\lambda=5007$ [O III] line, the $\lambda=6563 \mathrm{H} \alpha$ line, and the $\lambda \lambda=6717 / 6731$ [S II] doublet, and a broadband filter transmitting the SDSS $i$ band. In each filter, we took several individual exposures with slight positional offsets to correct for gaps in the CCD array and to correct for detector artifacts.

We reduced the data in the standard fashion with the MSCRED package in IRAF, and absolute sky coordinates were computed with reference to USNO catalog stars. Emission-line images were flux calibrated by comparison with images of the Orion Nebula obtained during the same run, and the Orion images were calibrated with reference to HST data. Distortion corrections for the Mosaic images were determined by measuring $\sim 100$ stars in common between the optical and NEWFIRM images, resulting in alignment errors $\sim 0.1$ arcsec rms between the optical and IR images.

In this paper we also make use of existing Spitzer images of the region. Smith et al. (2010b) targeted two regions in the Carina Nebula with Spitzer for deep imaging in order to search for lower-mass protostars $\left(1-3 M_{\odot}\right)$. Based on extended midIR emission in lower-resolution images from the MSX survey, those authors observed the South Pillars to further study the complex molecular emission and evidence for current star formation amid a large number of recently formed stars. The western region was chosen as an edge-on view of an ionization front and PDR that also shows signs of star formation. These data were reduced using the GLIMPSE pipeline, and are described in more detail in Smith et al. (2010b).

In the following sections we consider the PDR interfaces as defined by $\mathrm{H}_{2}$ fluorescence, and then turn to what the NEWFIRM and MOSAIC images reveal about outflows.

\section{OBSERVATIONS OF PDR INTERFACES}

Figure 2 identifies 61 areas of interest in the $\mathrm{H}_{2}$ and $\mathrm{Br} \gamma$ mosaics that are reproduced in Figures 3-14. Each area has some some notable aspect of $\mathrm{H}_{2}$ or $\mathrm{Br} \gamma$ emission, in the form of a spatially resolved irradiated surface, a jet, cavity, bow shock or distinct nebular feature. These areas are located in one of eight larger regions in Carina: the Southern Pillars (Figures 3 and 4), the Eastern Walls (Figures 5-7), the Northern Pillars (Figures 8 and 9), the vicinity of $\operatorname{Tr} 14$ (Figure 10), a region around $\eta$ Car (Figure 11), the Southwestern Pillars and Walls (Figure 12), the Northwestern Pillars and Walls (Figure 13), and the Western Wall (Figure 14). In what follows we collect the various types of objects together and refer to them by their area number.

\subsection{Irradiated Walls, Pillars, and Globules}

Several molecular "walls" of irradiated $\mathrm{H}_{2}$ with lengths $1-5 \mathrm{pc}$ exist within the boundaries of the survey. As expected, pillars and waves along these walls form on the sides that face the ionizing sources in the center of mosaic, though it is difficult to assign a definitive exciting source owing to the large number of $\mathrm{O}$ stars in the region. Overplotting the 94 candidate OB stars of Povich et al. (2011b) on the $\mathrm{H}_{2}$ composite did not yield any insights into the identities of the exciting sources.

The walls generally divide into three categories: (1) structured walls with few or no pillars as exist in Areas 5, 9, 16, 46, 47, 51, and 56; (2) structured walls with "chunky" pillars that have aspect ratios $1 \sim 2$ in Areas 3, 7, 10, 11, 14, 33, 44, 48, 49, 52, 53, 59, 60, and 61; and (3) structured walls with well-defined pillars such as those in Areas 22, 27, 29, 32, 44 , and 50. There does not seem to be any obvious relationship between the (projected) distance of the walls from $\operatorname{Tr} 14$ and $\mathrm{Tr}$ 16 and the presence of pillars. For example, pillars exist along the walls in both Areas 29 and 44, the former situated only about $1.5 \mathrm{pc}$ from $\operatorname{Tr} 14$, while the latter lies at the western extreme of the $\mathrm{H}_{2}$ emission a bit more than $10 \mathrm{pc}$ away in projection from both $\operatorname{Tr} 14$ and $\operatorname{Tr} 16$.

The pillars come in a variety of aspect ratios, ranging from those with rounded heads and aspect ratios $\sim 5$ (Areas 1, 3, 8, $14,15,17,18,27,31,45$, bottom of Area 22) to pillars that are very long and narrow (Areas 2, 4, 21, 23, 24, 37, top-right corner of Area 10). It is common for pillars to exhibit multiple arcs along their sides, implying a shouldered or scalloped shape (Areas 1, 2, 4, 18, 20, 21, 27, 33, 45). Some pillars have one one or more bright NIR continuum sources situated near their apices (Areas 2, 3, 4, 8, 18, 21, bottom of Area 10), while others do not (Area 1, 14, 17, bottom of Area 22). There are many background and foreground stars in the region that could by chance coincide with the apex of any given pillar. However, in some cases (e.g., Area 4) enhanced $\mathrm{Br}-\gamma$ emission at the location of the NIR source associates the source with the pillar.

Isolated irradiated globules and pillars that have detached from their parent clouds exist throughout the region, and abound in the vicinity of Eta Car and Tr 14. Some of these show a classic head-tail "proplyd" shape (Areas 23 and 24), while others are much more irregular (Areas 25 and 28). Several of these objects have one or more bright NIR continuum sources near their apices (Area 6, 21, 23, 24, 25, 40, 41, 55, upper left corner of Area 15), while others do not show any obvious NIR continuum sources near the head of the pillar (Area 12, 28, 32, 35, 36, 38, 39, 54, the object to right of main pillar in Area 4, and small globules in Area 6). In many cases the NIR source at the apex may drive a jet, but these can be difficult to detect at ground-based spatial resolution, and also because in the absence of strong extinction, $\operatorname{Br} \gamma$ is considerably fainter than $\mathrm{H} \alpha$ for both shocked and irradiated flows. For example, Smith et al. (2010b) identified the star at the southern end of the pillar in Area 24 of Figure 10 as the driving source for the HH 1013 flow they discovered with HST ACS images, but these $\mathrm{HH}$ objects are too small to resolve against the variable background in our ground-based images.

A collection of $\mathrm{H}_{2}$ emission features in Figure 2 in the Eastern Wall region that extends from Area 13 through Area 14 in Figure 6 has some unusual characteristics. Unlike the major pillars highlighted above, all of which are bright in $70 \mu \mathrm{m}$ Herschel images (Preibisch et al. 2012), these regions of $\mathrm{H}_{2}$ emission are not readily visible with Herschel. However, there 


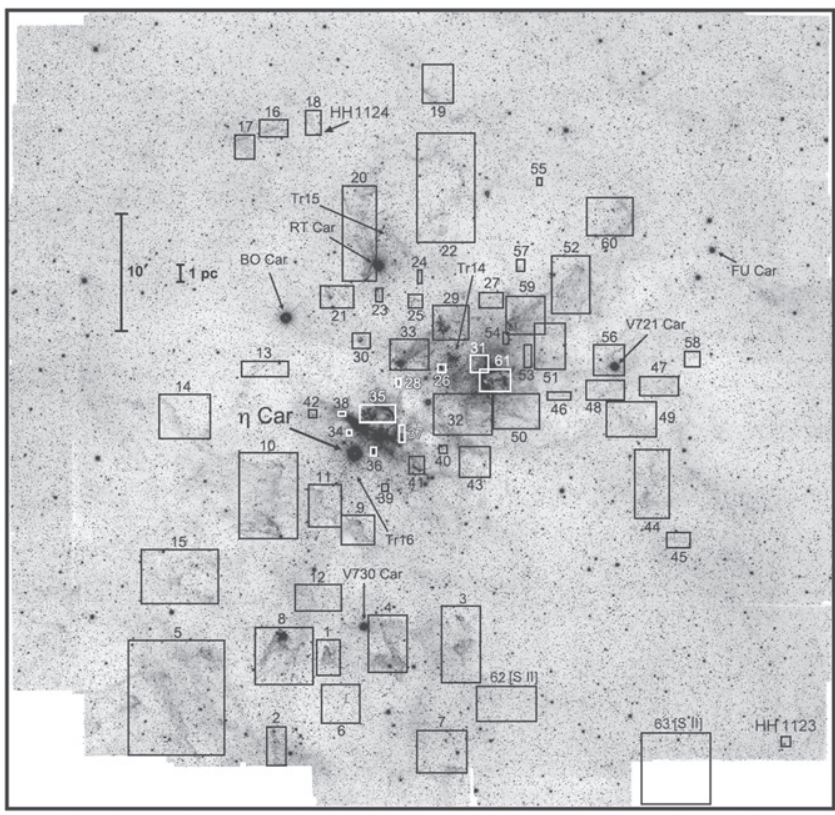

Figure 2. Overview of the Carina region in $\mathrm{H}_{2} 2.12 \mu \mathrm{m}$. Numbered boxes mark the regions of $\mathrm{H}_{2}$ emission highlighted in Figure 3. The scale bar assumes a distance of $2.3 \mathrm{kpc}$. Coordinates for the centers of each box are compiled in Table 2. The clusters $\operatorname{Tr} 14, \operatorname{Tr} 15, \operatorname{Tr} 16$, and the brightest stars are marked. The image spans $\sim 1$. 1 on a side, with a scale of $0.4 \operatorname{arcsec}_{\text {pixel }}^{-1}$. North is up and east to the left in this and subsequent images. radiation field. Both dust and gas serve to shield the molecular cloud from the radiation, but the opacities vary strongly with wavelength at ionization thresholds, and the situation is complicated by photoablation that causes newly ionized gas to flow away from the cloud. Numerical models that include the requisite atomic and molecular radiative transfer as well as dynamics are just becoming available (e.g., Arthur et al. 2011), so quantifying these spatial offsets is a timely endeavor from the observational standpoint, and will hopefully motivate numerical models to predict offsets from their codes.

We chose the spectacular irradiated interface associated with G287.38-0.62 (Retallack 1983; Brooks et al. 2003; the "Western Wall" in Area 61 of Figure 14; see Figure 15 for a color composite) to study emission line offsets in PDRs. This interface is very bright in all emission lines, and is large enough to allow measurements to be made along the periphery of the cloud where the incident ionizing radiation is more oblique, and near the apex where the radiation is more normal to the surface. One complication is that irradiated interfaces emit continuum radiation from reflected starlight. The filter bandpasses for $\mathrm{Br} \gamma$ and $\mathrm{H}_{2}$ have somewhat different widths, and so subtraction between the two images could potentially affect the spatial distribution of the emission. We removed the continuum using the procedure described in Section 2.1.

Intensity profiles extracted along the four line segments shown in Figure 15 appear in Figure 16. We define the position

Table 1

Summary of Carina Mosaics

\begin{tabular}{|c|c|c|c|c|}
\hline Date & Filter & Exposure Time $(\mathrm{ks})^{\mathrm{a}}$ & FWHM $(\operatorname{arcsec})^{\mathrm{b}}$ & Camera \\
\hline 2003 Mar 9-10 & {$[\mathrm{O}$ III $] 5007 \AA$} & 0.60 & 0.8 & Mosaic \\
\hline 2003 Mar 9-10 & $\mathrm{H} \alpha 6563 \AA$ & 0.60 & 0.8 & Mosaic \\
\hline 2003 Mar 9-10 & [S II] $6720 \AA$ & 0.60 & 0.8 & Mosaic \\
\hline 2011 Mar 13-16 & $\mathrm{H}_{2} 2.12 \mu \mathrm{m}$ & 12.5 & 0.7 & Newfirm \\
\hline 2011 Mar $14-17$ & $\operatorname{Br} \gamma 2.16 \mu \mathrm{m}$ & 8.7 & 0.8 & Newfirm \\
\hline 2011 Mar 9-18 & $\mathrm{K} 2.2 \mu \mathrm{m}$ & $\cdots$ & $0.7-2.2$ & Newfirm \\
\hline Jan 21-22, Jun 13, 2005 Jul 17 & $5.8 \mu \mathrm{m}$ & 0.032 & $\sim 2$ & Spitzer \\
\hline Jan 21-22, Jun 13, 2005 Jul 17 & $8.0 \mu \mathrm{m}$ & 0.032 & $\sim 2$ & Spitzer \\
\hline
\end{tabular}

a Typical exposure time at center of mosaic.

${ }^{\mathrm{b}}$ Image quality of final coadded mosaic.

is a good correlation between absorption in our $\mathrm{H} \alpha$ images and these $\mathrm{H}_{2}$ structures, so some dust exists in these regions. The most likely explanation is that we are observing a lowextinction PDR that lacks sufficient dust column density to produce observable far-IR continuum.

\subsection{Spatial Offsets between $\mathrm{H}_{2} \mathrm{l}-\mathrm{O} S(\mathrm{l})$ and $\mathrm{Br} \gamma$}

Throughout the Carina Nebula we consistently observe spatial offsets between $\mathrm{Br} \gamma$ and $\mathrm{H}_{2}$ emission along irradiated interfaces in the sense that $\mathrm{Br} \gamma$ is located closer to the source of ionizing radiation. This spatial offset is typically $\sim 1^{\prime \prime}$, which corresponds to $\sim 20$ times the rms scatter between the stellar positions in the $\mathrm{Br} \gamma$ and $\mathrm{H}_{2}$ images. This offset is independent of the reddening, and a potentially important diagnostic for PDRs. The offset involves a rather complex interplay between the atomic, molecular and ionized gas, the dust, and the of the ionization front as the location where the normalized $\mathrm{H} \alpha$ and $[\mathrm{S}$ II $]$ profiles intersect. In practice, this location is simply a convenient fiducial point. In each panel there is a small offset on the order of $10^{16} \mathrm{~cm}$ between the peaks of the $\mathrm{H} \alpha$ and [S II] emission, with the $\mathrm{H} \alpha$ occurring, as expected, closer to the ionizing source. The decline of the integrated $\mathrm{H} \alpha$ flux as the line of sight proceeds into the PDR also occurs in $\mathrm{Br} \gamma$. The lack of significant spatial offsets between these two hydrogen recombination lines implies that differential extinction from dust does not affect these profiles significantly. The fluoresced $\mathrm{H}_{2}$ emission is offset by $\sim 5 \times 10^{16} \mathrm{~cm}$ from the $\mathrm{Br} \gamma(1 \operatorname{arcsec}$ corresponds to $\left.3.4 \times 10^{16} \mathrm{~cm}\right)$. The profiles look similar to one-another even though we extracted them from different locations and projected angles relative to $\eta \mathrm{Car}$ and $\operatorname{Tr} 16$. When interpreting these spatial offsets one must keep in mind projection effects caused by the curvature of the globule. Ideally, numerical models would predict spatial emission 

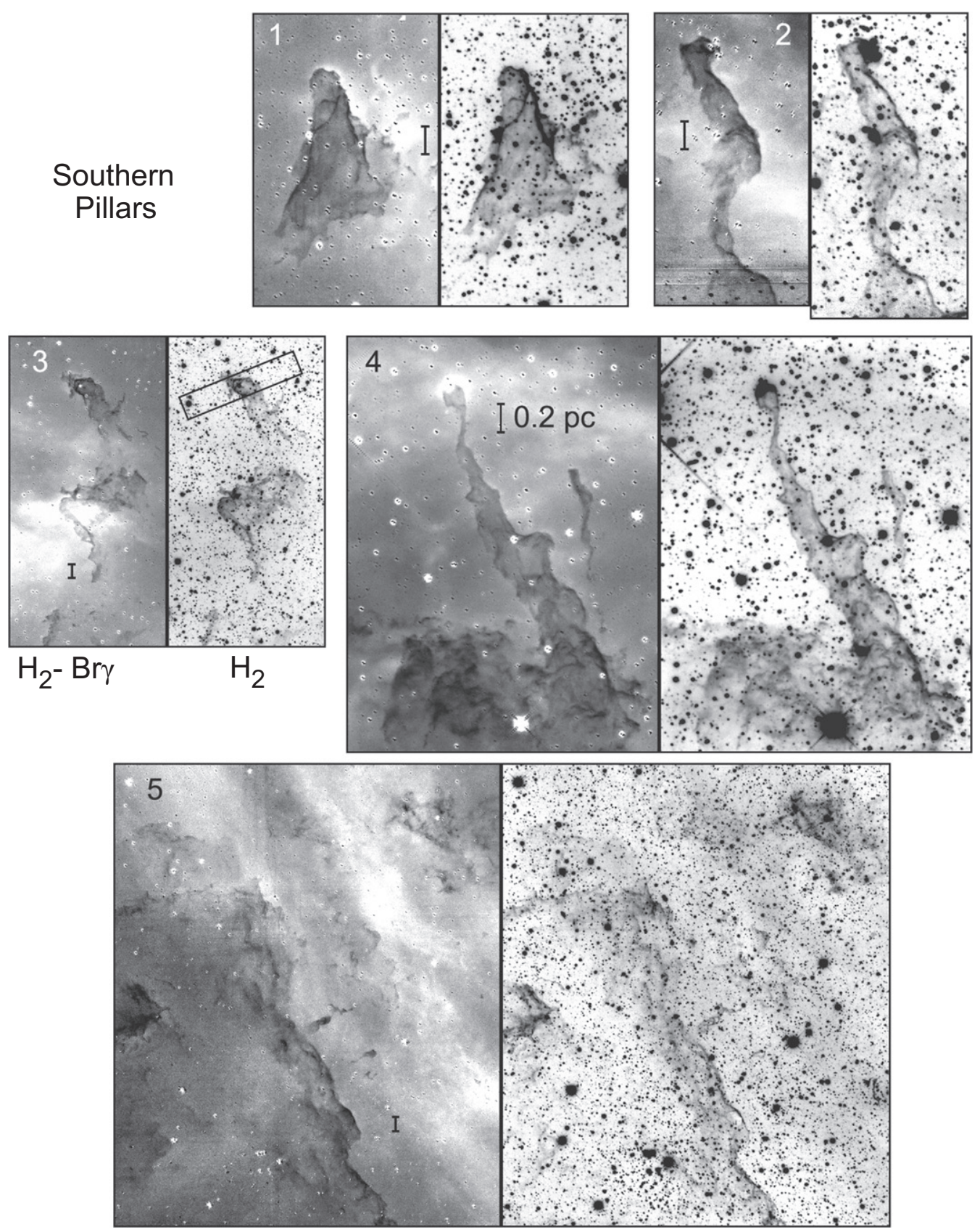

Figure 3. Southern Pillars of $\mathrm{H}_{2}$ emission in Carina. Numbers refer to the Areas 1-5 marked in Figure 2. Right: $\mathrm{H}_{2} 2.12 \mu$ m image, Left: difference image of $\mathrm{H}_{2}-\mathrm{Br} \gamma$, with $\mathrm{Br} \gamma$ emission relatively brighter where the image is white, and $\mathrm{H}_{2}$ relatively brighter where the image is black. The scale bar of $0.2 \mathrm{pc}$ corresponds to a distance of $2.3 \mathrm{kpc}$. The rectangle in Area 3 denotes the region covered in Figure 18.

profiles integrated along the line of sight to compare with these observations.

\section{JETS, OUTFLOWS, AND NEBULOUS OBJECTS}

Narrowband $\mathrm{H}_{2}$ imaging provides a view of the molecular outflows that are obscured at visual wavelengths and that may be unresolved with Spitzer's relatively coarse angular resolution at $\lambda>3 \mu \mathrm{m}$. Jets with $\mathrm{H}_{2}$ emission are usually still embedded in their natal clouds and shielded from the UV radiation that can dissociate molecules in exposed parts of the flows. In this section we first consider what the new images reveal about several previously known flows, go on to identify new molecular outflow candidates based on their $\mathrm{H}_{2}$ and $[\mathrm{S}$ II $]$ morphologies, and then discuss several peculiar nebulous objects. Half of the candidate molecular flows fall within the field of view imaged with $H S T$, whereas both new optical flows are in locations only imaged from the ground. Coordinates for the new candidate outflows are listed in Table 2.

\subsection{Known Outflows}

Smith et al. (2010a) detected 39 candidate and confirmed $\mathrm{HH}$ jets in the Carina Nebula, only one of which had been observed before in ground-based images (HH 666; Smith et al. 2004a). In hindsight, several of the $\mathrm{HH}$ jets discovered with HST are also visible in earlier ground-based images, but except for $\mathrm{HH} 666$, the jet-like morphologies of the HST 

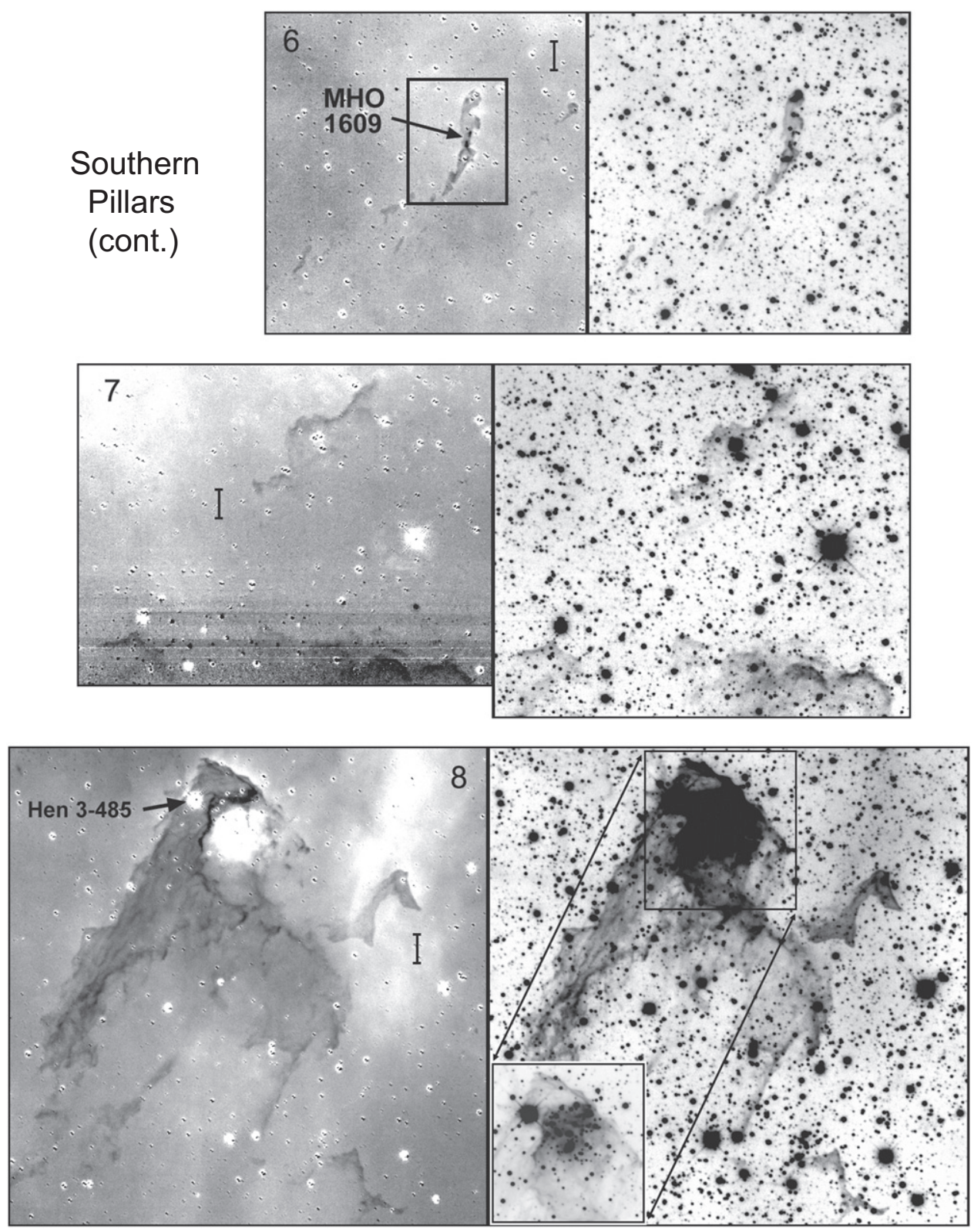

Figure 4. Same as Figure 3 for additional Southern Pillars of $\mathrm{H}_{2}$ emission in the Carina Nebula. Numbers refer to the Area 6-8 marked in Figure 2. The MHO 1609 region is expanded in Figure 27. The inset in Area 8 is the embedded cluster known as the Treasure Chest.

objects were unclear in the ground-based data. Thirty-five of the HST jets in Carina fall within the field of view imaged with NEWFIRM (the remaining four are in NGC 3324). While a few of these jets have $\mathrm{H}_{2}$ emission, most are too narrow or too faint to be discerned readily in the IR images. Nevertheless, the NEWFIRM images often provide valuable insights as to how irradiated pillars, globules, and the driving stars relate to the jets. In this section we discuss what the new images reveal about several of the most notable jets.

HH 666: HH 666 is a straight bipolar jet that emerges from the head of a dust pillar in the bright-rimmed globule G287.570.91 (Smith et al. 2004a; Area 3 in Figure 3; Figure 18). The pillar itself points toward $\eta$ Carinae. Optical imaging of this parsec-scale jet shows a chain of knots emitting in $\mathrm{H} \alpha$ and [S II] along the jet axis outside the globule, while narrow-band NIR $[\mathrm{Fe}$ II] images trace the flow into the obscured parts of the globule and back to the embedded driving source (Smith et al. 2004a; Reiter \& Smith 2013). The composite image in Figure 18 shows that knot $\mathrm{E}$ appears weakly in [O III], which could arise from a fast shock $\left(\gtrsim 100 \mathrm{~km} \mathrm{~s}^{-1}\right)$ into neutral gas, or from a slower shock into ionized gas.

NEWFIRM imaging uncovers some $\mathrm{H}_{2}$ emission inside the pillar, but none that is unambiguously associated with the jet. The $\mathrm{H}_{2}-\mathrm{Br} \gamma$ image in Figure 18 shows $\mathrm{H}_{2}$ emission along what appears to be the edges of a V-shaped cavity that originates at the source and opens to the northwest along the axis of the blueshifted jet (Smith et al. 2004a). This feature has a similar morphology to the IR reflection nebula seen in the IR images of HH 666 (Reiter \& Smith 2013), but it must have $\mathrm{H}_{2}$ emission because it persists strongly in the $\mathrm{H}_{2}-\mathrm{Br} \gamma$ image while reflected continuum would not. Additional $\mathrm{H}_{2}$ emission from the northern edge of the flow on both sides of the driving source may trace the molecular material excited in the walls of 

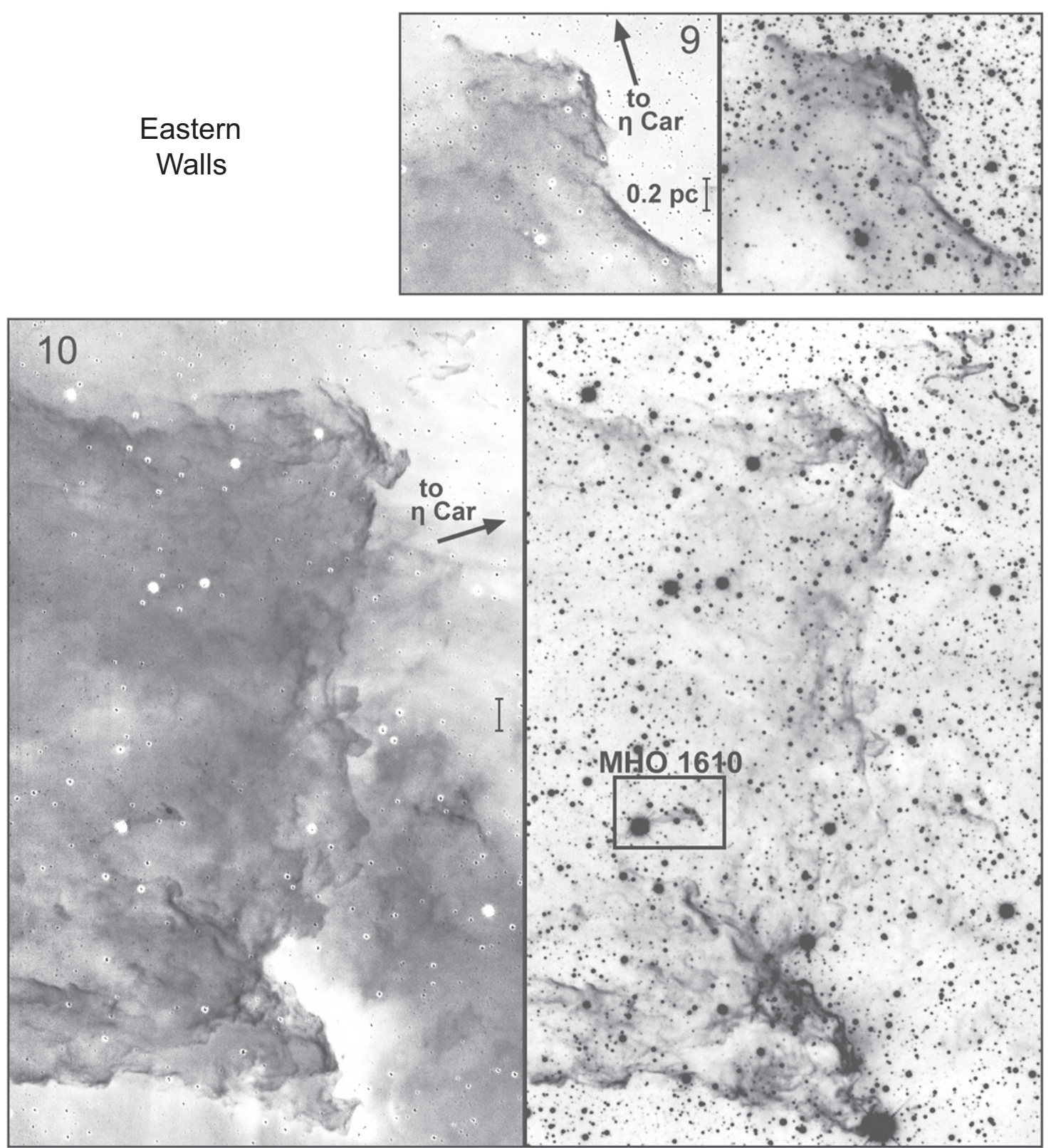

Figure 5. Same as Figure 3 but for Areas 9 and 10 of the Eastern Walls in Fig 2. The boxed region surrounding MHO 1610 is enlarged in Figure 28.

an outflow cavity cleared by the flow, although this is not certain given the complex filamentary background.

The driving source of the flow, HH666-IRS (located at 10:43:51.3 -59:55:21.2; Smith et al. 2004a), does not appear in the X-ray catalog of Broos et al. (2011). The object has a negative residual (white) in the $\mathrm{H}_{2}-\mathrm{Br} \gamma$ image in Figure 18. The ratio of the stellar fluxes in the $\mathrm{Br} \gamma$ and $\mathrm{H}_{2}$ narrowband images is enhanced by $\sim 15 \%$ relative to the median of stars in the field and to that expected for a flat continuum. However, the very red SED of HH666-IRS (Figure 9 of Smith et al. 2004a) will generate about $11 \%$ more flux in $\mathrm{Br} \gamma$ than in $\mathrm{H}_{2}$ owing to the $44 \mathrm{~nm}$ redder central wavelengths of the $\mathrm{Br} \gamma$ filter. If the remaining $4 \%$ excess were caused solely by line emission at $\operatorname{Br} \gamma$, it would imply an emission equivalent width of about $4 \%$ of the filter bandpass, or $\sim 9 \AA$, similar to what is observed for actively accreting classical $\mathrm{T}$ Tauri stars and Ae stars, both of which show correlations of $\mathrm{Br} \gamma$ line luminosity with disk accretion rates (Muzerolle et al. 1998; Donehew \& Brittain 2011). Hence, the observed $\mathrm{Br} / \mathrm{H}_{2}$ ratio for HH666-IRS is in line with that expected given the extremely red observed SED of the source, and $\operatorname{Br} \gamma$ emission could explain the remaining small excess. However this inference will need to be confirmed spectroscopically.

$H H$ 900: a dark globule (visible in Area 11 in Figure 6, and in the $[\mathrm{O}$ III] image in Figure 19) has a narrow $(\sim 0 \prime 2)$ rope-like tail that extends to the northeast that is visible only at $H S T$ resolution (figure 4 of Smith et al. 2010a). The globule lies within a wider $\sim 2^{\prime \prime}$ streak of $\mathrm{H} \alpha$ emission that extends along the same direction on either side of the globule and points toward of a pair of oppositely directed bow shocks. Smith et al. (2010a) identified the $\mathrm{H} \alpha$ streak as a jet associated with the globule, but also noted the presence of a star near the western edge of the streak that may drive a separate microjet. 

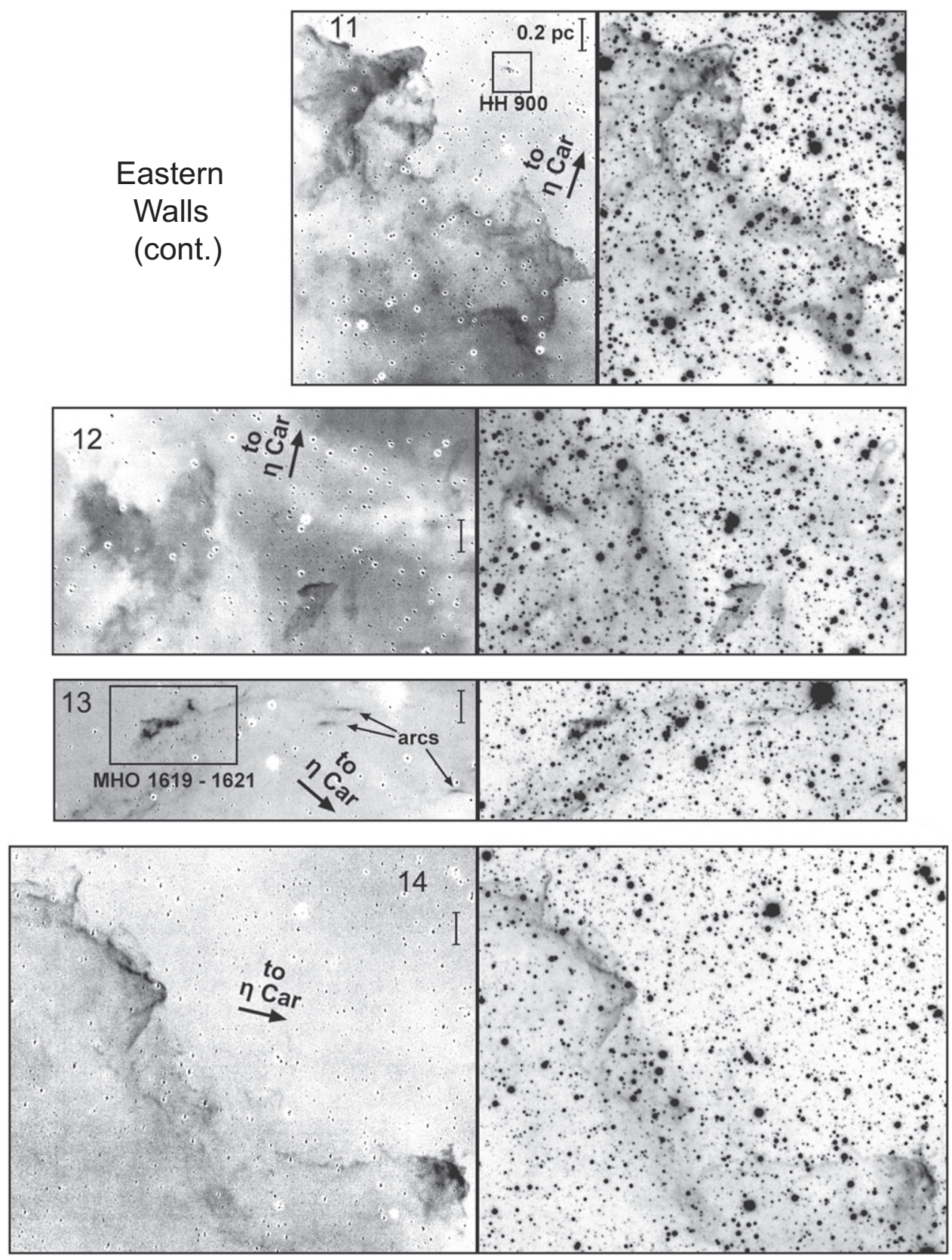

Figure 6. Same as Figure 3 but for Areas 11 through 14 of the Eastern Walls in Figure 2. The small boxed region in Area 11 is HH 900, reproduced in Figure 19, and MHO 1619-MHO 1621 in Area 13 is expanded in Figure 31. The direction to $\eta$ Car is shown.

Of the three brightest stars near the globule (marked with "+" in Figure 19), only the one labeled IRS (also known as 2MASS J10451881-5944238 or PCYC 838; Povich et al. 2011a) at the western end of the $\mathrm{H} \alpha$ streak appears unusual in that it is significantly brighter in $\mathrm{Br} \gamma$ than it is in $\mathrm{H}_{2}$, indicative of a very red source and/or one with strong $\mathrm{Br} \gamma$ emission (as described for HH666 IRS above). The SED of this source based on 2MASS and Spitzer photometry exhibits a strong mid-IR excess (Povich et al. 2011a). The best fit of the grid of young stellar object (YSO) models of Robitaille et al. (2006) obtained with the on-line SED fitting routine (Robitaille et al. 2007) occurs for a nearly edge-on disk around a $2.3 M_{\odot}$ star of age $8.9 \times 10^{4} \mathrm{yr}$, with a total luminosity of $45 L_{\odot}$ and a disk accretion rate of $1.1 \times 10^{-9} M_{\odot} \mathrm{yr}^{-1}$. However, uncertainties on such model fits can be large (Offner et al. 2012); for example, Povich et al. (2011a) report log $L=2.1 \pm 1.4 L_{\odot}$ and $M=2.5 \pm 1.2 M_{\odot}$ for this source.

The $\mathrm{H}_{2}$ emission in Figure 19 is brightest on the northern side of the dark globule, and extends along the $\mathrm{H} \alpha$ streak both to the northeast and to the southwest. The $\mathrm{H}_{2}$ is offset to the south of the $\mathrm{H} \alpha$ as would occur if the globule were irradiated by sources in $\operatorname{Tr} 16$ to the north. However, further interpretation of this unusual object is difficult to do from ground-based images because many of the key features such as the dark ropelike tail are unresolved, and two potential exciting sources are superposed upon the $\mathrm{H}_{2}$, [S II], and $\mathrm{H} \alpha$ nebular structures. New $H S T$ images and spectroscopy of the object will appear in a future work that will investigate this object more fully (M. Reiter et al. 2015, in preparation). 


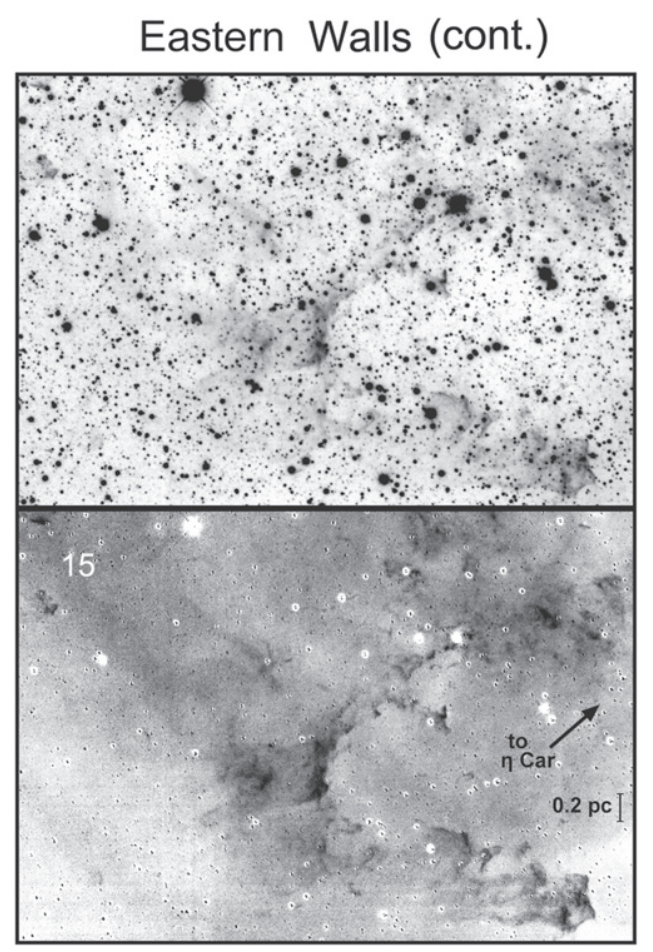

Figure 7. Same as Figure 3 but for Area 15 of the Eastern Walls in Figure 2. The $\mathrm{H}_{2}$ image is at top with the difference image below it.

HH 901: HH 901 is a jet that emerges from the head of a pillar that is irradiated by $\mathrm{O}$ stars in $\operatorname{Tr} 14$ and $\operatorname{Tr} 16$ (Smith et al. 2010a) Area 29 of Figure 10). Our images of this source in Figure 20 show the typical structure of an irradiated pillar, with $\mathrm{H}_{2}$ emission surrounded by limb-brightened $\mathrm{H} \alpha$ and $\mathrm{Br} \gamma$. A diffuse arc which is bright in $\mathrm{H} \alpha$ and [O $\mathrm{OII}]$ cuts across the middle of the pillar. This feature lies along the extension of the bow shock, although the high resolution HST images suggest the bow shock and the arc may be unrelated to one another.

The $\mathrm{H}_{2}-\mathrm{Br} \gamma$ panel in the upper right of Figure 20 shows that the $\mathrm{HH} 901$ jet emits in $\mathrm{H}_{2}$ as it emerges from the pillar. An $\mathrm{H}_{2}$ knot marks the location where the jet emerges from the west side of the pillar, while it is possible to trace the jet in $\mathrm{H}_{2}$ for about $0.02 \mathrm{pc}$ to the east of the pillar. If $\mathrm{H}_{2}$ emission came only from the irradiated edge of the pillar, we would expect the emission to trace the ionization front and peak at the apex of the arc facing Trumpler 14. Instead, the $\mathrm{H}_{2}$ emission is bright at the edge of the globule on the west side, and extends along the optical jet to the east, arguing that $\mathrm{H}_{2}$ originates in the outflow itself.

A continuum source labeled IRS in Figure 20 appears along the jet near the eastern edge of the pillar in the K-band and $\mathrm{Br} \gamma$ images at 10:44:03.58 -59:31:01 (2000), and is also visible in the I-band image. However, the stellar density is high enough in the NIR that chance superpositions are a concern, and in HST images (Reiter \& Smith 2013) the source lies above the jet axis and just outside the edge of the pillar. The object was not listed in the catalog of X-ray-selected members of Carina (Broos et al. 2011).

HH 902: situated just above HH 901, the HH 902 jet is similar in that it originates from the tip of an irradiated pillar, though in this case the pillar is much wider (Smith et al. 2010a; Area 29 in Figures 10;21). A jet appears to the west of the pillar in the $\mathrm{H} \alpha$ images in Figure 21, and also somewhat less- distinctly in $\operatorname{Br} \gamma$. The situation to the east is more complex. The $H S T$ image reveals a complex morphological structure in $\mathrm{H} \alpha$ where the projection of the jet intersects the base of the pillar that contains HH 901. Reiter \& Smith (2014) show that the eastern and western knots both move away from the pillar along the axis of the jet. This region has a strong continuum component in our K-band images.

The $\mathrm{H}_{2}$ emission near $\mathrm{HH} 902$ is rather unremarkable, and simply follows the outline of the pillar. A small nebulous knot in the $\mathrm{H}_{2}$ image along the jet labeled "cont" in Figure 21 appears to be a continuum source, since its flux ratio of $\mathrm{H}_{2} / \mathrm{K}$ is similar to that of field stars. The object also appears in $H S T$ images in [Fe II] (Reiter \& Smith 2013), and is likely to be unrelated to $\mathrm{HH} 902$ because knots on either side of it have proper motions in the same direction (Reiter \& Smith 2014). A star in the K-band image exists near the head of the pillar at coordinate J2000 10:44:01.61-59:30:29 (Ohlendorf et al. 2012). However, the star field here is fairly dense, and there are no other indications (e.g., very red colors, reflectedlight cavities, X-ray emission) that this source is driving the jet. Its position is about 2 arcsec away from where Smith et al. (2010a) suspected the driving source should be located based on morphologies in their HST images, and is also about 2 arcsec north of the axis defined by the $\mathrm{H} \alpha$ proper motions measured by Reiter \& Smith (2014). As for the other irradiated globules and pillars, the host pillar for $\mathrm{HH} 902$ shows a spatial offset between $\mathrm{H}_{2}$ and $\mathrm{Br} \gamma$ in its difference image (Figures 10 and 21) in the sense that the $\mathrm{Br} \gamma$ forms a sheath on the outside of the $\mathrm{H}_{2}$ emission from the pillar.

HH 1066: the newly confirmed jet $\mathrm{HH} 1066$ (formerly $\mathrm{HH}$ c1 ) is driven from the head of a bright C-shaped proplyd (Reiter \& Smith 2013). The proplyd is superposed upon a larger pillar in the background that itself has small-scale structure suggestive of at least three more pillars (Smith et al. 2010a; Area 29 of Figure 10). The illumination source is to the south, in the general direction of $\operatorname{Tr} 14$. The composite images in Figure 22 show the proplyd and the large pillar well in $\mathrm{H} \alpha$, $\left[\mathrm{S}_{\mathrm{II}}\right]$, and even [O III] but the jet is only visible weakly in $\mathrm{H} \alpha$ and $[\mathrm{S}$ II $]$ in our ground-based images.

The $\mathrm{H}_{2}-\mathrm{Br} \gamma$ difference image reveals the typical outline of inner $\mathrm{H}_{2}$ emission enclosed by a shell of $\mathrm{Br} \gamma$. However, toward the southwestern edge of the large pillar we find a spot of bright $\mathrm{H}_{2}$ that appears to separate more from the corresponding $\mathrm{Br} \gamma$ emission to its south. The HST images hint that several interfaces converge at this point which may account for the additional $\mathrm{H}_{2}$. It is also possible that a shocked knot exists at this location, but high-resolution images of this region with $H S T$ or ground-based AO will be needed to interpret the structure at this location. The exciting star located near the apex of the proplyd is PCYC 429, which has a NIR excess and is an X-ray source (Broos et al. 2011; Povich et al. 2011a).

HH c-9: Smith et al. (2010a) drew attention to a parabolic arc in the $\operatorname{Tr} 14$ region that was bright in $\mathrm{H} \alpha$ and [O III], and present but fainter in $[\mathrm{S}$ II] . It is located 1 arcmin due east of the cluster in Area 30 (Figures 2 and 10). Our Br $\gamma$ images show a similar morphology to the $\mathrm{H} \alpha$ and [O III] images in Figure 26. The object has no $\mathrm{H}_{2}$ emission. The arc is a highly ionized shell of some sort, probably either a bow shock or the edge of a stellar wind bubble irradiated by stars in Trumpler 14. If it is a bow shock there is no obvious source for the flow in either the $\mathrm{H}_{2}$ or $\mathrm{Br} \gamma$ images. Proper motion measurements will be the best way to determine the nature of this object. 

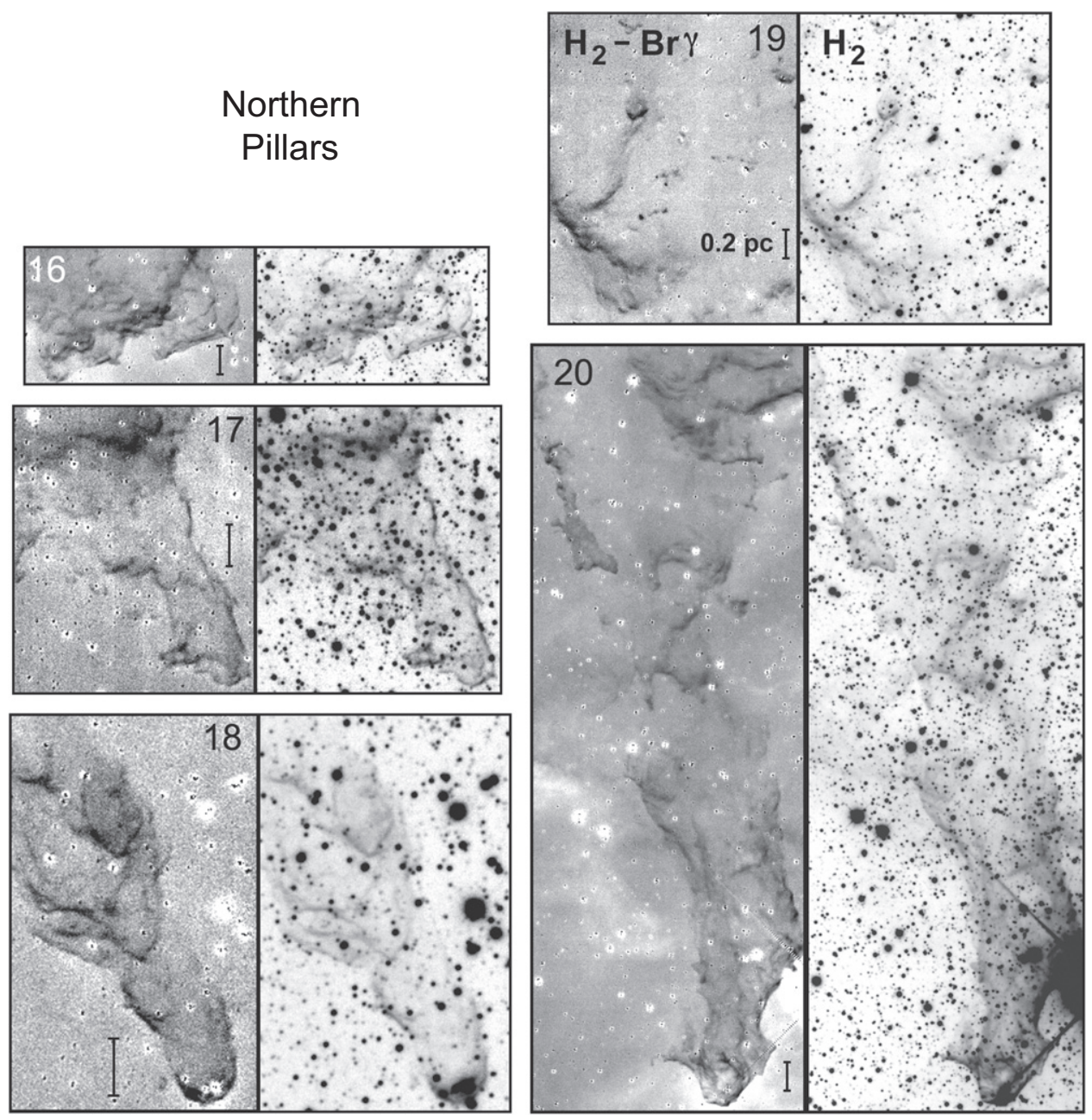

Figure 8. Same as Figure 3 but for Areas 16 through 20 of the Northern Pillars in Figure 2. Figure 32 presents an expanded version of the MHO objects in Area 19.

HH 903 and HH c-10: The Smith et al. (2010a) HST ACS $\mathrm{H} \alpha$ images of the southern pillar G287.88-0.93 show two distinct outflows. HH 903 originates from the center of the pillar and drives an optical jet to the west, with bow shocks that extend about an arcminute to both the east and west of the

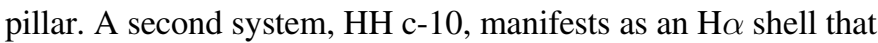
extends about 10 arcsec to the southwest of a small cluster of stars located at the head of the pillar. Neither of these flows are easy to see in our NEWFIRM images (Area 2 of Figure 3), though it is possible to see faint traces of the $H S T$ flows in our $\mathrm{Br} \gamma$ images. $\mathrm{H}_{2}$ emission is not visible in the $\mathrm{HH} 903$ jet and bow shocks or in the HH c-10 shell. However, at the location of the source of the HH 903 jet the pillar has several curved arcs like we observe at the heads of other pillars. This morphology suggests the pillar is either two pillars observed in projection, or that the pillar has a "shoulder" where the driving source of the $\mathrm{HH} 903$ jet lies.

$H H 1008$ and $H H c$-2: The pillar G287.73-0.92 (Area 4 of Figure 3) is one of the most remarkable of its kind in the sky. HH 1008 is situated near the base of the pillar on the eastern side, and appears as a southward-moving bow shock in HST $\mathrm{H} \alpha$ images (Smith et al. 2010a). This feature is visible in our $\mathrm{Br} \gamma$ images as well, albeit with less contrast owing to groundbased seeing. The candidate $\mathrm{HH}$ object $\mathrm{c}-2$ located at the head of the pillar is too small to be detected from the ground. Neither object has $\mathrm{H}_{2}$ emission.

\subsection{New Outflows}

We identified new outflow candidates in the Carina Nebula region via their morphology in our narrowband NEWFIRM and MOSAIC images. Optical outflow candidates all have bright $[\mathrm{S}$ II] emission, typical of $\mathrm{HH}$ objects. Several molecular jets and shocks appear in the $\mathrm{H}_{2}$ images alone, but most are easier to see in the $\mathrm{H}_{2}-\mathrm{Br} \gamma$ difference images because continuum is present in similar amounts in both images and is largely removed in the subtraction. However, $\mathrm{H}_{2}$ emission along PDRs (Section 3.1) can be difficult to distinguish from shocked molecular flows in Carina. Following Davis et al. (2010), we designate objects that have clumpy or jet-like $\mathrm{H}_{2}$ emission and do not define obvious PDR boundaries with the acronymn MHO (molecular hydrogen object). Section 4.3 summarizes extended $\mathrm{H}_{2}$ objects whose true nature remains uncertain from morphology alone.

\subsubsection{New Optically Identified Outflows and Candidates}

HH 1123: HH 1123 consists of a group of four bright $\mathrm{H} \alpha$ and $\left[\mathrm{S}_{\mathrm{II}}\right]$ knots arranged in a cross-shape (Figure 23). The knots have the typical clumpy morphology of $\mathrm{HH}$ objects, and are not visible in $\left[\mathrm{O}\right.$ III], $\mathrm{Br} \gamma$ or in $\mathrm{H}_{2}$. A relatively faint star lies between knots $\mathrm{A}$ and $\mathrm{C}$ in the IR images, but this source is not clearly associated with the emission knots. There is no indication of a jet, although a faint arc that extends from knot 


\section{Northern
Pillars
(cont.)}
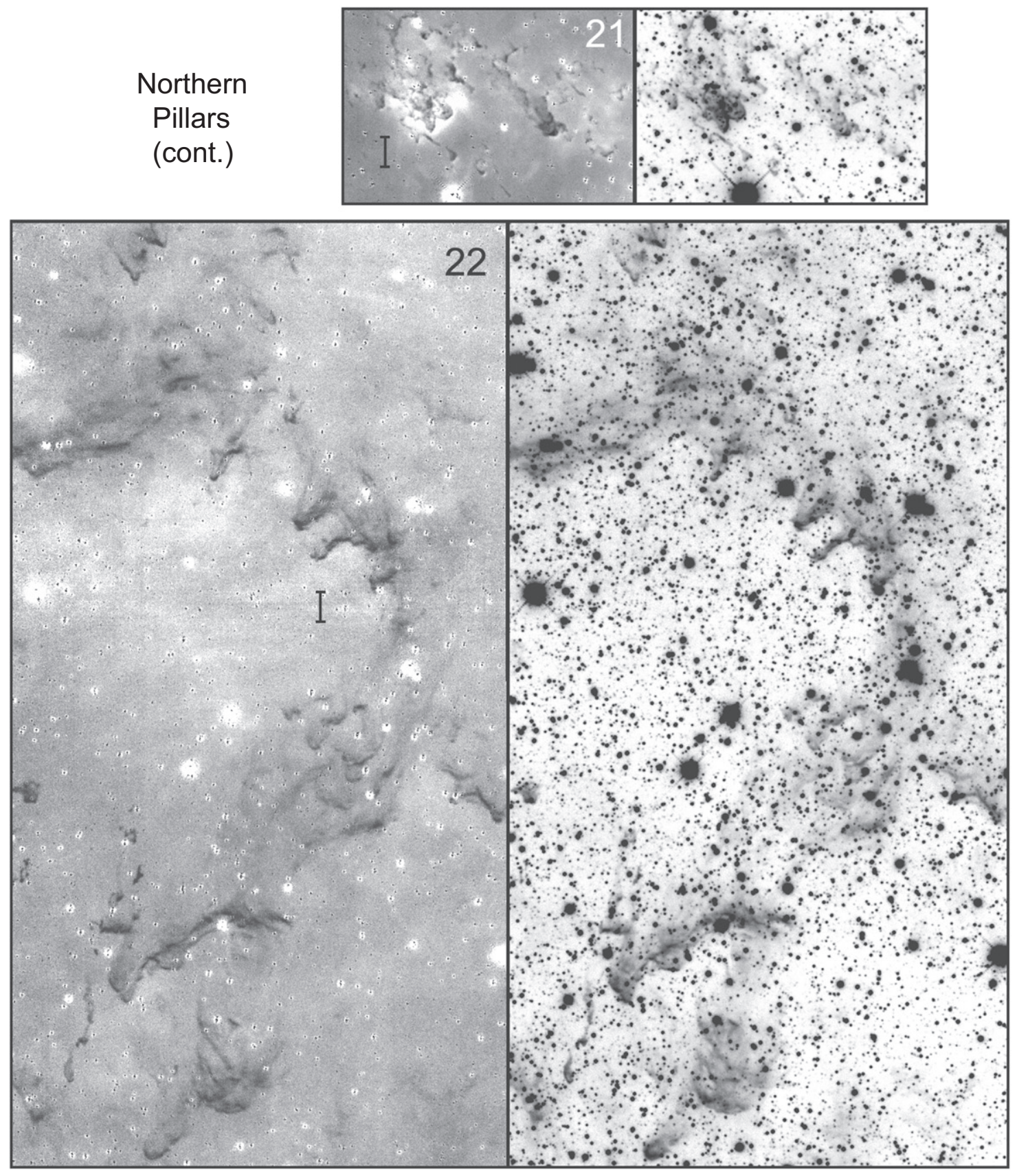

Figure 9. Same as Figure 3 but for Areas 21 and 22 of the Northern Pillars in Figure 2.

A to the east of knot $\mathrm{C}$ may mark the edge of a cavity. HH 1123 is situated along the northern extension of the Southwestern Loop. The Southwestern Loop is a region that hosts many filamentary structures and knots that appear to result from impacts of winds upon a large, slowly expanding shell (Figure 34), and it is possible that $\mathrm{HH} 1123$ has a similar origin.

$\mathrm{HH}$ 1124: images of the head of the irradiated pillar in Area 18 of Figure 8 in $\mathrm{H} \alpha,[\mathrm{S} \mathrm{II}], I$-band, and $\mathrm{H}_{2}$ are shown in Figure 24. Three optical emission-line knots (A-C) lie to the west of the pillar, while one knot (D) and a bow-shaped arc lie to the east. If we attribute all knots to a single source, then the pattern is consistent with a jet that emerges at $\mathrm{PA} \sim 53^{\circ}$ and is bent to the north by winds from the same source or sources that shape the pillar. The $\mathrm{H}_{2}$ images show weak extensions along the axis of the putative jet that are not continuum because they are also visible in the $\mathrm{H}_{2}-\mathrm{Br} \gamma$ images in Figure 8. However, this $\mathrm{H}_{2}$ "jet" marked in Figure 24 could also simply define another irradiated surface along the pillar. The axis of the outflow also has extended emission in IRAC Band 2 images, including an extended green object (EGO) identified from a Band 2-Band 1 difference image.

There are several IR sources located near the head of the main pillar, so confusion between multiple outflows is always a concern. Two bright NIR sources positioned near the center of the pillar, PCYC 884 and PCYC 889, are YSOs as they emit strongly in the Spitzer bandpasses (Povich et al. 2011a; Figure 24) PCYC 884 lies at the base of an arc-shaped feature in the I-bandpass and is the only IR source in the region associated with such a feature. This arc has a morphology and 

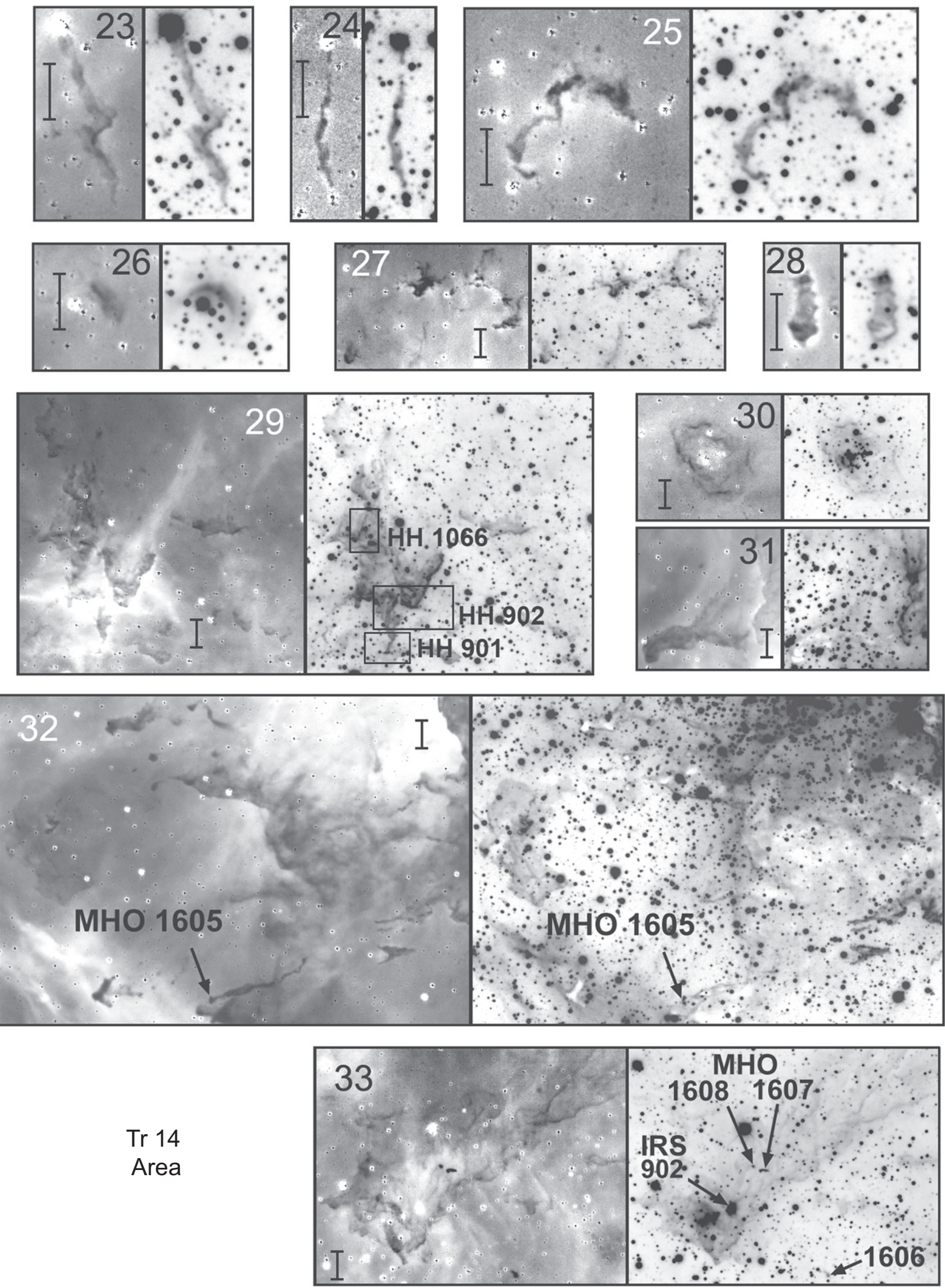

Figure 10. Same as Figure 3 but for Areas 23 through 33 of the Trumpler 14 region in Figure 2. Boxes around HH 901, HH 902, and HH 1066 are expanded in Figures 20-22, respectively. The MHO objects in Areas 32 and 33 are discussed in the text. 

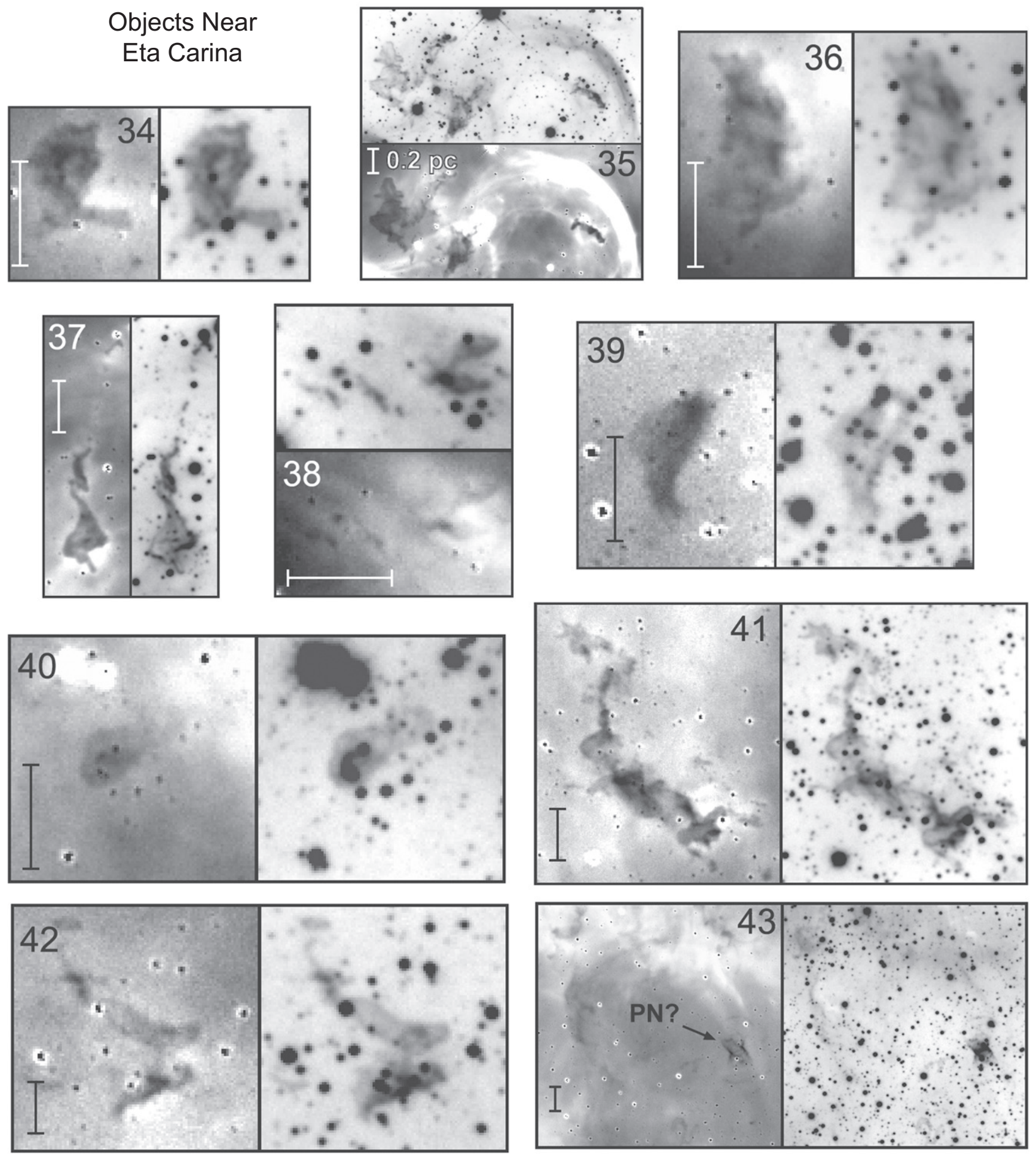

Figure 11. Same as Figure 3 but for Areas 34 through 43 in the vicinity of $\eta$ Car in Figure 2. The nebulous object on the right side of Area 43 is a candidate planetary nebula.

orientation consistent with it defining the edge of an evacuated cavity driven by the outflow that produces knots $\mathrm{A}, \mathrm{B}$, and $\mathrm{C}$. The source has a flat SED in the IRAC bands. Situated just to the west of PCYC 884, PCYC 889 is fainter in the NIR, but becomes a magnitude brighter than PCYC 884 at $24 \mu \mathrm{m}$ (Povich et al. 2011a).

A region outlined by a box in Figure 24 and located $\sim 0.1 \mathrm{pc}$ to the west of a NIR source (IRS 1 in the figure) has several faint knots and extended emission in the $\mathrm{H} \alpha$, [S II] and $\mathrm{H}_{2}$ images. The knots are not necessarily associated with IRS 1; in fact, the $\mathrm{H}_{2}-\mathrm{Br} \gamma$ difference image in Area 18 of Figure 8 has an arc-shape, so this region is most likely another irradiated pillar. The object labeled IRS 2 is another red object in the direction of the globule. It lies along the axis of the main outflow and appears to be a subarcsecond binary with a $\mathrm{PA} \sim 105^{\circ}$. Finally, a bright star just outside the edge of the 


\section{Southwestern Pillars and Walls}


Figure 12. Same as Figure 3 but for Areas 44 through 50 of the Southwestern Pillars and Walls in Figure 2 . The $\mathrm{H}_{2}$ images are at the top or right in each panel, with the $\mathrm{H}_{2}-\mathrm{Br} \gamma$ images at bottom or left.

pillar $\sim 0.06 \mathrm{pc}$ south of PCYC 884 is probably a foreground object, as it is bright in both the optical and IR images and does not have any obvious influence on the morphology of the pillar.

HH 1125: $\mathrm{HH} 1125$ is a thin, long, filamentary [S II] emission-line object located near HH 666 in the region of the southern pillars to the west of the $\mathrm{H}_{2}$ structures shown in Area 3 of Figure 3. The morphology of HH 1125 resembles other filamentary $\left[\mathrm{S}_{\mathrm{II}}\right]$ structures that trace the remains of an outflow lobe from an unseen driving source, such as HH 400 in Orion (Bally et al. 2001). The brightest feature in this object (marked "A" in Figure 25) has a morphology similar to that of a bow shock in a jet that propagates at position angle $280^{\circ}$. HH 1125 is invisible in $\mathrm{H} \alpha$ and $[\mathrm{O} \mathrm{III}]$, and also has no IR counterparts in $\mathrm{H}_{2}$ or $\mathrm{Br} \gamma$, so it is unlikely to mark the edge of a dark cloud. The projected distance of the most distant observed knot $\mathrm{B}$ from the edge of the nebulous region is $3.7 \mathrm{pc}$, so HH 1125 would represent a large-scale outflow if it traces a jet. While any number of the bright stars in this densely populated region could be the exciting source for a jet, none of them particularly stand out as an obvious driving source. Hence, without radial velocity and proper motion 
Northwestern Pillars, Walls, and Jets
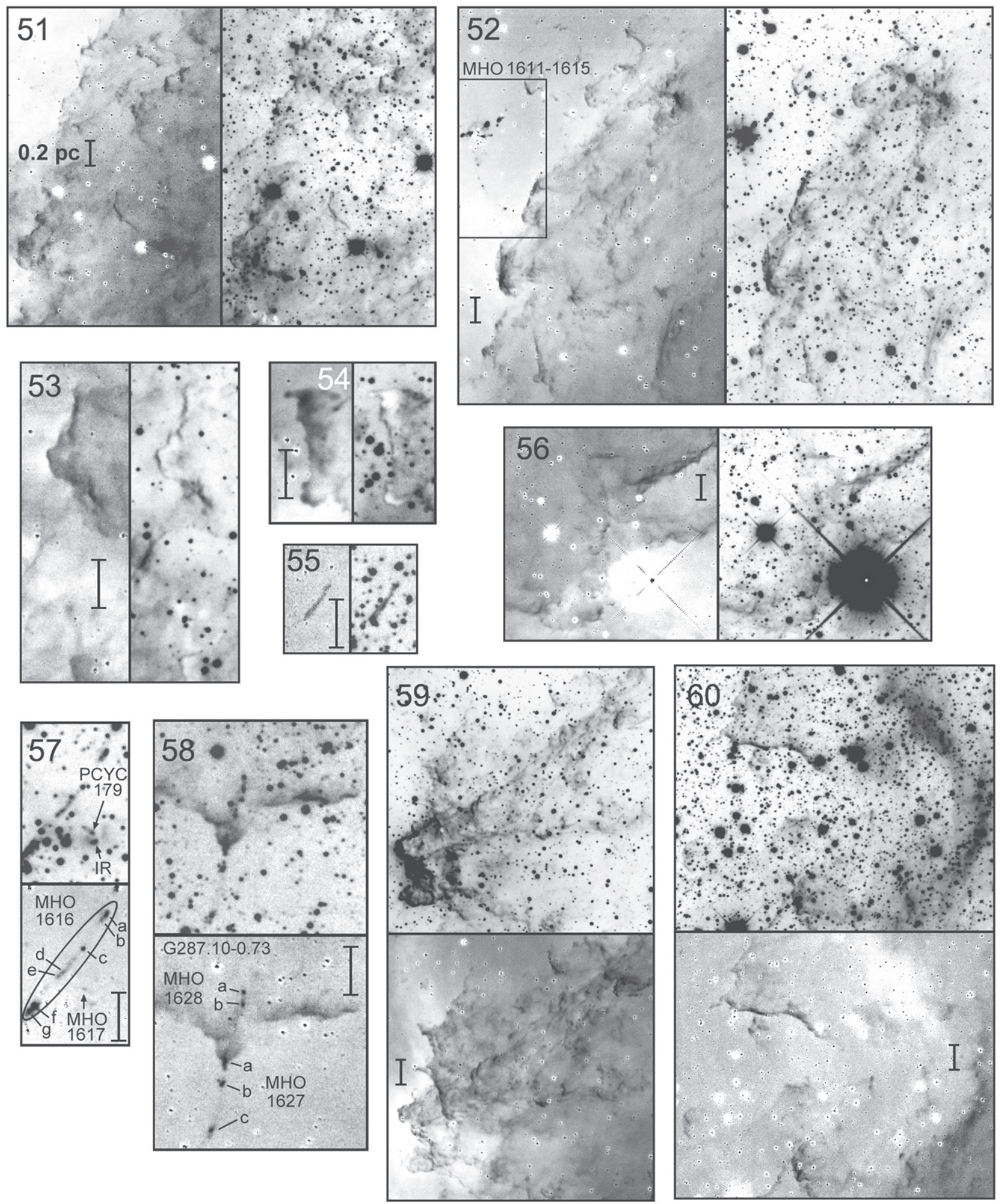

Figure 13. Same as Figure 3 but for Areas 51 through 60 of the Northwestern Pillars, Walls and Jets in Figure 2. The $\mathrm{H}_{2}$ images are at the top or right in each panel, with the $\mathrm{H}_{2}-\mathrm{Br} \gamma$ images at bottom or left. The region marked in Area 52 is expanded in Figure 29.

information we cannot rule out the possibility that HH 1125 represents the edge of a shell driven by one of the many massive stars in Carina.
HHc-21: Figure 32 shows a small but distinct optical knot located about $1.5 \operatorname{arcsec}$ at $\mathrm{PA}=200^{\circ}$ away from a star. The knot, also faintly visible in $\left[\mathrm{S}_{\mathrm{II}}\right]$, but not in $\left[\mathrm{O}_{\mathrm{III}}\right], \mathrm{H}_{2}$, or $\mathrm{Br} \gamma$, is 

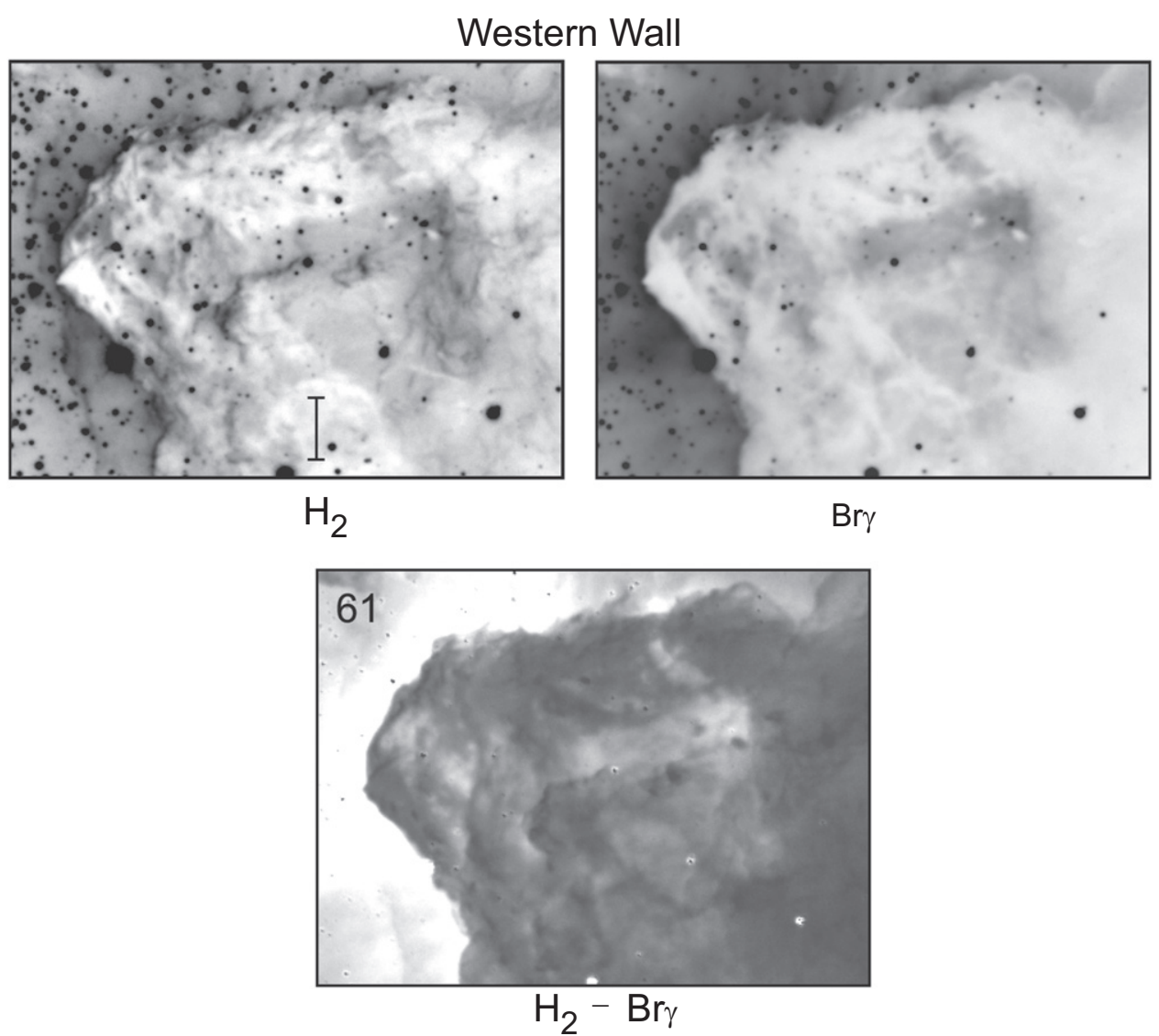

Figure 14. Same as Figure 3 but for Area 61 (G287.38-0.62), the Western Wall in Figure 2. The $\mathrm{H}_{2}$ and $\mathrm{Br} \gamma$ images are at the top, with the $\mathrm{H}_{2}-\mathrm{Br} \gamma$ images at bottom.

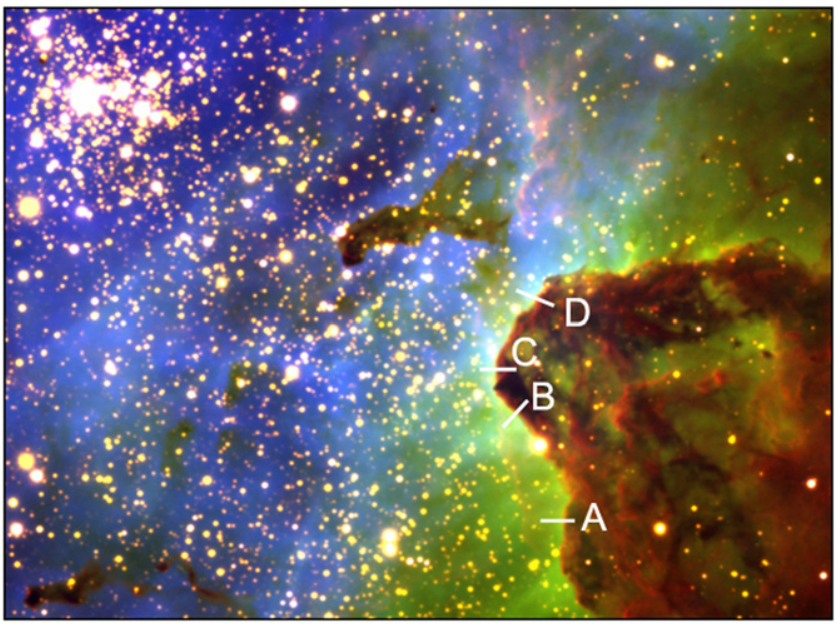

Figure 15. Color composite of the Western Wall in Figure 14, with $\mathrm{H}_{2}$ in red, $\mathrm{Br} \gamma$ in green, and $[\mathrm{O}$ III] $5007 \AA$ in blue. Positions A-D mark the four slices along the PDR interface used in Figure 16 to demonstrate positional offsets in the emission line fluxes.

a candidate $\mathrm{HH}$ object we label HHc-21. The star is not listed as a member of Carina by Povich et al. (2011a). The system lies to the north of the $\mathrm{H} \alpha$ arc R2 in the region of MHO 1622 MHO 1626.

\subsubsection{Candidate Outflows Identified in Narrowband $\mathrm{H}_{2}$ Images}

We have searched our images for evidence of shocked $\mathrm{H}_{2}$ in outflows. Such objects typically appear as distinct knots rather than extended $\mathrm{H}_{2}$ that defines a PDR front at the edge of a globule, wall or pillar. Morphologies of the PDRs in Carina can be quite complex, however, and it is not always immediately obvious from images alone which objects are shocked flows and which are PDRs. For this reason MHO objects in the Carina Nebula are best treated as candidate outflows pending verification by proper motions, though most will likely turn out to be outflows. A few of these were discovered independently by Preibisch et al. (2011b), and we discuss those objects together with the new sources in this section.

MHO 1605: MHO 1605 has the morphology of a barely resolved bipolar outflow emerging from a small pillar embedded in the dark dust lane $\sim 4^{\prime}$ south of Trumpler 14 (Preibisch et al. 2011b) Area 32 of Figure 10). The object consists of two bright $\mathrm{H}_{2}$ knots located just outside the pillar, along $\mathrm{PA} \approx 3^{\circ}$. The pillar is well-defined as an extinction feature in the $\mathrm{Br} \gamma, \mathrm{H} \alpha$, [S II] , and [O III] images. MHO 1605 is difficult to discern in the optical images, although there is some diffuse $\left[\mathrm{S}\right.$ II] emission coincident with the $\mathrm{H}_{2}$ knots. Emission in IRAC bands 1-3 is evident near the pillar head and is elongated along a north-south axis (not consistent with a point source), but the feature is too small (only $\sim 4^{\prime \prime}$ long in our $\mathrm{H}_{2}$ image) to be clearly resolved with Spitzer.

Cr 232/N4 Area, MHO 1606-1608: Preibisch et al. (2011b) and Tapia et al. (2011) discussed the cluster $\mathrm{Cr} 232$ that is associated with the extended $\mathrm{H}_{2}$ emission in Area 33 of Figure 10. The bright $K$-band source labeled IRS in Figure 10 is a massive star embedded within a nearly edge-on circumstellar disk that Preibisch et al. (2011d) refer to as the “disk object" (also known as source \#902, Tapia et al. 2011). A 


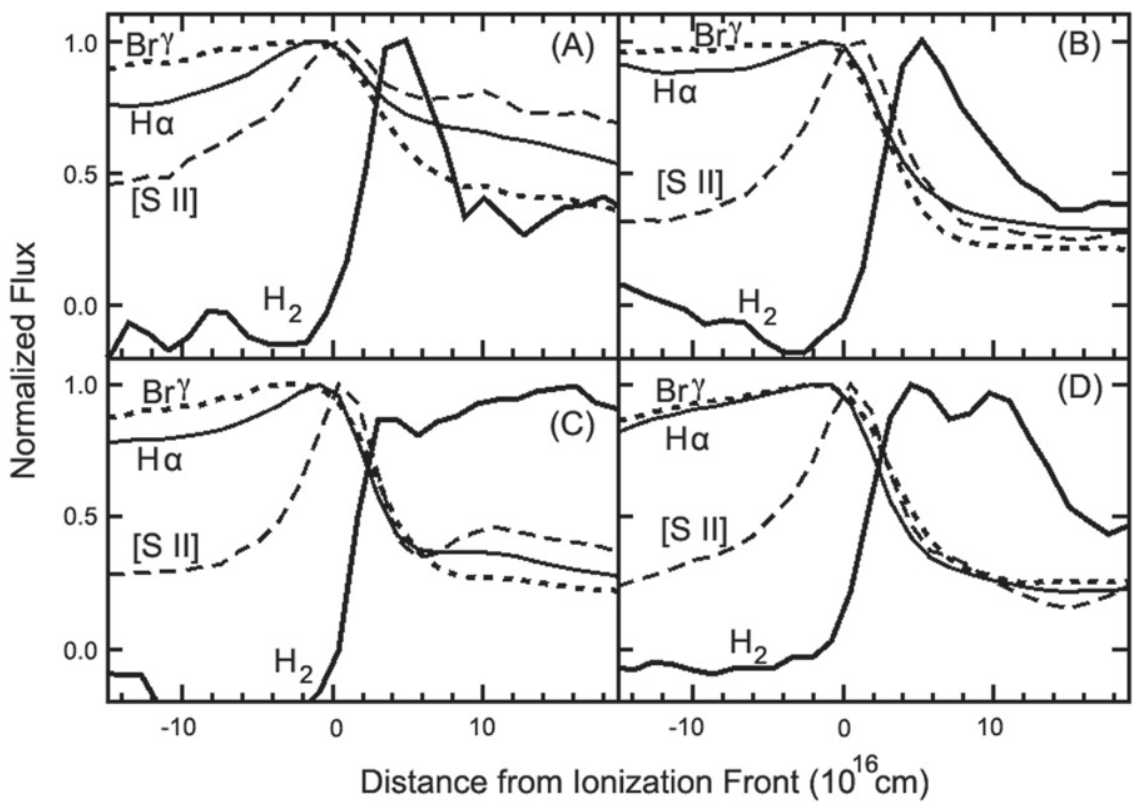

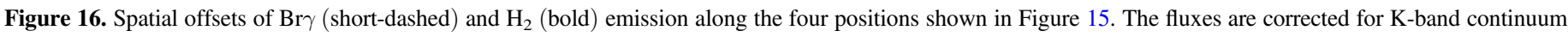
as described in the text. Note the distinct offset between $\mathrm{H}_{2}$ and $\mathrm{Br} \gamma$.

separate foreground X-ray source, PCYC 556, is located about 2 arcsec to the southeast of the NIR peak, and appears in our NIR images and those of Preibisch et al. (2011d) as a distinct point source. Figure 10 uncovers over a dozen localized bright regions of $\mathrm{H}_{2}$ emission in this region. Most of these follow arcs that define PDR boundaries in the dark cloud that houses the cluster, though several may turn out to result from shocks in outflows. Three bright $\mathrm{H}_{2}$ features particularly stand out in the $\mathrm{H}_{2}-\mathrm{Br} \gamma$ image as having a knotty morphology typical of shocked gas. The brightest of these, MHO 1607, consists of three distinct knots aligned approximately east to west over an extent of about 5 arcsec. A few arcseconds to the northeast, MHO 1608 is more point-like, with a slight elongation along PA $45^{\circ}$. Both these $\mathrm{MHO}$ objects are prominent in the $\mathrm{H}_{2}$ image but are not present in $\mathrm{Br} \gamma$. The $\mathrm{H}_{2}-\mathrm{Br} \gamma$ image reveals two faint arcs that trail to the southeast of MHO 1608 and may outline the edges of an irradiated pillar. Neither the optical nor the IR images indicate any obvious driving source associated with these two objects, though of course there are many possibilities within the cluster, which has 72 members according to Tapia et al. (2011). The IRAC Band 2-Band 1 image shows diffuse emission in the area, with a modestly bright feature located between MHO 1607 and 1608. A third object, MHO 1606, is an isolated, bright, comma-shaped $\mathrm{H}_{2}$ knot located away from the cloud boundary $\sim 80$ arcsec to the southwest of MHO 1607 and MHO 1608. This object also has weak $[\mathrm{S}$ II] emission.

MHO 1609: the detached pillar shown in Area 6 of Figure 4 and in Figure 27 is surrounded by several candidate $\mathrm{HH}$ objects (HH c-4 through HH c-8) identified by Smith et al. (2010a), but none of these are visible in our NEWFIRM images. However, our $\mathrm{H}_{2}$ images uncover two extended spots of bright emission near the middle of the irradiated pillar. These spots lie just inside the $\mathrm{H} \alpha$ and $\left[\mathrm{S}_{\mathrm{II}}\right]$ emission at the surface of the evaporating pillar. A $K$-band point source lies just west of the $\mathrm{H}_{2}$ knots, but is offset from the probable flow axis enough that it is unlikely to be the driving source. It is unclear if the two elongated features are related as they are bright in a relatively narrow portion of the pillar, and at this resolution, are consistent with emission from the irradiated pillar edge. An alternative interpretation is that bright $\mathrm{H}_{2}$ emission comes from photoevaporation of the denser clumps within a more diffuse pillar. None of the $\mathrm{H}_{2}$ features in the pillar appear to be related to any of the candidate $\mathrm{HH}$ jets emerging from this globule that Smith et al. (2010a) identified with $H S T \mathrm{H} \alpha$ imaging. The five separate $\mathrm{HH}$ jet candidates emerging from this elongated globule suggest ongoing star formation from an object dense enough to survive despite harsh UV radiation that has largely cleared the surrounding gas and dust. In general, the $\mathrm{H} \alpha$ features of the candidate $\mathrm{HH}$ jets are too faint or too tenuous to be identified in the ground-based images, although bright $\mathrm{H}_{2}$ and $\mathrm{Br} \gamma$ emission from the head of the globule may reveal the protostar driving $\mathrm{HH}$ c-5.

MHO 1610: MHO 1610 is a single bright, compact $\mathrm{H}_{2}$ knot embedded within more diffuse emission that probably outlines a globule as does so much of the $\mathrm{H}_{2}$ emission from this region of the Eastern Walls (Preibisch et al. 2011b; Area 10 of Figures 5 ; 28). While the images show two other nebulous objects and several stars within the boundaries of the diffuse emission, these sources also appear in the $\operatorname{Br} \gamma$ and $K$-band images and are dominated by continuum. The faint continuum source labeled IRS is coincident with a Herschel point source at $70 \mu \mathrm{m}$. The $\mathrm{H}_{2}$ knot is elongated by about 2.5 arcsec along PA $\sim 45^{\circ}$, and is slightly narrower on its northeastern side.

MHO 1611-1615: MHO 1611, MHO 1612, MHO 1613, MHO 1614, and MHO 1615 form a group of $\mathrm{H}_{2}$ knots located $\sim 8^{\prime}$ northwest of Trumpler 14 (Area 52 of Figures 13; 29). These knots reside near the edge of the dark lane that runs beneath Trumpler 14, and are centered around a loose cluster of at least 20 IR-bright stars. The brightest star at the center of the cluster is PCYC 139, a class II IR source with $L_{\mathrm{BOL}}=400 L_{\odot}$ and an estimated mass of $4.9 M_{\odot}$ (Povich et al. 2011a). MHO 1611 is a group of several faint $\mathrm{H}_{2}$ knots that stretches from near PCYC 139 to near the dark lane to the southwest. MHO 1612, MHO 1613, MHO 1614, and MHO 1615 lie within about a half arcminute of PCYC 139, and could have different 
Table 2

Coordinates for Images

\begin{tabular}{|c|c|c|c|c|}
\hline Object & $\alpha(2000)$ & $\delta(2000)$ & Figure & Notes \\
\hline Area 1 center & $10: 45: 22.07$ & $-59: 58: 48$ & 3 & $\ldots$ \\
\hline Area 2 center & $10: 45: 58.27$ & $-60: 06: 30$ & 3 & $\ldots$ \\
\hline Area 3 center & $10: 43: 50.16$ & $-59: 57: 38$ & 3 & $\cdots$ \\
\hline Area 4 center & $10: 44: 41.04$ & $-59: 57: 37$ & 3 & $\ldots$ \\
\hline Area 5 center & $10: 47: 07.56$ & $-60: 02: 15$ & 3 & $\ldots$ \\
\hline Area 6 center & $10: 45: 13.57$ & $-60: 02: 51$ & 4 & $\cdots$ \\
\hline Area 7 center & $10: 44: 03.21$ & $-60: 07: 00$ & 4 & $\ldots$ \\
\hline Area 8 center & $10: 45: 52.71$ & $-59: 58: 40$ & 4 & $\ldots$ \\
\hline Area 8 inset & $10: 45: 53.73$ & $-59: 57: 02$ & 4 & $\cdots$ \\
\hline Area 9 center & $10: 45: 01.54$ & $-59: 47: 43$ & 5 & $\ldots$ \\
\hline Area 10 center & $10: 46: 03.19$ & $-59: 44: 42$ & 5 & $\ldots$ \\
\hline Area 11 center & $10: 45: 24.30$ & $-59: 45: 37$ & 6 & $\cdots$ \\
\hline Area 12 center & $10: 45: 28.92$ & $-59: 53: 37$ & 6 & $\ldots$ \\
\hline Area 13 center & $10: 46: 05.22$ & $-59: 33: 46$ & 6 & $\cdots$ \\
\hline Area 14 center & $10: 47: 00.47$ & $-59: 37: 49$ & 6 & $\cdots$ \\
\hline Area 15 center & $10: 47: 04.49$ & $-59: 51: 43$ & 7 & $\ldots$ \\
\hline Area 16 center & $10: 45: 58.82$ & $-59: 12: 50$ & 8 & $\ldots$ \\
\hline Area 17 center & $10: 46: 18.41$ & $-59: 14: 27$ & 8 & $\ldots$ \\
\hline Area 18 center & $10: 45: 31.98$ & $-59: 12: 23$ & 8 & $\ldots$ \\
\hline Area 19 center & $10: 44: 07.69$ & $-59: 08: 58$ & 8 & $\cdots$ \\
\hline Area 20 center & $10: 45: 00.73$ & $-59: 21: 60$ & 8 & $\cdots$ \\
\hline Area 21 center & $10: 45: 15.82$ & $-59: 27: 31$ & 9 & $\ldots$ \\
\hline Area 22 center & $10: 44: 01.97$ & $-59: 18: 00$ & 9 & $\ldots$ \\
\hline Area 23 center & $10: 44: 47.22$ & $-59: 27: 21$ & 10 & $\cdots$ \\
\hline Area 24 center & $10: 44: 19.45$ & $-59: 25: 44$ & 10 & $\cdots$ \\
\hline Area 25 center & $10: 44: 22.42$ & $-59: 27: 53$ & 10 & $\cdots$ \\
\hline Area 26 center & 10:44:04.15 & $-59: 33: 42$ & 10 & $\cdots$ \\
\hline Area 27 center & $10: 43: 30.73$ & $-59: 27: 45$ & 10 & $\ldots$ \\
\hline Area 28 center & $10: 44: 33.88$ & $-59: 34: 57$ & 10 & $\ldots$ \\
\hline Area 29 center & $10: 43: 58.22$ & $-59: 29: 44$ & 10 & $\cdots$ \\
\hline Area 30 center & $10: 44: 59.42$ & $-59: 31: 19$ & 10 & $\cdots$ \\
\hline Area 31 center & $10: 43: 38.38$ & $-59: 33: 18$ & 10 & $\ldots$ \\
\hline Area 32 center & $10: 43: 49.54$ & $-59: 37: 40$ & 10 & $\ldots$ \\
\hline Area 33 center & $10: 44: 26.54$ & $-59: 32: 43$ & 10 & $\ldots$ \\
\hline Area 34 center & $10: 45: 07.60$ & $-59: 39: 17$ & 11 & $\ldots$ \\
\hline Area 35 center & $10: 44: 48.08$ & $-59: 37: 39$ & 11 & $\cdots$ \\
\hline Area 36 center & $10: 44: 50.97$ & $-59: 40: 55$ & 11 & $\cdots$ \\
\hline Area 37 center & $10: 44: 31.44$ & $-59: 39: 21$ & 11 & $\ldots$ \\
\hline Area 38 center & $10: 45: 12.50$ & $-59: 37: 41$ & 11 & $\cdots$ \\
\hline Area 39 center & $10: 44: 43.02$ & $-59: 44: 03$ & 11 & $\ldots$ \\
\hline Area 40 center & $10: 44: 03.17$ & $-59: 40: 42$ & 11 & $\ldots$ \\
\hline Area 41 center & $10: 44: 21.10$ & $-59: 42: 04$ & 11 & $\ldots$ \\
\hline Area 42 center & $10: 45: 32.65$ & $-59: 37: 41$ & 11 & $\cdots$ \\
\hline Area 43 center & $10: 43: 41.47$ & $-59: 41: 45$ & 11 & $\cdots$ \\
\hline Area 44 center & $10: 41: 39.21$ & $-59: 43: 33$ & 12 & $\ldots$ \\
\hline Area 45 center & $10: 41: 20.69$ & $-59: 48: 23$ & 12 & $\ldots$ \\
\hline Area 46 center & $10: 42: 43.72$ & $-59: 36: 02$ & 12 & $\cdots$ \\
\hline Area 47 center & $10: 41: 35.31$ & $-59: 35: 07$ & 12 & $\cdots$ \\
\hline Area 48 center & $10: 42: 12.15$ & $-59: 35: 30$ & 12 & $\cdots$ \\
\hline Area 49 center & $10: 41: 54.01$ & $-59: 37: 59$ & 12 & $\ldots$ \\
\hline Area 50 center & $10: 43: 13.16$ & $-59: 37: 24$ & 12 & $\ldots$ \\
\hline Area 51 center & $10: 42: 50.33$ & $-59: 31: 43$ & 13 & $\ldots$ \\
\hline Area 52 center & $10: 42: 36.51$ & $-59: 26: 22$ & 13 & $\cdots$ \\
\hline Area 53 center & $10: 43: 05.53$ & $-59: 32: 36$ & 13 & $\cdots$ \\
\hline Area 54 center & $10: 43: 20.57$ & $-59: 31: 05$ & 13 & $\cdots$ \\
\hline Area 55 center & $10: 42: 58.29$ & $-59: 17: 25$ & 13 & $\cdots$ \\
\hline Area 56 center & $10: 42: 09.74$ & $-59: 32: 52$ & 13 & $\cdots$ \\
\hline Area 57 center & $10: 43: 10.69$ & $-59: 24: 44$ & 13 & $\ldots$ \\
\hline Area 58 center & $10: 41: 12.93$ & $-59: 32: 43$ & 13 & $\cdots$ \\
\hline Area 59 center & 10:43:07.04 & $-59: 29: 04$ & 13 & $\cdots$ \\
\hline Area 60 center & $10: 42: 10.27$ & $-59: 20: 24$ & 13 & $\cdots$ \\
\hline Area 61 center & $10: 43: 26.00$ & $-59: 34: 45$ & 14 & $\cdots$ \\
\hline Area 62 center & $10: 43: 17.13$ & $-60: 02: 51$ & 33 & $\ldots$ \\
\hline Area 63 center & $10: 41: 20.90$ & $-60: 08: 18$ & 33 & $\cdots$ \\
\hline
\end{tabular}

Table 2

(Continued)

\begin{tabular}{|c|c|c|c|c|}
\hline Object & $\alpha(2000)$ & $\delta(2000)$ & Figure & Notes \\
\hline \multicolumn{5}{|c|}{ New Candidate Flows and Associated Objects } \\
\hline HH 1123A & $10: 40: 03.82$ & $-60: 05: 43.1$ & 23 & $\mathrm{H} \alpha,[\mathrm{S}$ II $]$ \\
\hline HH 1123B & 10:40:03.57 & $-60: 05: 44.9$ & 23 & $\mathrm{H} \alpha,[\mathrm{S}$ II $]$ \\
\hline HH $1123 \mathrm{C}$ & 10:40:04.07 & $-60: 05: 46.8$ & 23 & $\mathrm{H} \alpha,[\mathrm{S}$ II $]$ \\
\hline HH 1123D & 10:40:03.66 & $-60: 05: 49.8$ & 23 & $\mathrm{H} \alpha,[\mathrm{S}$ II $]$ \\
\hline $\begin{array}{l}\text { HH } 1124 \\
\quad \text { PCYC } 884\end{array}$ & $10: 45: 28.53$ & $-59: 13: 18.8$ & 24 & Star \\
\hline $\begin{array}{l}\text { HH } 1124 \\
\quad \text { PCYC } 889\end{array}$ & $10: 45: 29.01$ & $-59: 13: 19.5$ & 24 & Star \\
\hline HH 1124 IRS1 & $10: 45: 29.84$ & $-59: 13: 16.1$ & 24 & Star \\
\hline HH 1124 IRS2 & $10: 45: 29.54$ & $-59: 13: 21.0$ & 24 & Star \\
\hline HH 1124A & $10: 45: 31.39$ & $-59: 13: 26.6$ & 24 & $\mathrm{H} \alpha,[\mathrm{S}$ II $]$ \\
\hline HH 1124B & $10: 45: 30.83$ & $-59: 13: 26.4$ & 24 & $\mathrm{H} \alpha,[\mathrm{S}$ II $]$ \\
\hline HH 1124C & $10: 45: 29.07$ & $-59: 13: 22.3$ & 24 & $\mathrm{H} \alpha,[\mathrm{S}$ II $]$ \\
\hline HH 1124D & $10: 45: 26.17$ & $-59: 12: 44.0$ & 24 & $\mathrm{H} \alpha,[\mathrm{S}$ II $]$ \\
\hline HH 1124E & $10: 45: 24.60$ & $-59: 11: 54.9$ & 24 & $\mathrm{H} \alpha,[\mathrm{S}$ II $]$ \\
\hline HH 1125A & $10: 43: 04.18$ & $-59: 55: 01.2$ & 25 & {$[\mathrm{~S}$ II $]$} \\
\hline НH 1125B & $10: 43: 00.44$ & $-59: 54: 52.4$ & 25 & {$[\mathrm{~S}$ II $]$} \\
\hline HHc-21 Star & $10: 44: 10.20$ & $-59: 08: 06.5$ & 32 & $\cdots$ \\
\hline $\begin{array}{l}\text { MHO } 1605 \\
\text { center }\end{array}$ & $10: 43: 51.48$ & $-59: 39: 11.0$ & 10 & $\mathrm{H}_{2},[\mathrm{~S}$ II $]$ \\
\hline IRS 902 & $10: 44: 31.09$ & $-59: 33: 09.6$ & 10 & "Disk Object" \\
\hline MHO 1606 & $10: 44: 22.67$ & $-59: 33: 53.6$ & 10 & $\mathrm{H}_{2},[\mathrm{~S}$ II $]$ \\
\hline MHO 1607 & $10: 44: 28.48$ & $-59: 32: 45.5$ & 10 & $\mathrm{H}_{2}$, east knot \\
\hline MHO 1608 & $10: 44: 29.12$ & $-59: 32: 42.6$ & 10 & $\mathrm{H}_{2}$ \\
\hline MHO 1609a & $10: 45: 09.89$ & $-60: 02: 25.8$ & 27,4 & $\mathrm{H}_{2}, \mathrm{H} \alpha,[\mathrm{S}$ II $]$ \\
\hline MHO 1609b & $10: 45: 10.21$ & $-60: 02: 34.1$ & 27,4 & $\mathrm{H}_{2}, \mathrm{H} \alpha,[\mathrm{S}$ II $]$ \\
\hline MHO 1610 & $10: 46: 08.45$ & $-59: 45: 23.8$ & 28 & $\mathrm{H}_{2}$ \\
\hline MHO 1610 IRS & $10: 46: 07.72$ & $-59: 45: 26.6$ & 28 & $\mathrm{Br} \gamma, \mathrm{H}_{2}$ \\
\hline MHO 1611a & $10: 42: 48.31$ & $-59: 25: 42.1$ & 29 & $\mathrm{H}_{2}$ \\
\hline MHO 1611b & $10: 42: 47.10$ & $-59: 25: 42.8$ & 29 & $\mathrm{H}_{2}$ \\
\hline MHO 1611c & $10: 42: 48.04$ & $-59: 25: 47.3$ & 29 & $\mathrm{H}_{2}$ \\
\hline MHO 1611d & $10: 42: 47.86$ & $-59: 25: 52.1$ & 29 & $\mathrm{H}_{2}$ \\
\hline MHO 1611e & $10: 42: 47.73$ & $-59: 26: 01.8$ & 29 & $\mathrm{H}_{2}$ \\
\hline MHO 1611f & $10: 42: 47.36$ & $-59: 26: 07.8$ & 29 & $\mathrm{H}_{2}$ \\
\hline MHO $1611 \mathrm{~g}$ & $10: 42: 46.94$ & $-59: 26: 13.6$ & 29 & $\mathrm{H}_{2}$ \\
\hline MHO $1611 \mathrm{~h}$ & $10: 42: 45.09$ & $-59: 26: 24.8$ & 29 & $\mathrm{H}_{2}$ \\
\hline MHO $1611 \mathrm{i}$ & $10: 42: 46.11$ & $-59: 26: 29.1$ & 29 & $\mathrm{H}_{2}$ \\
\hline MHO $1611 \mathrm{j}$ & $10: 42: 45.54$ & $-59: 26: 43.6$ & 29 & $\mathrm{H}_{2}$ \\
\hline MHO 1612a & $10: 42: 45.95$ & $-59: 25: 21.8$ & 29 & $\mathrm{H}_{2}$ \\
\hline MHO 1612b & $10: 42: 45.79$ & $-59: 25: 17.7$ & 29 & $\mathrm{H}_{2}$ \\
\hline MHO $1612 \mathrm{c}$ & $10: 42: 45.43$ & $-59: 25: 15.8$ & 29 & $\mathrm{H}_{2}$ \\
\hline MHO 1612d & $10: 42: 45.11$ & $-59: 25: 10.4$ & 29 & $\mathrm{H}_{2}$ \\
\hline MHO 1613 & $10: 42: 46.99$ & $-59: 25: 21.1$ & 29 & $\mathrm{H}_{2}$ \\
\hline MHO 1614a & $10: 42: 49.40$ & $-59: 25: 29.6$ & 29 & $\mathrm{H}_{2}$ \\
\hline MHO 1614b & $10: 42: 48.68$ & $-59: 25: 27.9$ & 29 & $\mathrm{H}_{2}$ \\
\hline MHO 1615a & $10: 42: 47.28$ & $-59: 25: 30.4$ & 29 & $\mathrm{H}_{2}$ \\
\hline MHO $1615 b$ & $10: 42: 46.52$ & $-59: 25: 29.4$ & 29 & $\mathrm{H}_{2}$ \\
\hline MHO 1616a & 10:43:09.13 & $-59: 24: 24.7$ & 13 & $\mathrm{H}_{2}$ \\
\hline MHO 1616b & $10: 43: 09.40$ & $-59: 24: 26.9$ & 13 & $\mathrm{H}_{2}$ \\
\hline MHO $1616 \mathrm{c}$ & $10: 43: 10.30$ & $-59: 24: 37.9$ & 13 & $\mathrm{H}_{2}$ \\
\hline MHO 1616d & $10: 43: 11.30$ & $-59: 24: 46.9$ & 13 & $\mathrm{H}_{2}$ \\
\hline MHO 1616e & $10: 43: 11.50$ & $-59: 24: 48.2$ & 13 & $\mathrm{H}_{2}$ \\
\hline MHO 1616f & $10: 43: 12.70$ & $-59: 24: 59.5$ & 13 & $\mathrm{H}_{2}$ \\
\hline MHO 1616g & $10: 43: 12.95$ & $-59: 25: 01.5$ & 13 & $\mathrm{H}_{2}$ \\
\hline MHO 1617 & $10: 43: 10.22$ & $-59: 24: 53.1$ & 13 & $\mathrm{H}_{2}$ \\
\hline MHO 1617 IR & $10: 43: 09.68$ & $-59: 24: 58.8$ & 13 & $\mathrm{H}_{2}$ \\
\hline MHO 1618 IR & $10: 45: 28.63$ & $-59: 47: 55.1$ & 30 & $\mathrm{H}_{2}$ \\
\hline MHO 1618a & $10: 45: 26.59$ & $-59: 47: 32.6$ & 30 & $\mathrm{H}_{2}$ \\
\hline MHO 1618b & $10: 45: 28.31$ & $-59: 47: 51.2$ & 30 & $\mathrm{H}_{2}$ \\
\hline MHO 1618c & $10: 45: 28.81$ & $-59: 47: 58.5$ & 30 & $\mathrm{H}_{2}$ \\
\hline MHO 1618d & $10: 45: 28.98$ & $-59: 48: 04.4$ & 30 & $\mathrm{H}_{2}$ \\
\hline MHO 1619a & $10: 46: 10.50$ & $-59: 33: 17.9$ & 31 & $\mathrm{H}_{2}$ \\
\hline
\end{tabular}


Table 2

(Continued)

\begin{tabular}{|c|c|c|c|c|}
\hline Object & $\alpha(2000)$ & $\delta(2000)$ & Figure & Notes \\
\hline MHO 1619b & $10: 46: 10.49$ & $-59: 33: 21.6$ & 31 & $\mathrm{H}_{2}$ \\
\hline MHO 1619c & $10: 46: 10.29$ & $-59: 33: 22.4$ & 31 & $\mathrm{H}_{2}$ \\
\hline MHO 1620a & $10: 46: 11.47$ & $-59: 33: 30.6$ & 31 & $\mathrm{H}_{2},[\mathrm{~S}$ II $]$ \\
\hline MHO $1620 \mathrm{~b}$ & $10: 46: 11.99$ & $-59: 33: 30.3$ & 31 & $\mathrm{H}_{2}$ \\
\hline MHO $1620 \mathrm{c}$ & $10: 46: 12.73$ & $-59: 33: 31.4$ & 31 & $\mathrm{H}_{2}$ \\
\hline MHO 1620d & 10:46:13.19 & $-59: 33: 28.4$ & 31 & $\mathrm{H}_{2}$ \\
\hline MHO 1620e & $10: 46: 13.56$ & $-59: 33: 29.6$ & 31 & $\mathrm{H}_{2}$ \\
\hline MHO 1620f & $10: 46: 14.03$ & $-59: 33: 29.2$ & 31 & $\mathrm{H}_{2}$ \\
\hline MHO 1621a & $10: 46: 12.96$ & $-59: 33: 35.5$ & 31 & $\mathrm{H}_{2}$ \\
\hline MHO $1621 \mathrm{~b}$ & $10: 46: 13.18$ & $-59: 33: 36.6$ & 31 & $\mathrm{H}_{2}$ \\
\hline MHO $1621 \mathrm{c}$ & $10: 46: 13.43$ & $-59: 33: 39.3$ & 31 & $\mathrm{H}_{2}$ \\
\hline MHO 1621d & $10: 46: 13.62$ & $-59: 33: 36.9$ & 31 & $\mathrm{H}_{2}$ \\
\hline MHO $1621 \mathrm{e}$ & $10: 46: 13.86$ & $-59: 33: 34.5$ & 31 & $\mathrm{H}_{2}$ \\
\hline PCYC 438 & $10: 44: 07.03$ & $-59: 08: 39.1$ & 32 & Star \\
\hline MHO 1622a & $10: 43: 53.18$ & $-59: 09: 02.5$ & 32 & $\mathrm{H}_{2}$ \\
\hline MHO 1622b & $10: 43: 53.84$ & $-59: 09: 05.4$ & 32 & $\mathrm{H}_{2}$ \\
\hline MHO 1623a & $10: 43: 54.96$ & $-59: 07: 52.8$ & 32 & $\mathrm{H}_{2}$ \\
\hline MHO 1623b & $10: 43: 55.24$ & $-59: 07: 55.8$ & 32 & $\mathrm{H}_{2}$ \\
\hline MHO $1623 \mathrm{c}$ & $10: 43: 56.58$ & $-59: 08: 07.0$ & 32 & $\mathrm{H}_{2}$ \\
\hline MHO 1623d & $10: 43: 57.28$ & $-59: 08: 18.1$ & 32 & $\mathrm{H}_{2}$ \\
\hline MHO 1624a & 10:44:01.93 & $-59: 08: 53.0$ & 32 & $\mathrm{H}_{2}$ \\
\hline MHO 1624b & $10: 44: 03.13$ & $-59: 08: 51.0$ & 32 & $\mathrm{H}_{2}$ \\
\hline MHO $1624 \mathrm{c}$ & $10: 44: 04.31$ & $-59: 08: 51.6$ & 32 & $\mathrm{H}_{2}$ \\
\hline MHO 1625a & 10:44:08.49 & $-59: 09: 03.0$ & 32 & $\mathrm{H}_{2}$ \\
\hline MHO $1625 b$ & 10:44:08.31 & $-59: 09: 08.6$ & 32 & $\mathrm{H}_{2}$ \\
\hline MHO $1625 \mathrm{c}$ & $10: 44: 09.07$ & $-59: 09: 03.9$ & 32 & $\mathrm{H}_{2}$ \\
\hline MHO 1626a & $10: 44: 08.35$ & $-59: 09: 25.9$ & 32 & $\mathrm{H}_{2}$ \\
\hline MHO 1626b & 10:44:09.14 & $-59: 09: 26.4$ & 32 & $\mathrm{H}_{2}$ \\
\hline MHO 1626c & $10: 44: 10.36$ & $-59: 09: 30.0$ & 32 & $\mathrm{H}_{2}$ \\
\hline $\mathrm{R} 1$ & 10:44:21.6 & $-59: 08: 24$ & 32 & $\mathrm{H}_{2},[\mathrm{~S}$ II $], \mathrm{H} \alpha$ \\
\hline $\mathrm{R} 2$ & $10: 44: 11.0$ & $-59: 08: 26$ & 32 & $\mathrm{H}_{2},[\mathrm{~S}$ II $], \mathrm{H} \alpha$ \\
\hline R3 & $10: 44: 11.5$ & $-59: 07: 31$ & 32 & $\mathrm{H}_{2},[\mathrm{~S} \mathrm{II}], \mathrm{H} \alpha$ \\
\hline $\mathrm{R} 4$ & $10: 44: 05.1$ & $-59: 07: 42$ & 32 & $\mathrm{H} \alpha$ \\
\hline R5 & 10:43:58.0 & $-59: 08: 02$ & 32 & $\mathrm{H}_{2},[\mathrm{~S}$ II], $\mathrm{H} \alpha$ \\
\hline MHO 1627a & $10: 41: 14.63$ & $-59: 32: 49.9$ & 13 & $\mathrm{H}_{2}$ \\
\hline MHO 1627b & $10: 41: 14.77$ & $-59: 32: 58.7$ & 13 & $\mathrm{H}_{2}$ \\
\hline MHO 1627c & $10: 41: 15.29$ & $-59: 33: 16.3$ & 13 & $\mathrm{H}_{2}$ \\
\hline MHO 1628a & 10:41:14.15 & $-59: 32: 35.9$ & 13 & $\mathrm{H}_{2}$ \\
\hline MHO 1628b & $10: 41: 14.16$ & $-59: 32: 38.2$ & 13 & $\mathrm{H}_{2}$ \\
\hline PN Candidate & 10:43:34.77 & $-59: 42: 01.5$ & 11 & $\mathrm{H}_{2}$ \\
\hline Finger IRS & $10: 44: 30.16$ & $-59: 38: 40.4$ & 35 & Star \\
\hline SW Loop A & $10: 40: 16.26$ & $-60: 17: 09.7$ & 34 & $\begin{array}{r}\mathrm{H} \alpha, \text { Northern } \\
\text { knot of arc }\end{array}$ \\
\hline SW Loop B & $10: 40: 12.91$ & $-60: 16: 44.7$ & 34 & $\begin{array}{r}\mathrm{H} \alpha, \text { Center of } \\
\text { diffuse area }\end{array}$ \\
\hline SW Loop C & 10:40:05.30 & $-60: 16: 14.4$ & 34 & $\begin{array}{l}\mathrm{H} \alpha \text {, Bright- } \\
\text { est knot }\end{array}$ \\
\hline SW Loop D & 10:40:00.20 & $-60: 16: 12.9$ & 34 & $\begin{array}{l}\mathrm{H} \alpha, \text { Diffuse } \\
\text { structure }\end{array}$ \\
\hline SW Loop E & 10:39:52.62 & $-60: 16: 17.0$ & 34 & $\begin{array}{l}\mathrm{H} \alpha \text {, Middle of a } \\
\text { bright arc }\end{array}$ \\
\hline SW Loop F & 10:39:53.64 & $-60: 14: 09.9$ & 34 & $\mathrm{H} \alpha$ \\
\hline SW Loop G & $10: 39: 47.60$ & $-60: 12: 20.3$ & 34 & $\mathrm{H} \alpha$ \\
\hline
\end{tabular}

exciting sources. MHO 1615 connects with a star situated 2.9 arcsec at $\mathrm{PA}=250^{\circ}$ from PCYC 139. This star appears point-like in the optical images but has an extension along the direction of MHO 1615 in the $\mathrm{Br} \gamma$ and K-band images (Figure 29), hence, it is the likely driving source for that portion of the $\mathrm{H}_{2}$ emission. A faint arc of $\mathrm{H}_{2}$ extends to the east of MHO 1613 toward MHO 1614. Another bright young star

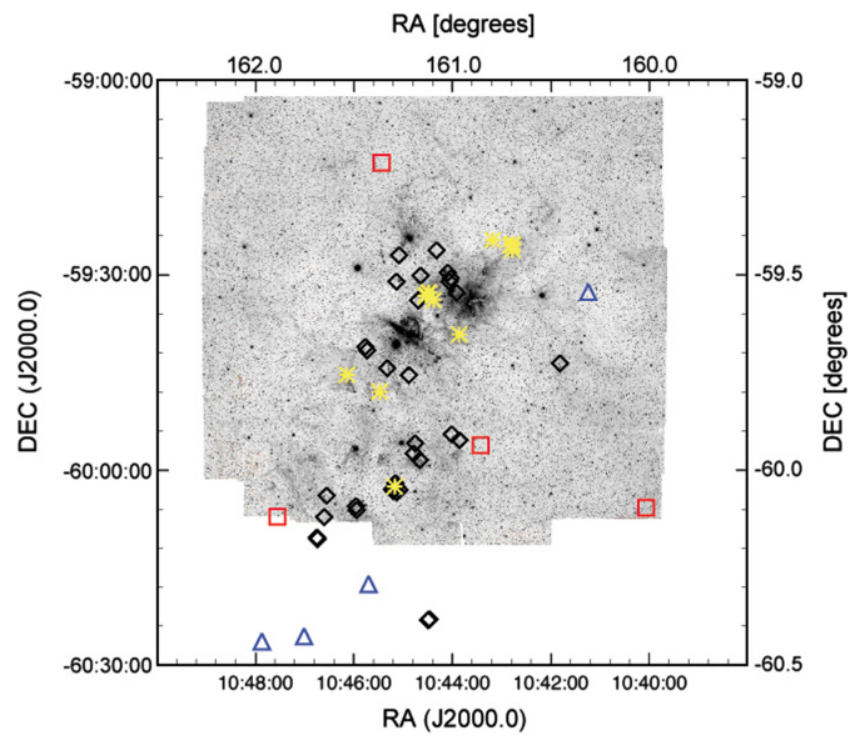

Figure 17. Distribution of candidate jets and previously observed outflows in Carina plotted over the narrowband $\mathrm{H}_{2}$ image. Black diamonds indicate jets previously discovered with HST (Smith et al. 2010a), blue triangles indicate Spitzer EGOs from Smith et al. (2010b), red squares are new candidate jets visible in [S II], and yellow stars are new $\mathrm{H}_{2}$ flow candidates found in this work.

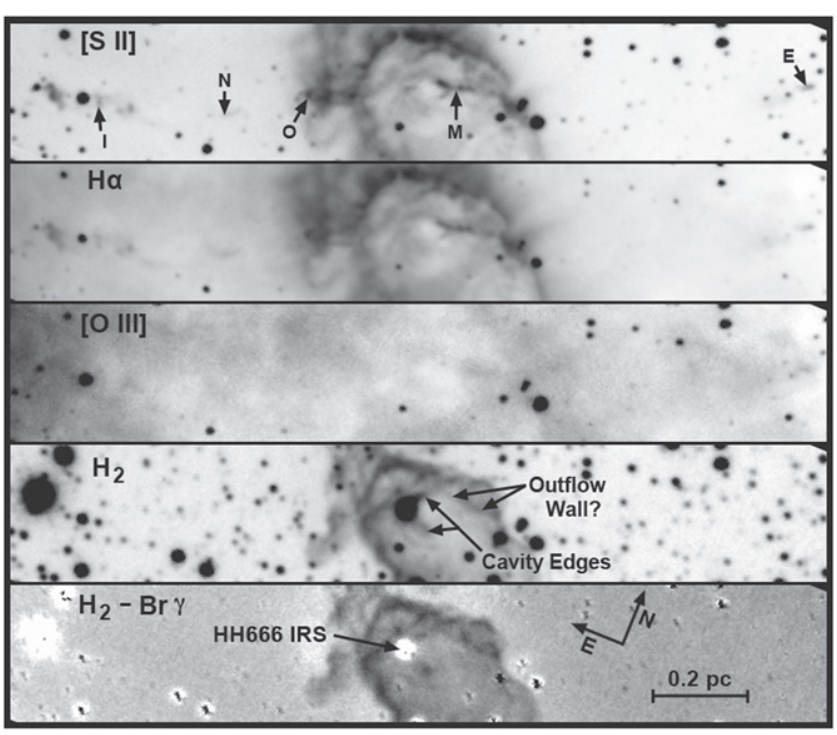

HH 666

Figure 18. Narrowband optical and IR images of HH 666. Labels of the jet features in the $[\mathrm{S}$ II] image follow the nomenclature of Smith et al. (2004a). The $\mathrm{H}_{2}$ emission outlines the edges of a cavity along the jet, and may also trace the northern boundary of the outflow, but no $\mathrm{H}_{2}$ is unambiguously associated with the jet.

and IR source, PCYC 147, is situated 20 arcsec to the east of the IR cluster. Some 6 arcsec to the south of PCYC 147 lies the optically bright B1.5 V star HD 303313, which does not appear heavily extincted and is probably situated in front of the cluster.

The PCYC 139 cluster is not associated with ionized pillars or globules, and is not surrounded by a distinct $\mathrm{H}_{2}$ shell that would outline the photodissociation front into a remnant cloud core. The dark lane to the southwest appears as an $\mathrm{H}_{2}$ wall (Area 52 of Figure 13), but this wall does not have any obvious connection with the cluster. The lack of visual counterparts to the $\mathrm{H}_{2}$ knots suggests that the PCYC 139 cluster lies behind 


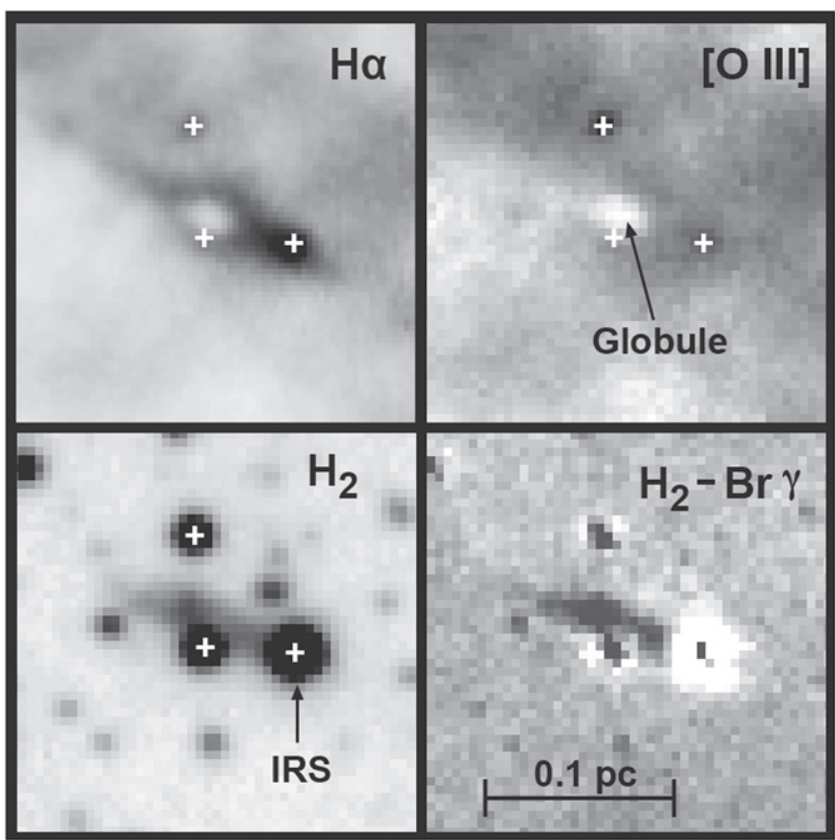

HH 900

Figure 19. Composite of HH 900. The dark globule and IRS (PCYC 838) discussed in the text are marked. Plus-signs denote the location of three bright stars in the region. several magnitudes of extinction on the far side of the Carina Nebula.

MHO 1616-1617: MHO 1616 lies 3 arcmin west of the PCYC 139 cluster, and resembles a clumpy jet (Area 57 of Figure 13). None of the $\mathrm{H}_{2}$ knots have any optical counterparts. While there are several members of the Carina YSO association in the vicinity, none are aligned with the knots of MHO 1616. MHO 1617 is a faint jet of $\mathrm{H}_{2}$ that extends for about 4 arcsec from PCYC 179 at PA $\sim 75^{\circ}$ and ends in a bright knot. Another source 3 arcsec to the south labeled "IR" in Figure 13 has an arcuate morphology that one would expect from a cavity created by an outflow along PA $\sim 50^{\circ}$. This direction intersects with MHO 1617, so it is possible that the IR source is responsible for some of the $\mathrm{H}_{2}$ emission in this region. The IR source falls within the positional errorbars of a Herschel point source at $70 \mu \mathrm{m}$ (Preibisch et al. 2012).

MHO 1618: MHO 1618 is another shocked outflow candidate, this one located in the Eastern Wall region just south of Area 11 (Figure 2). The $\mathrm{H}_{2}-\mathrm{Br} \gamma$ difference image uncovers a faint $\mathrm{H}_{2}$ jet that is aligned with a bright IR source not identified previously as a cluster member (Figure 30). The source is visible in the optical and the IR images and also appears in all four IRAC Bands. We recorded the position of the IR source in Table 2, but it is also possible that the flow originates somewhere to the northwest of knot a, where a dark cloud resides. Proper motions of the $\mathrm{H}_{2}$ knots would settle this question. The putative jet is only visible in $\mathrm{H}_{2}$.

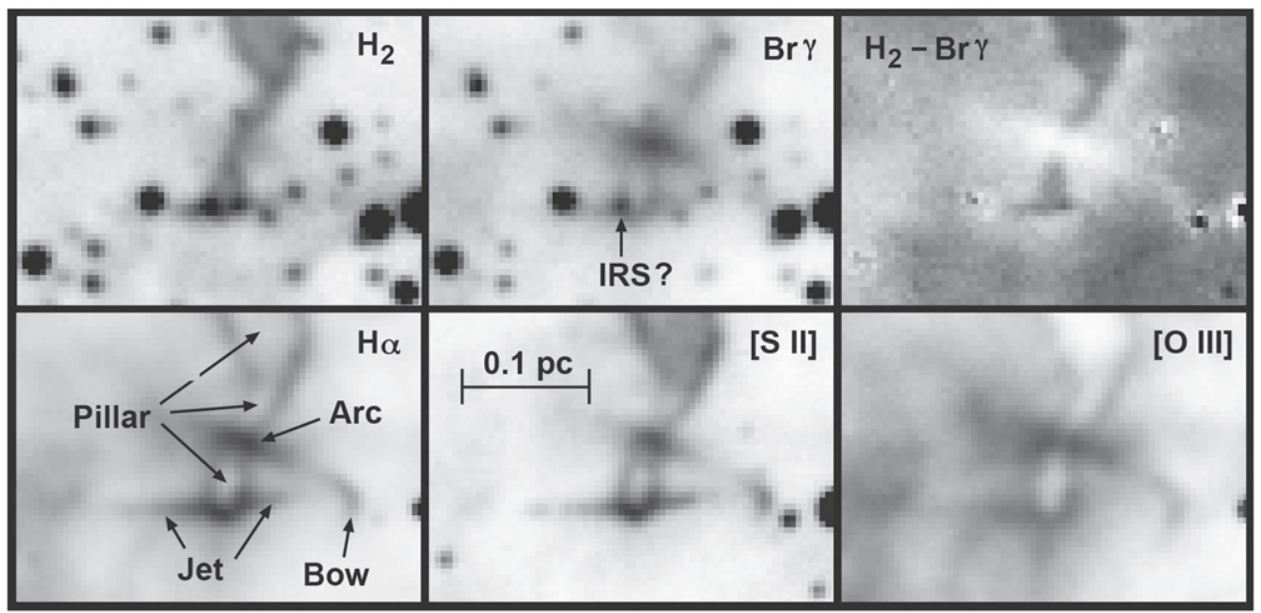

HH 901

Figure 20. Composite of $\mathrm{HH} 901 . \mathrm{H}_{2}$ emission is evident as the jet emerges from the pillar. The objects labeled arc, jet, pillar, and a potential driving source (IRS) are discussed in the text.

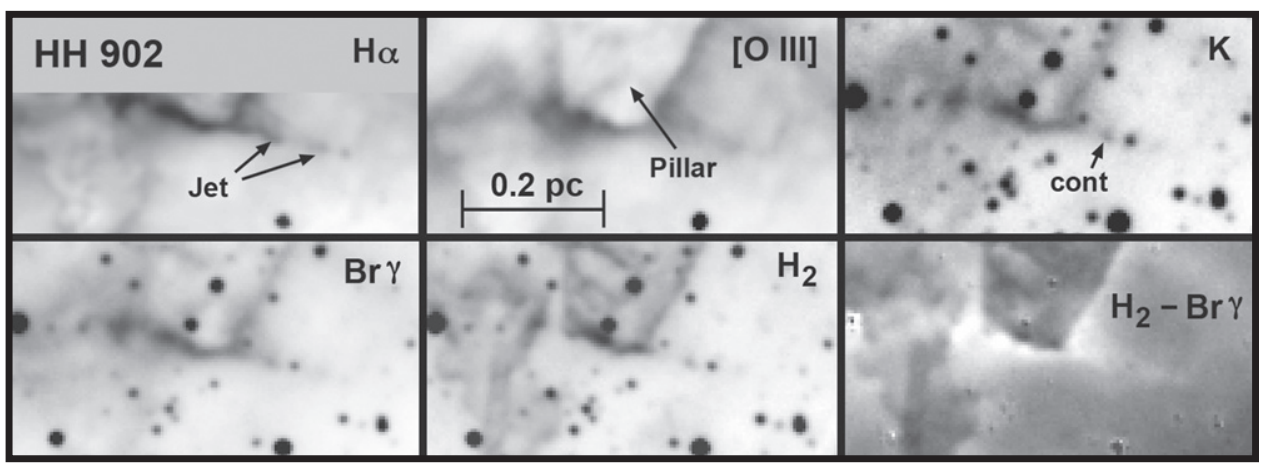

Figure 21. Composite images of the HH 902 region. Labeled objects are described in the text. 


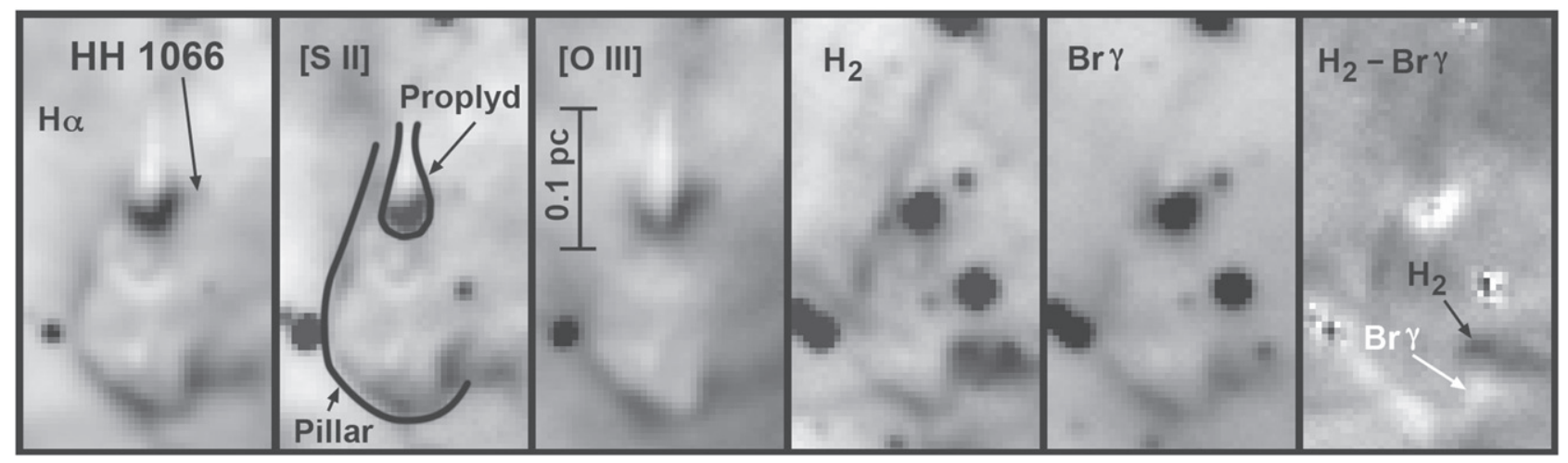

Figure 22. Composite of the proplyd and pillars near HH 1066 (formerly HHc-1). The head of the proplyd shows a distinct C-shape as it is illuminated by stars to the south. Regions marked as bright $\mathrm{Br} \gamma$ and $\mathrm{H}_{2}$ emission are discussed in the text. The star at the head of the proplyd is PCYC 429 .

MHO 1619-1621: a collection of bright $\mathrm{H}_{2}$ clumps labeled MHO 1619, MHO 1620, and MHO 1621 form part of a curve of faint extended $\mathrm{H}_{2}$ situated $\sim 11.5$ arcmin to the northeast of $\eta$ Car (Area 13 of Figures 6 and 31). MHO 1619 is arc-shaped and has faint $\left[\mathrm{S}_{\mathrm{II}}\right]$ emission located to the west-northwest of knot-c. MHO 1620 appears as a chain of knots oriented eastwest. Knot-a is notable for its [S II] emission, and knot-b has a complex internal structure down to the spatial resolution of the images. A third string of $\mathrm{H}_{2}$ knots to the south of MHO 1620 defines MHO 1621. Two stars located on either side of MHO 1621 (Figure 31) have no clear association with the $\mathrm{H}_{2}$. The $\mathrm{H} \alpha$ image of the region shows a filamentary structure in absorption, implying that foreground shells of material exist in this region. None of these MHO objects has a clearly defined exciting source.

G287.24-0.21, PCYC 438, and MHO 1622-1626: Area 19 of Figures 8 and 32 reveal several potential outflow sources and groups of interesting $\mathrm{H}_{2}$ emission line objects. Near the southeast corner of Area 19, a region of extended $\mathrm{H}_{2}$ outlines the boundaries of the molecular cloud G287.24-0.21. The $\mathrm{H}_{2}$ from this region forms a backwards " $y$ " shape in Figure 32 that is also bright in all four IRAC bands in the Spitzer images. The $\mathrm{H} \alpha$ and $\left[\mathrm{S}_{\mathrm{II}}\right]$ emission from G287.24-0.21 are filamentary and more extensive than the $\mathrm{H}_{2}$, but generally follows the $\mathrm{H}_{2}$ shape, as expected for an irradiated cloud boundary.

MHO 1622-MHO 1626 define four groups of clumpy $\mathrm{H}_{2}$ sources in Figure 32. None these show any optical counterparts or obvious driving sources, though the star field in this region is rich and there are several cluster members in this region. MHO 1623, MHO 1624, and MHO 1626 are jet-like in appearance, while MHO 1622 and MHO 1625 have less-collimated morphologies. The bright NIR source PCYC 438 (Povich et al. 2011a; Figure 32) has a bipolar nebula visible in the optical and NIR images that extends $\sim 3$ arcsec on both sides of the star along $\mathrm{PA}=225^{\circ}$, though no jet is visible along the axis of the nebula. The bipolar nebula shows hints of what could be $\mathrm{H}_{2}$ in the $\mathrm{H}_{2}-\mathrm{Br} \gamma$ image, but the nebula is primarily a continuum source.

In addition to the $\mathrm{MHO}$ objects and bipolar nebula described above, five bright arc-shaped $\mathrm{H}_{2}$ and $\mathrm{H} \alpha$ features (labeled R1R5 in Figure 32) define irradiated walls and pillars in this region. These all curve to the north, away from the dominant sources of radiation from the $\operatorname{Tr} 14$ cluster and $\eta$ Car. As in the other Carina PDR interfaces, the $\mathrm{H} \alpha$ emission from the R1-R5 arcs peaks closer to source of ionizing radiation than the $\mathrm{H}_{2}$ emission does.
G287.10-0.73, MHO 1627 and $M H O$ 1628: of the four EGOs found in Spitzer/IRAC images by Smith et al. (2010b), only G287.10-0.73, an elongated IRAC Band 2 source located almost due west of $\operatorname{Tr} 14$, is included within the field of view that we imaged in $\mathrm{H}_{2}$. While we do not see $\mathrm{H}_{2} 2.12 \mu \mathrm{m}$ emission at the location of G287.10-0.73, probably owing to large extinction near the apex of the dusty pillar, our $\mathrm{H}_{2}$ image reveals a striking chain of knots that picks up where the Spitzer extension leaves off and emerges at $\mathrm{PA}=165^{\circ}$ both to the north and to the south from the head of the pillar (Area 58 of Figure 13). On the southern side, a bright $\mathrm{H}_{2}$ jet followed by two knots that are linked together by a stream of faint $\mathrm{H}_{2}$ makes up MHO 1627, while on the northern side, two other bright $\mathrm{H}_{2}$ knots comprise MHO 1628.

Both MHO 1627 and MHO 1628 are visible faintly in the Spitzer images, albeit with poor spatial resolution. No jet is visible in our Mosaic $\mathrm{H} \alpha$, [S II] , or [O III] images. A jet also does not appear in $\mathrm{Br} \gamma$, so that the lack of $\mathrm{H} \alpha$ is not simply due to foreground extinction. A point source at $70 \mu \mathrm{m}$ is located between MHO 1627 and MHO 1628 in Herschel images (Preibisch et al. 2012). The dark pillar associated with G287.10-0.73 is only visible in silhouette. Hence, the ambient ionizing radiation field is not strong in this region, which makes it easier for molecules to survive once the outflow leaves the confines of the dark cloud.

Overall, the number of new candidate flows discovered in Carina with NEWFIRM is smaller than would be expected based on other star-forming complexes at comparable distances (e.g., W5; Ginsburg et al. 2011). Unlike W5, Carina hosts numerous uncovered O-stars that create a harsh radiative environment capable of destroying molecules in much of the central portion of the nebula. The South Pillars region, which extends beyond the boundaries of our NEWFIRM images, has indications of ongoing star formation (e.g., Megeath et al. 1996; Smith et al. 2000; Rathborne et al. 2004; Smith et al. 2010b) that could produce additional flows. The new $\mathrm{H}_{2}$ flows are spatially intermixed with the irradiated jets discovered with HST (Smith et al. 2010a; Figure 17). The outflows are generally most pronounced in either optical $\left(\mathrm{H} \alpha,\left[\mathrm{S}_{\mathrm{II}}\right]\right)$ or molecular $\left(\mathrm{H}_{2}\right)$ emission, but not both simultaneously. None of the molecular jets have strong optical emission and only one of the irradiated jets seen in $\mathrm{H} \alpha$ has appreciable extended $\mathrm{H}_{2}$ emission (see Section 4.1). 


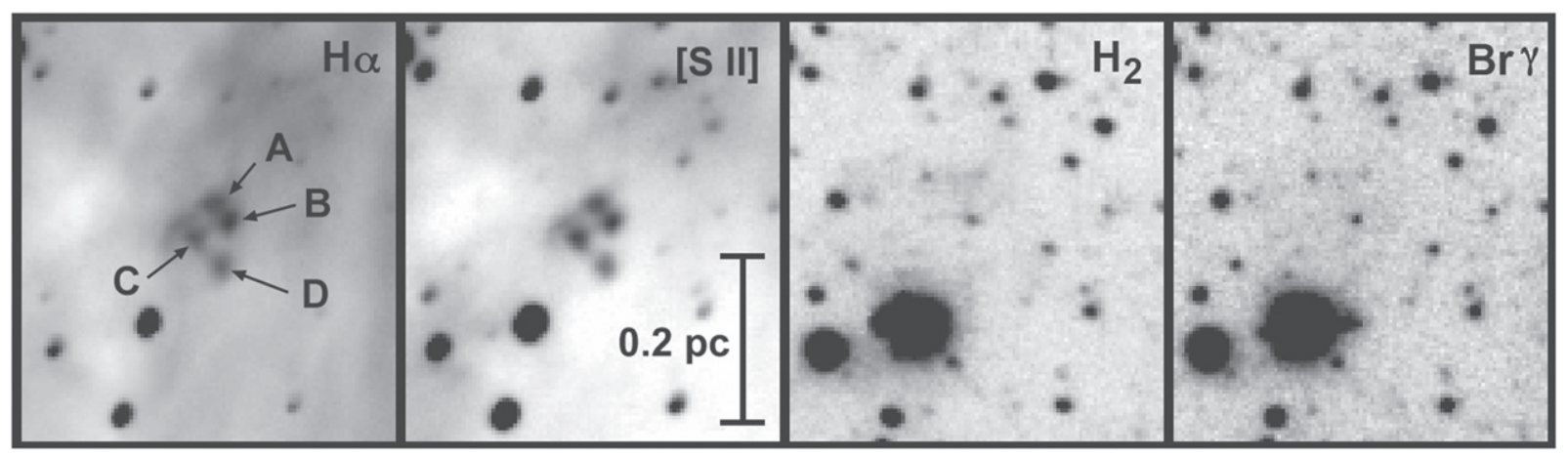

HH 1123

Figure 23. Knots of $\mathrm{H} \alpha$ and $[\mathrm{S}$ II] in $\mathrm{HH}$ 1123. No clear jet structure exists in any of the observed wavelengths.

\subsection{Other Extended Nebulous Objects}

$\mathrm{H}_{2}$ arcs: three $\mathrm{H}_{2}$ arcs to the west of the MHO 1619-1621 group (Area 13 of Figure 6) do not clearly associate with a cloud boundary, and appear bright in $\mathrm{H}_{2}$. These objects look like shell fragments of some sort, but their nature is unclear.

Narrow [S II] filaments in the South Pillars region: The [S II] image and the scaled continuum-subtracted version $[\mathrm{S}$ II $]-\mathrm{I}_{c}$ in Area 62 (Figure 33) reveal a remarkable pattern of five extremely thin, connected filaments. The northeastern filament labeled " 1 " in the figure continues for several parsecs and defines the western boundary of the large pillar that contains the two bright $\mathrm{H}_{2}$ structures in Area 3 (Figures 2, 3). The curved shape of the cloud is visible faintly to the west of filament 3. The southern portion of filament 3 also emits weakly in $\mathrm{H} \alpha$. Filaments 2-4 are most easily explained as constituting the leading edge of a bubble that is impinging upon the cloud (see inset to Figure 33). In this model filament 5 could define the southern edge of the cloud. Proper motion measurements would be able to verify or disprove this scenario, which predicts motion of filaments $2-4$ to be northward, eastward, and southward, respectively.

The intersection points between shock fronts have generated a fair bit of interest recently because under some circumstances a normal shock known as a Mach stem may develop at this location, leading to a hot spot that has anomalous pattern motion relative to the larger bow shock structure. Multipleepoch HST images show evidence for time-variable enhanced emission at intersection points of bow shocks exists in $\mathrm{HH} 34 \mathrm{~S}$ and possibly also $\mathrm{HH}$ 47A (Hartigan et al. 2011). Laboratory experiments of this phenomena have quantified the critical angles where Mach stems can form (Foster et al. 2010; Yirak et al. 2013), and the experiments agree reasonably well with the theoretical and numerical predictions.

In Figure 33, there is a small bright segment where filaments 3-5 intersect, but no Mach stem should form there because the angle between filaments 4 and 5 is too acute, and none exists in the object. The situation at the junction of filaments 1-3 is more complex. One bright $\left[\mathrm{S}_{\mathrm{II}}\right]$ knot, two fainter ones, and several foreground stars lie near the intersection point. The projected angle between filaments 1 and 2 is $75^{\circ}-105^{\circ}$, depending on how one interprets the spatial structure at that point. An angle in the $75^{\circ}-90^{\circ}$ range may produce a Mach stem, but the shock is nearly normal to the interface in any case. Filament 3 is distinctly more wavy in appearance than are the adjacent filaments, as expected if this filament marks the location of the shock as it overtakes irregularities along the surface of the cloud. The brightness variations along filament 3 seem uncorrelated with whether or not the wiggles in the front are concave or convex.

Large [S II] shells in the South Pillars region: a region where several bright arcs of $[\mathrm{S}$ II] overlap may define a fossil cavity driven by the massive stars to the north (Area 63 of Figure 33). The shells extend east-west for over a parsec and are visible faintly in the $\mathrm{H} \alpha$ images though not in [O III].

Shells and filaments in the Southwest Loop: the southwestern boundary of the bright optical emission in the Carina Nebula is defined by a large curved arc that spans several parsec in size, and within the arc lie a number of smaller knots and filaments. This region lies to the southwest of our $\mathrm{H}_{2}$ mosaic, but was covered by the optical Mosaic images (see Figure 34). The $\mathrm{I}_{c^{-}}$ band image shows very little continuum along the arc. The scaled $\mathrm{H} \alpha-\mathrm{I}_{c}$ images in Figure 34 remove stars reasonably well, and show that the arc has several bright knots situated along its inner part (labeled A-G in Figure 34), as well as what appear to be smaller cavities (features H, I, and J) embedded in its outer portion. Knots $\mathrm{C}$ and $\mathrm{F}$ are bright and clumpy, and emit strongly in $[\mathrm{S} \mathrm{II}]$.

The arc has the morphology and line emission characteristics of an expanding bubble that is ionized by massive stars at the center of the Carina Nebula. Features A-G appear to mark locations where the arc is impacted by winds that originate from the active central region of the Nebula. The existence of distinct smaller shells within the arc argues for multiple wind sources. Meaburn \& Walsh (1986) discovered very similar structures within the Rosette, so this phenomenon is not unique to the Carina Nebula.

Candidate background planetary nebula: Area 43 in Figure 11 shows limb-brightened, elliptical $\mathrm{H}_{2}$ feature with some wisps extending beyond the ellipse along a perpendicular axis. The object is unusual for Carina in that $\mathrm{Br} \gamma$ emission fills the $\mathrm{H}_{2}$ ellipse, and weak $\mathrm{H} \alpha$ is also present. A faint star is located near the center of the object. An IRAC Band 2-Band 1 difference image reveals some extended $4.5 \mu \mathrm{m}$ (green) emission in the center of this object. IRAC emission matches the $\mathrm{Br} \gamma$ emission, but not $\mathrm{H}_{2}$, suggesting we are seeing $\mathrm{Br} \alpha$ and not shocked $\mathrm{H}_{2}$ in the $4.5 \mu \mathrm{m}$ image in this case. The emission structure with $\mathrm{H}_{2}$ surrounding $\mathrm{Br} \gamma$ emission, combined with a symmetrical elliptical morphology and a central star is best explained by a planetary nebula situated behind the obscuring dark clouds of the Carina Nebula. 

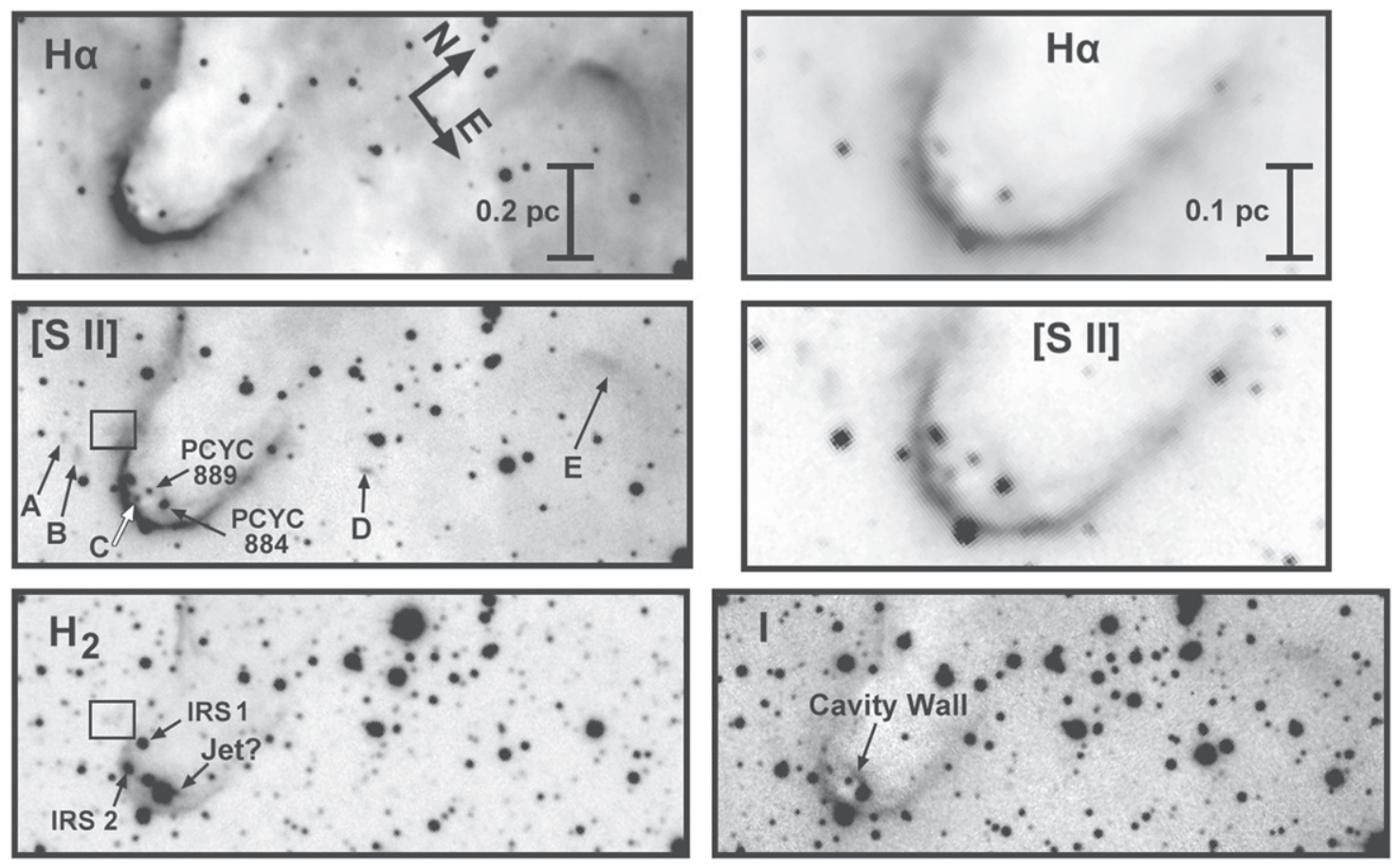

HH 1124

Figure 24. Optical emission-line knots A-C outline a bent flow that emerges to the west of the head of the pillar shown in Area 18 of Figure 8 . Knot D and the bowshaped object $\mathrm{E}$ lie to the east. The source labeled IRS (PCYC 884) shows what appears to be a cavity wall in reflected continuum. An extension in the $\mathrm{H}_{2}$ image (also visible in the $\mathrm{H}_{2}-\mathrm{Br} \gamma$ image in Figure 8) may outline a jet, but it could also define a photodissociation surface on the irradiated pillar. Emission in the boxed region is discussed in the text.

Knots near the Carina "Defiant Finger": a striking irradiated globule known as the Carina Defiant Finger (Smith et al. 2004b) exists in the vicinity of the Keyhole. A chain of emission knots extends from the globule's northern edge (from the "wrist") that are bright in $\mathrm{H} \alpha,[\mathrm{S} \mathrm{II}]$, and $\mathrm{H}_{2}$ (Area 37 of Figures $11 ; 35)$. However, the knots exhibit spatial offsets between $\left[\mathrm{S}\right.$ II] and $\mathrm{H} \alpha$, and between $\mathrm{H}_{2}$ and $\mathrm{Br} \gamma$ characteristic of PDRs. The [O III] image shows the knots in absorption against the nebular background, as expected for dusty globules. Faint emission from the knots in the IRAC bands is consistent with thermal emission from these dusty blobs. This collection of objects most likely define the remains of a pillar within Trumpler 16 that has been destroyed by the intense UV radiation in this region. The northern-most knot has a bright IR source on its northern boundary labeled as "IRS" in Figure 35. This location is where a young star should be if it formed at the apex of the pillar. Smith et al. (2004b) derived a photoablation rate of $\dot{M} \approx 2 \times 10^{-5} M_{\odot} \mathrm{yr}^{-1}$ in this region, so small knots such as these should be transient objects.

\section{The Runaway Star Trumpler 14 MJ 218}

The $\mathrm{Br} \gamma$ images reveal a curious arc-shaped feature situated about 6 arcsec to the northwest of a bright star (Area 26). The arc stands out in color composites owing to its relatively strong $\mathrm{Br} \gamma$ emission as compared with $\mathrm{H}_{2}$ (Figure 10). The star, located at 10:44:05.1 -59:33:41 (2000.0), was first observed photometrically by Feinstein et al. (1973) and later spectroscopically by Massey \& Johnson (1993), and is listed in the Reed (2003) catalog of O and B stars. The above references named the star Trumpler 1429 and Trumpler 14 MJ 218, respectively. The stellar colors are consistent with a reddened early B star, and the high-quality blue spectrum (Massey \& Johnson 1993) shows a spectral type of B1.5 V.

The arc has recently been discussed in some detail by Ngoumou et al. (2013), who refer to it as the "Sickle" (see also Figure 1 of Ascenso et al. 2007). As shown by Ngoumou et al. (2013), the star appears to be moving past a dense clump observed in $870 \mu \mathrm{m}$ images, and the shape and offset of the bow shock from the stellar image is consistent with a wind from the star impacting denser material in the vicinity of the globule. The star has a large proper motion of $8.7 \mathrm{mas} \mathrm{yr}^{-1}$ at $\mathrm{PA}=307^{\circ}\left(95 \mathrm{~km} \mathrm{~s}^{-1}\right.$ at a distance of $\left.2.3 \mathrm{kpc}\right)$, consistent with producing the bow shock (Zacharias et al. 2013), and is most likely a runaway star ejected as its companion underwent a supernova explosion. However, errors in proper motion measurements at the distance of Carina are relatively large, and if the X-ray radiation from this source arises from a companion, it may be difficult to keep the binary system intact if a more massive primary became a supernova. Our difference image $\left(\mathrm{H}_{2}-\mathrm{Br} \gamma\right)$ in Figure 10 shows enhanced $\mathrm{H}_{2}$ along a flattened structure that lies along the portion of the bow closest to the star, as one would expect if molecular hydrogen in the shell were excited either by the shock or by UV fluorescence from the star.

Extrapolating forward, Tr 14 MJ 218 should move a degree on the sky in about $4 \times 10^{5} \mathrm{yr}$, which amounts to $\sim 10 \%$ of the star-forming epoch within a typical molecular cloud. Carina hosts $\sim 70 \mathrm{O}$ stars, which typically become supernovae after 5-10 million years (Meynet \& Maeder 2003). Many of these O stars are binaries, with the companions being future runaway stars. Hence, we expect at most a few runaway stars to exist within the Carina Nebula at any given time. We note that one neutron star has been discovered in the Carina region, implying 


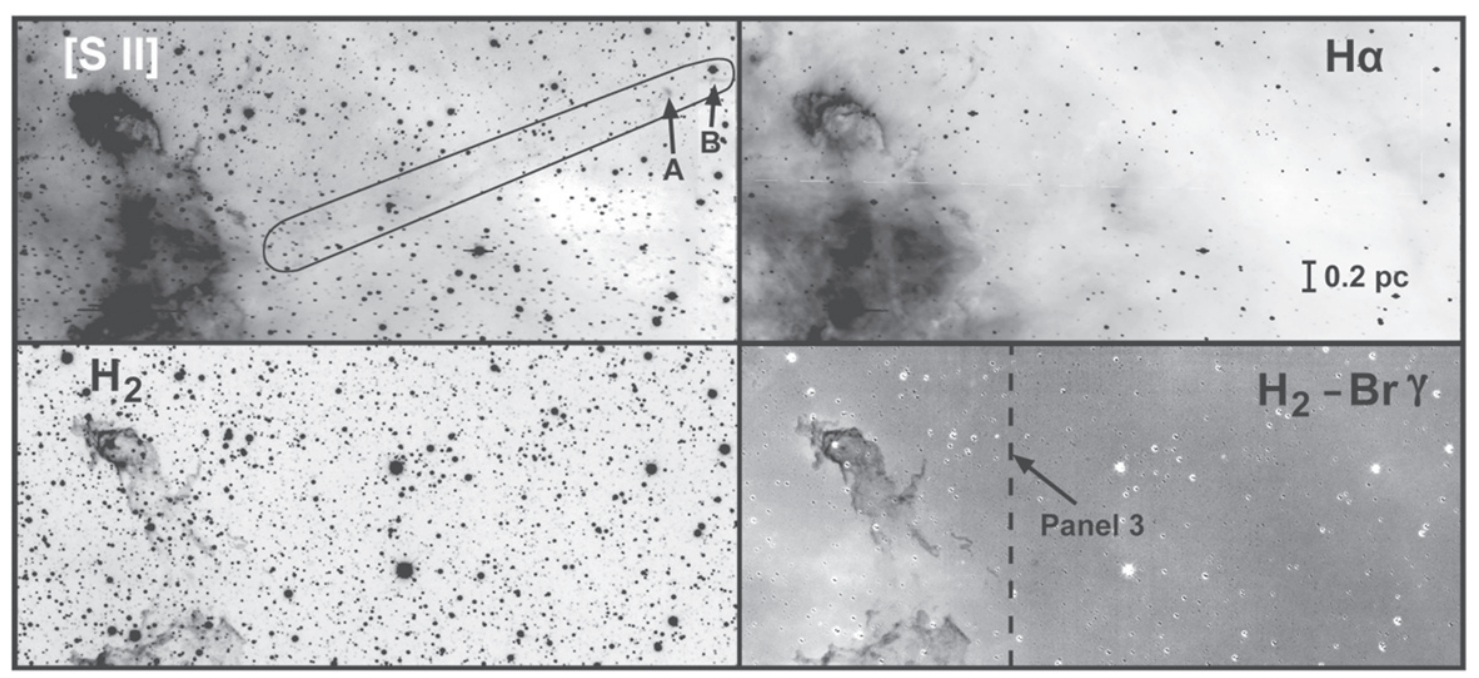

HH 1125

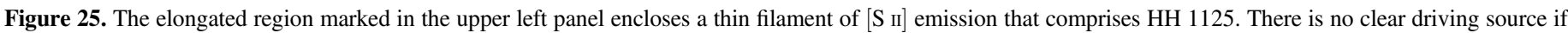

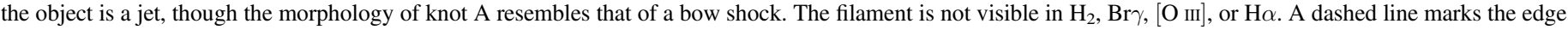
of Area 3 in Figure 3.

recent supernova activity (Hamaguchi et al. 2009), and abundance variations of heavy metals across the nebula also point to recent supernovae (Hamaguchi et al. 2007).

Tr 14 MJ 218 represents the only clear candidate for a runaway star in our field-many emission-line arcs appear in our images, but the bow shock ahead of this star is unusual in that is has a smooth, diffuse shape, a large $\mathrm{Br} \gamma-\mathrm{to}-\mathrm{H}_{2}$ ratio, and a bright star contained within the bow. This object, together with eight other early-type stars in Carina, were highlighted by Smith et al. (2010b) as having extended emission in $8 \mu \mathrm{m}$ Spitzer images and classified as extended red objects (EROs). Two of these EROs lie to the south of our mosaics, while the other six appear as unremarkable point sources in the $\operatorname{Br} \gamma$ and $\mathrm{H}_{2}$ images.

\section{5. $\mathrm{H}_{2}$ WITHIN EMBEDDED CLUSTERS}

The Carina star-formation region is sometimes referred to as a "cluster of clusters"; as many as 24 separate clusters were identified in the recent deep CCCP X-ray survey Feigelson et al. (2011). The Trumpler 16 region (near $\eta$ Car) alone consists of least six smaller clusters, and as many as eight were identified from Spitzer data (Smith et al. 2010b). While $\mathrm{H}_{2}$ walls and pillars often exist in the vicinity of these clusters, most clusters visible in the optical and NIR are no longer embedded within their nascent molecular cloud cores.

However, two young clusters stand out in our images in that they have what appear to be cavities defined by $\mathrm{Br} \gamma$ emission at the cluster's center that is surrounded by circular arcs of $\mathrm{H}_{2}$ that mark the boundary of the cavities evacuated by the cluster stars. The most notable of these from the standpoint of $\mathrm{H}_{2}$ emission is undoubtedly the Treasure Chest (Area 8 in Figure 4). This region was described in some detail by Smith et al. (2005). The star at the center of the $\operatorname{Br} \gamma$ emission is an O9.5 V star named CPD $-59^{\circ} 2661$, and this object excites the emission lines within the local $\mathrm{H}$ II region that surrounds it. A bright emission-line star (Sanduleak \& Stephenson 1973; Hen 3-485 in Area 8) lies to the upper left of the cluster, and was identified as a likely Herbig Be star on the basis of its X-ray flux by Gagne et al. (2011). The NIR observations of Preibisch et al. (2011a) show that about one-third of the stars in the Treasure Chest have NIR excesses indicative of circumstellar disks, a fraction about three times larger than occurs in the rest of the Nebula.

The narrowband images in Figure 3 of Smith et al. (2005) show that faint $\mathrm{Pa} \beta$ extends northward from the $\mathrm{H}_{2}$ emission in the Treasure Chest, in the direction of $\eta$ Car and $\operatorname{Tr} 16$. We also observe this behavior in the reddening-independent $\mathrm{H}_{2}-\mathrm{Br} \gamma$ difference image in Area 8 of Figure 3. Bright $\mathrm{Br} \gamma$ emission defines the $\mathrm{H}$ II region excited by the cluster, and a sinuous arc of $\mathrm{H}_{2}$ outlines the PDR from the cluster. Hence, the dark cloud in which the Treasure Chest resides is undergoing photodissociation both internally from the embedded cluster, and externally from $\eta$ Car and other young stars within $\operatorname{Tr} 16$.

Another cluster of interest that has $\mathrm{H}_{2}$ emission is shown in Area 30 (Figure 10). Discovered independently by Preibisch et al. (2011b), it was not identified by Feigelson et al. (2011) as either a major cluster or as a smaller group. While less spectacular than the Treasure Chest, this cluster contains 30-40 stars and is surrounded by filamentary $\mathrm{H}_{2}$ emission. The brightest NIR source is G 287.51-0.49, located at 10:44:59.70 $-59: 31: 19.5$, is also a mid-IR source (Rathborne et al. 2004; Sanchawala et al. 2007), though the lack of a prominent H II region in $\mathrm{Br} \gamma$ implies it is not a powerful source of ionizing radiation.

\section{DISCUSSION: FEEDBACK, TRIGGERING, AND PDR PHYSICS IN THE CARINA COMPLEX}

\subsection{Feedback}

Carina is among the most active regions of star formation within several kpc of the Sun. The region contains more than 70 stars of spectral type $\mathrm{O}$, has a total mass of young stars of $M_{\text {stars }} \simeq 4 \times 10^{4} M_{\odot}$ and a neutral gas mass of at least $M_{\mathrm{gas}} \simeq$ $(0.5-1) \times 10^{6} M_{\odot}$ (Smith 2006b; Smith \& Brooks 2007; Preibisch et al. 2011b; Preibisch et al. 2011c). The kinetic energy of the expanding nebula is about $8 \times 10^{51} \mathrm{ergs}$, about 


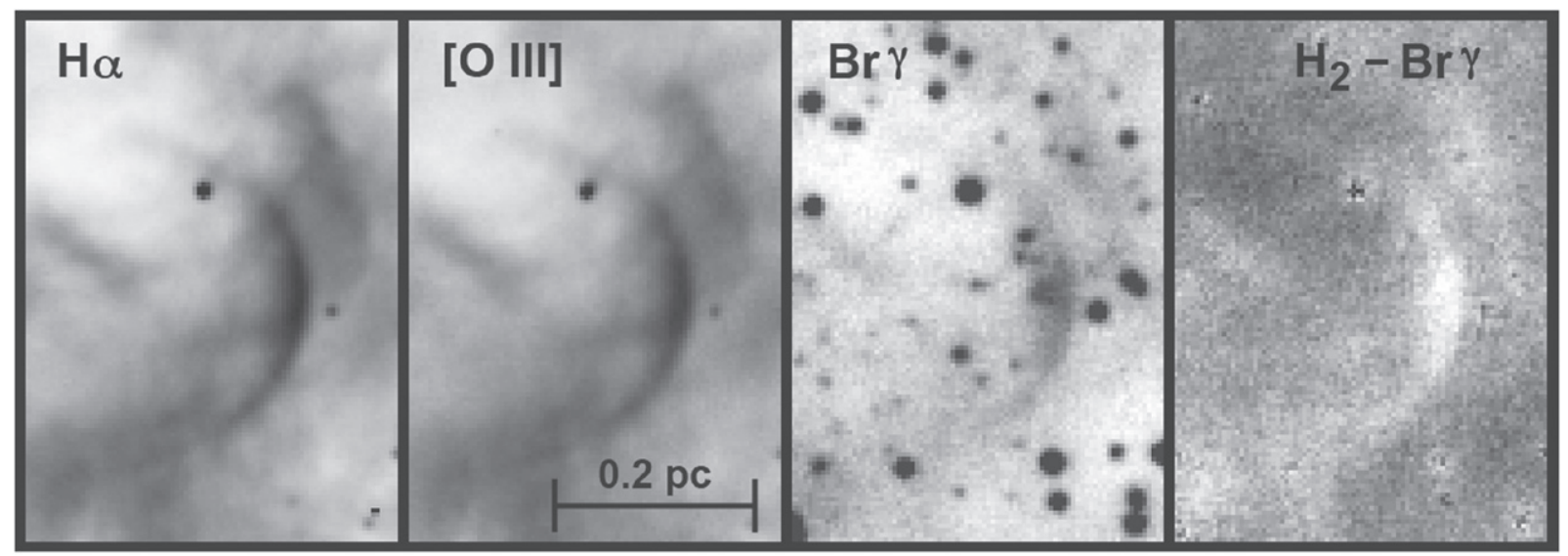

HH c-9

Figure 26. Highly ionized arc-shaped $\mathrm{HH}$ c-9 is bright in $\mathrm{H} \alpha$ and $\left[\mathrm{O}\right.$ III], and has a similar morphology in $\mathrm{Br} \gamma$. No $\mathrm{H}_{2}$ is present in this object. If the object is a bow shock, its driving source remains unidentified.

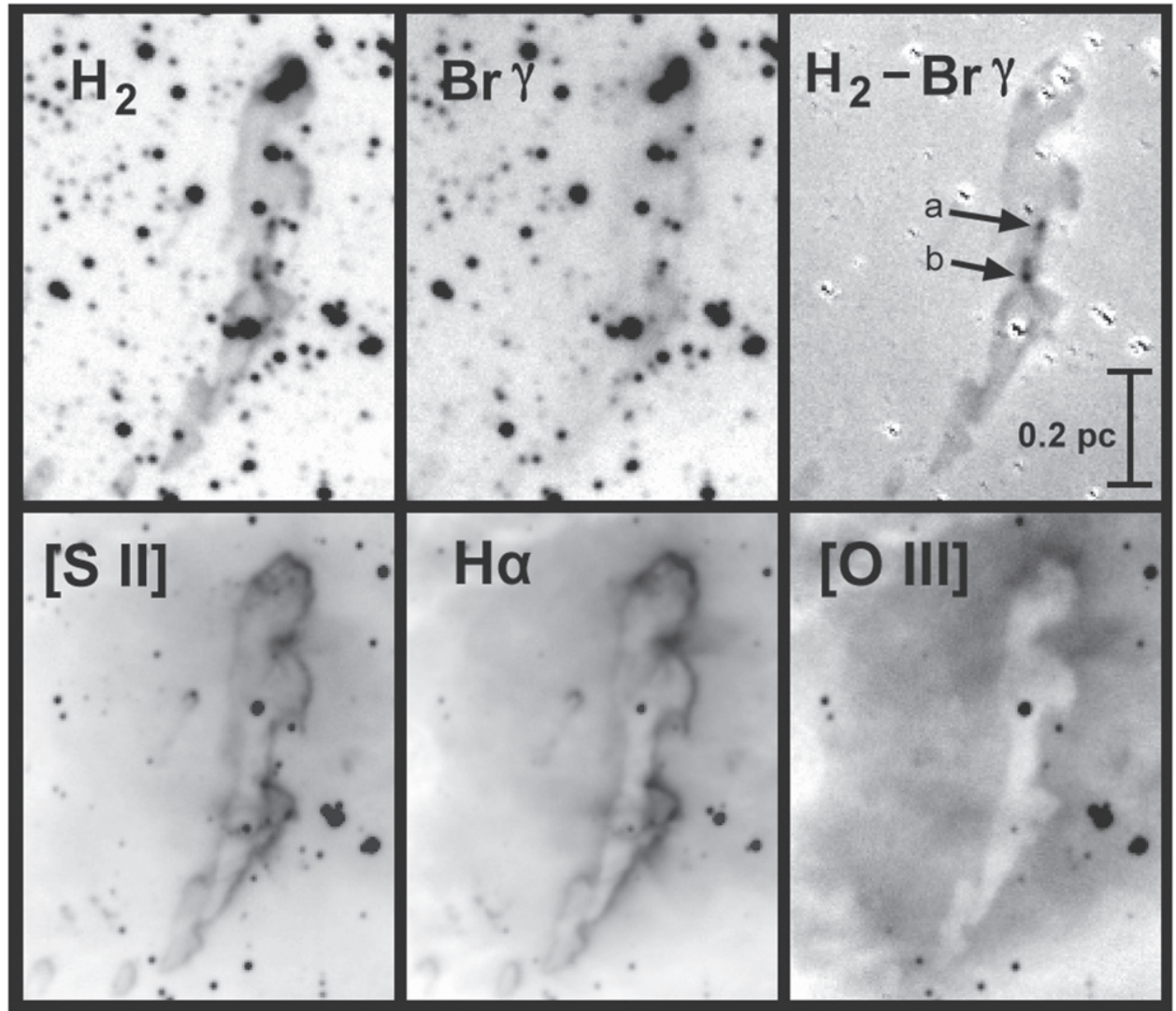

MHO 1609

Figure 27. Two bright, extended $\mathrm{H}_{2}$ features within an irradiated pillar comprise MHO 1609. Several optical outflow candidates were identified from this detached globule in the HST data.

$1 / 3$ of the mechanical energy available from stellar winds in the past 3 Myr (Smith \& Brooks 2007).

Feedback from the many young massive stars in the Carina Nebula has had a profound influence on the surrounding interstellar medium. These massive stars have cleared a cavity and sculpted dozens of gas pillars that face the center of the Nebula. The detailed structure of these pillars and other cloud formations stand out in the $\mathrm{H}_{2}$ images presented in our paper because the $\mathrm{H}_{2}$ images trace the thin PDRs on the surfaces that outline these irradiated clouds. The relatively high subarcsecond angular resolution provided by these images complements the longer wavelength data that trace the warm dust and molecular gas inside the clouds seen in previous surveys with IRAS, MSX, Spitzer, and CO data (Yonekura et al. 2005; Smith \& Brooks 2007; Smith et al. 2010b).

Recently Roccatagliata et al. (2013) analyzed the impact of feedback in Carina using ground-based APEX and space-based data (Preibisch et al. 2011b) and Herschel Space Observatory 


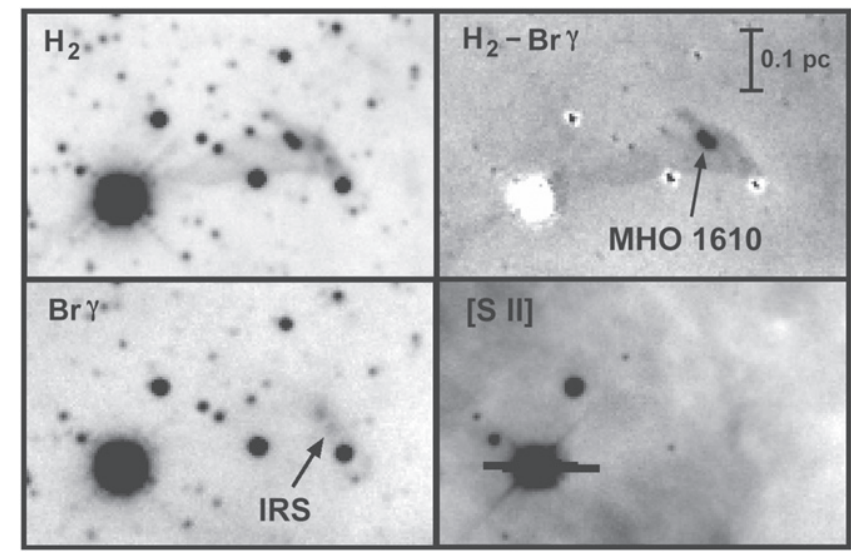

Figure 28. Composite images of MHO 1610 (see also Area 10 of Figure 5) reveal a single bright $\mathrm{H}_{2}$ knot with no optical counterpart. A Herschel point source at $70 \mu \mathrm{m}$ is coincident with the faint continuum source labeled IRS.

\subsection{Triggering (or Not)?}

There are three main conceptual scenarios that describe how young massive stars affect star formation in their neighborhoods: (1) "pre-existing uncovered" star formation (PRE-SF), where winds and radiation from massive stars evacuate material in the region and reveal stars that have already formed in multiple locations throughout the molecular cloud, (2) "external pressure-induced collapse" of pre-existing clouds that would not otherwise have formed stars in isolation (e.g., induced by radiation, winds, or supernovae; EPIC-SF), and (3) "collect and collapse" star formation (CC-SF), where the external agent is also responsible for forming the parent cloud (see, e.g., Elmegreen \& Lada 1977; Bertoldi 1989).

Although our new images of Carina provide a rich harvest of phenomena associated with stars currently forming under the influence of external feedback, the degree to which this star formation activity was actually triggered by that feedback

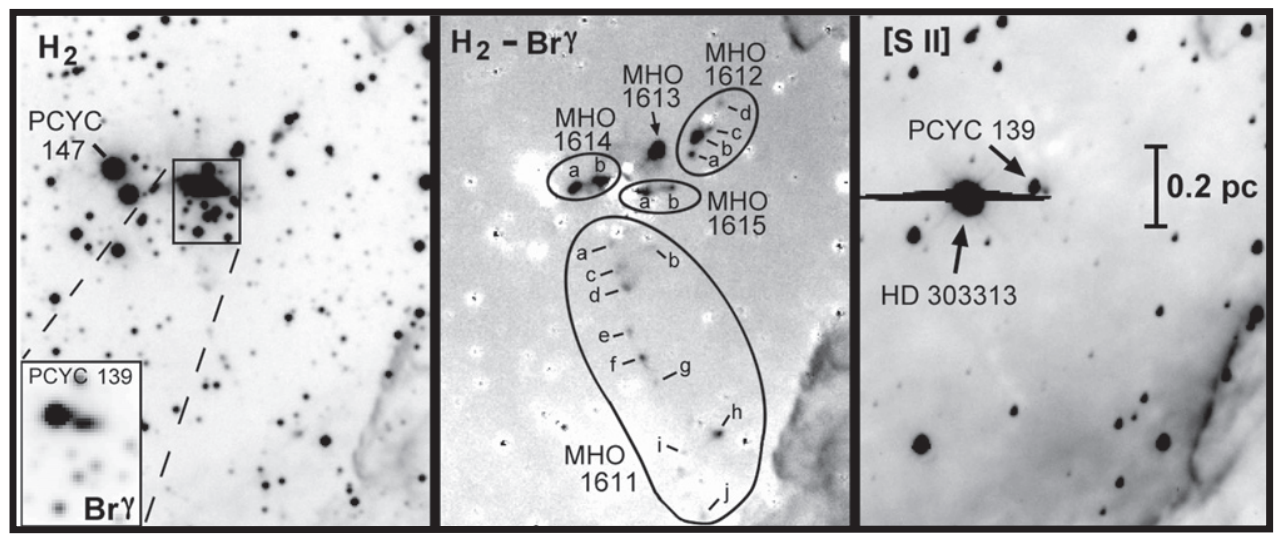

MHO 1611 - 1615

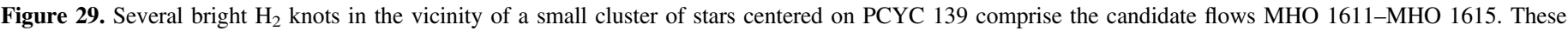

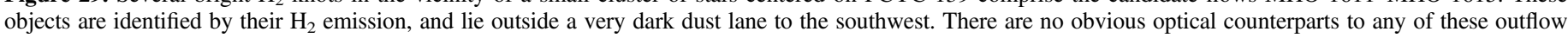
candidates.

sub-mm observations of Preibisch et al. (2012). However, disentangling the emission from the thin PDRs and protostars embedded within them that emit at long wavelengths remains challenging because emission from the PDR and the protostar are not resolved from one-another at long wavelengths. Perhaps this is a task for ALMA, and to this end our new $\mathrm{H}_{2}$ images will provide a reliable reference for the structure of the PDRs over the entire region.

Some authors have proposed that the initial formation of the massive stars in Carina was itself triggered by external influences. Maps of the molecular gas surrounding the Carina Nebula reveal a CO supershell containing about $8 \times 10^{2} M_{\odot}$ of $\mathrm{H}_{2}$, the Carina Flare, which appears to be blowing out of the Galactic Plane (Fukui et al. 1999; Dawson et al. 2008a, 2008b; Wünsch et al. 2012). Emission in the $21 \mathrm{~cm} \mathrm{H}$ I line from the Carina Flare and 10-100 $\mu \mathrm{m}$ dust emission also uncover a shell that extends up to $10^{\circ}$ above the Galactic plane with a mass of at least $2 \times 10^{5} M_{\odot}$. Fukui et al. (1999) propose that energetic feedback inflated a $300 \times 400 \mathrm{pc}$ suberbubble that expands with a mean velocity of about $10 \mathrm{~km} \mathrm{~s}^{-1}$. These authors propose that the Carina Flare was produced by massive stars that formed about $120 \mathrm{pc}$ above the Galactic mid-plane, and that the impact of their energy release may have triggered star formation in the current Carina Nebula. remains quite unclear. This situation echoes many discussions of morphological evidence for triggered star formation in other regions; despite abundant wide-field imaging data, the questions persist and have been debated for decades. Our images show that the Carina Nebula contains dozens of pillars with cometary tails that trail away from Carina's massive stars, and in nearly all cases there is a young star with an IR excess embedded at the head of the pillar, sometimes driving an outflow. Although morphologies of this kind have been interpreted as evidence for triggered star formation, this geometry could also arise as winds and radiation from massive stars uncover star formation events that would have occurred anyway in the absence of any triggering effects.

To demonstrate that triggering occurs in any given region is a difficult problem, requiring numerical simulations with multidimensional radiation hydrodynamics, and perhaps magnetic fields. Currently, models have not provided decisive observational tests to determine if star formation is triggered based on the morphology of the gas and protostars, and in the absence of such discerning tests, morphology alone as seen in our images cannot be used to support or refute triggering. If future theoretical studies can clarify specific morphologies that are or are not due to triggering, the $\mathrm{H}_{2}$ images presented here may be quite valuable to address this question. 


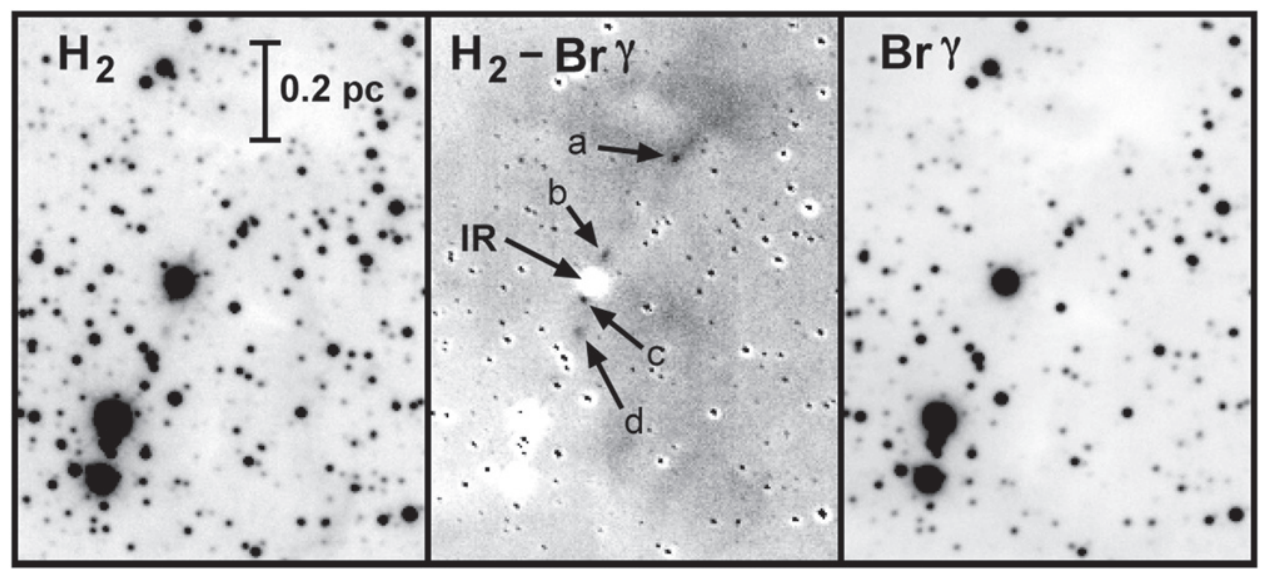

MHO 1618

Figure 30. Situated in the Eastern Walls, the candidate outflow MHO 1618 appears as a curving string of $\mathrm{H}_{2}$ knots on either side of an infrared source.

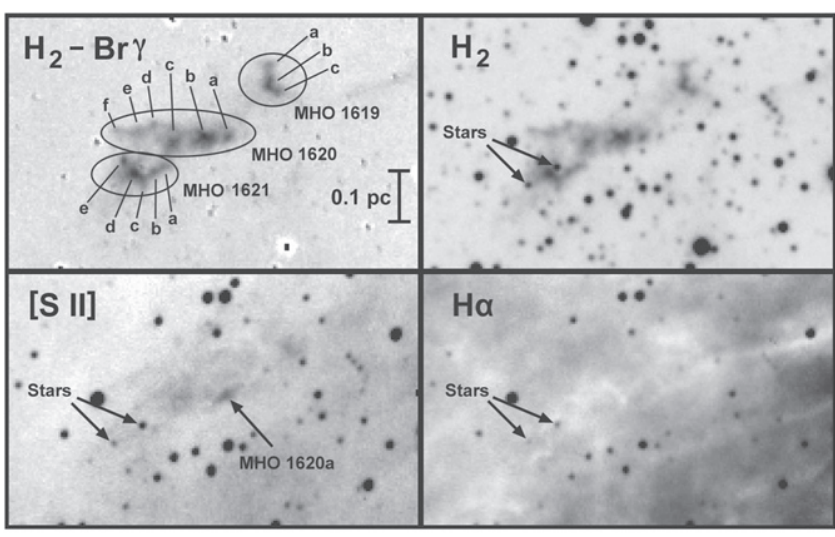

MHO 1619 - 1621

Figure 31. Composite images of MHO 1619-MHO 1621 (see also Area 13 of Figure 6). MHO $1620 \mathrm{a}$ is visible in the $[\mathrm{S}$ II] image. No driving source is present.

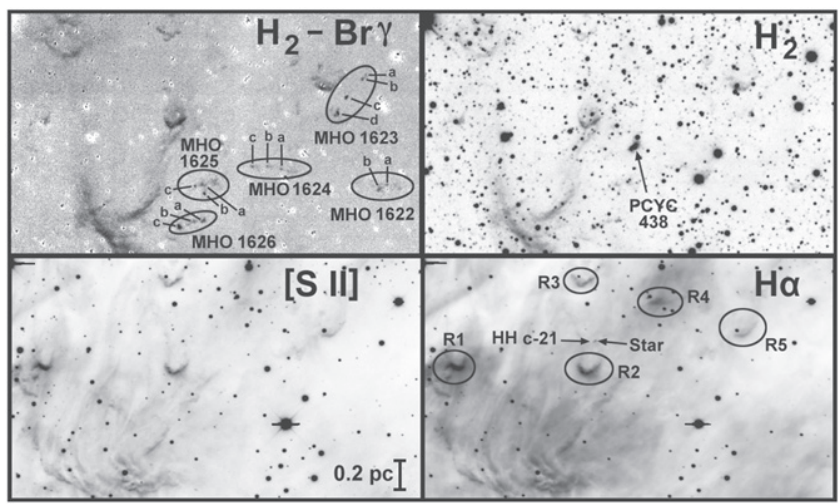

MHO 1622 - 1626

Figure 32. Composite images in the G287.24-0.21 cloud, showing several strings of $\mathrm{H}_{2}$ knots groups as MHO 1622-MHO 1626 (see also Area 19 of Figure 8). The arc-shaped sources R1-R5 mark locations of irradiated pillars.

In the meantime, detailed investigations of age gradients in the stars as compared to gas, as well as star and gas kinematics may be more fruitful avenues to investigate triggering, although we cannot address these with the present data set. Here too, though, clear predictions of ages or age-gradients for triggered star formation as opposed to uncovered (and posibly truncated)
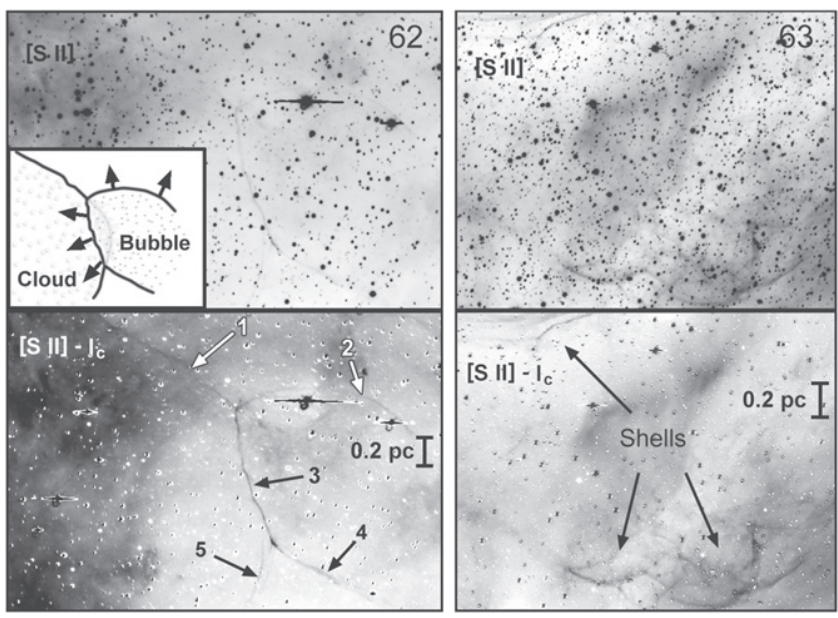

Figure 33. Left: a network of five narrow filaments is visible in the $\left[\mathrm{S}_{\mathrm{II}}\right]$ image (top), and the continuum-subtracted image (bottom). The model described by the inset is discussed in the text. Right: Area 63 shows an extensive network of overlapping shells in the $[\mathrm{S}$ II $]$ images. They are oriented as if driven by the massive stars to the north of this region.

star formation are not obvious. The upcoming GAIA mission will have 10-100 micro arcsec proper motion sensitivity, which for Carina and other nearby regions, has the potential to distinguish pre-existing uncovered star formation from triggered star formation, at least among the young stars already exposed at visual wavelengths. Protostars still embedded in Carina's pillars, young clusters such as the Treasure Chest (Smith et al. 2005), and the driving sources of active outflows such as HH 666 exhibit large IR excesses, indicating ages less than about $1 \mathrm{Myr}$. In contrast, the massive stars that have evolved to the LBV or red supergiant phases, and the "naked" portions of clusters such as Tr 15 must be at least $3 \mathrm{Myr}$ old. But, as noted above, it remains possible that the younger stars and clusters embedded in the surviving molecular clouds of Carina would have formed anyway in the absence of the older generation.

In the case of the Treasure Chest, our $\mathrm{H}_{2}$ images (Section 5; Area 8 of Figure 4) show an external PDR produced by radiation from the massive stars in the environment, as seen previously, but they also reveal an internal PDR that marks the boundary of where the embedded cluster stars dissociate 


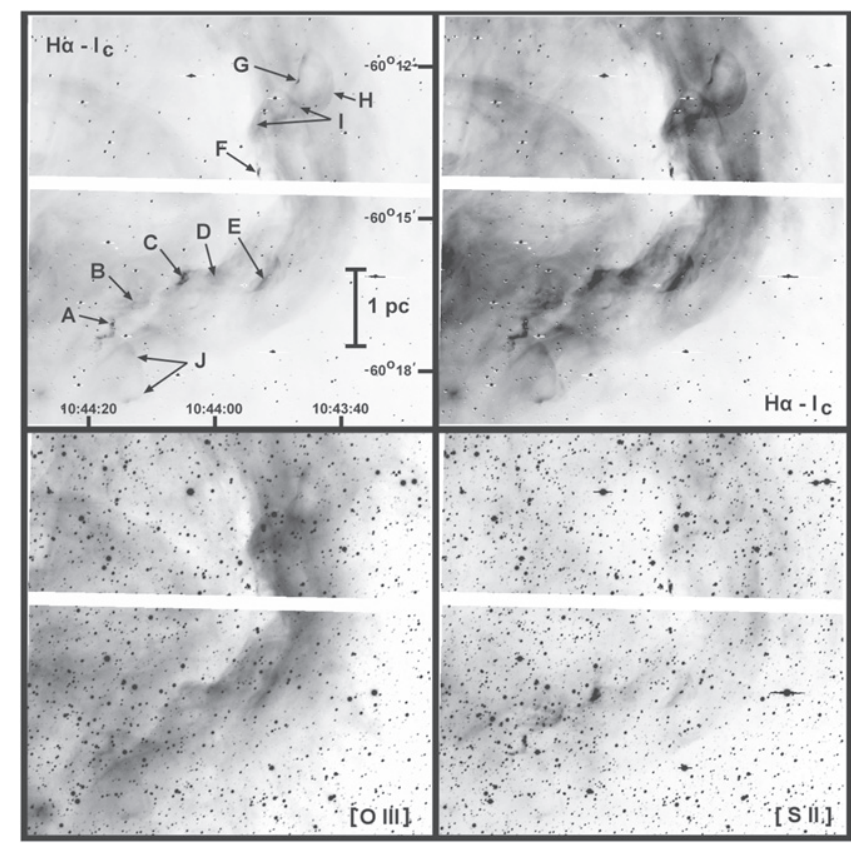

Southwest Loop

Figure 34. Cavities, filaments and knots in the Southwestern Loop, located at the edge of the bright optical emission in Carina. Top: $\mathrm{H} \alpha$ images with scaled $\mathrm{I}_{c}$ continnum subtracted, presented at two different grayscales. Bottom: a narrowband $[\mathrm{O}$ III] image (left) and $[\mathrm{S} \mathrm{II}]$ image (right).

molecules in their natal cloud core. Forming such a structure by triggering is only viable if the lifetime of the core after it is exposed by the triggering event-in this case radiation and winds from the nearby massive stars-is long enough to allow for an entire cluster to form and to create its own internal $\mathrm{H}$ II region and PDR. This complex problem should be addressed numerically in light of this observational constraint.

\subsection{The Carina Nebula as a Laboratory for PDR Physics}

Models of PDRs typically specify the FUV radiation flux $G_{\text {。 }}$ $(6-13.6 \mathrm{eV})$ and the particle density $n$, and then track how the molecular, atomic, and ionic elemental abundances vary with depth into the molecular cloud. Most theoretical formalisms use the visual extinction $A_{V}$ as a convenient independent variable, with the boundary of the $\mathrm{H}$ II region at $A_{V} \lesssim 1$, followed by the PDR at larger $A_{V}$. Specific zones exist within a PDR: models show a transition from $\mathrm{H}$ to $\mathrm{H}_{2}$ at $A_{V} \sim 2, \mathrm{C}^{+}$to $\mathrm{C}$ and $\mathrm{CO}$ at $A_{V}$ $\sim 4$, and more complex chemistry ongoing at larger depths (Tielens \& Hollenbach 1985; Hollenbach et al. 1991).

Models of $\mathrm{H}_{\text {II }}$ regions require less chemistry, and are correspondingly more sophisticated than their PDR counterparts when it comes to dynamics such as R- and D-fronts (Henney et al. 2005), 3D capability, magnetic fields, and timedependence (Arthur et al. 2011), turbulence (Medina et al. 2014), and photoevaporation from pillars (Ercolano et al. 2011). These H II region models also produce synthetic emission-line images that have a direct connection to observations. Merged models of $\mathrm{H}_{\text {II }}$ regions and PDRs developed by assuming pressure equilibrium between the PDR and $\mathrm{H}_{\text {II }}$ region as a first approximation to the dynamics are a relatively recent theoretical development (Abel et al. 2005; Kaufman et al. 2006), and fractal models of PDRs are under development (Andree et al. 2013).

Our observations of $\mathrm{H}_{2} 1-0 \mathrm{~S}(1),[\mathrm{S} \mathrm{II}], \mathrm{Br} \gamma$ and $\mathrm{H} \alpha$ probe the boundary between the $\mathrm{H}$ II region and the PDR and show clear offsets in the spatial locations of these lines (Figure 16). While modern PDR models include $\mathrm{H}_{2}$ (1-0) S1 emission from fluorescent processes, and $\mathrm{H}$ II region calculations include $\mathrm{Br} \gamma$, $\mathrm{H} \alpha$, [ $\mathrm{S} \mathrm{II}]$, and $[\mathrm{O} \mathrm{III}]$ line cooling, we are not aware of any simulations that predict synthetic emission maps for all these lines in a single simulation, even for a $1 \mathrm{D}$ model. It should be possible to produce such maps for both a planar geometry and pillars with existing codes. For example, motivated by new

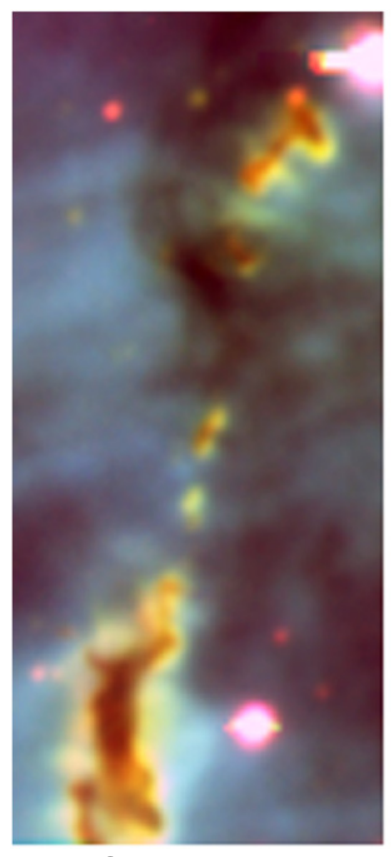

Composite

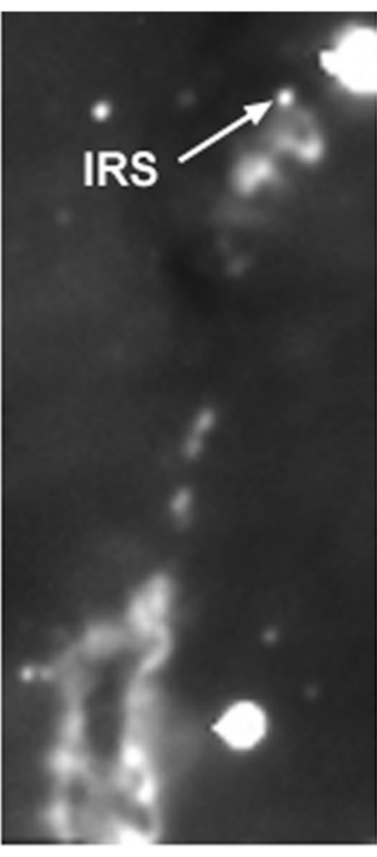

r: [S II]

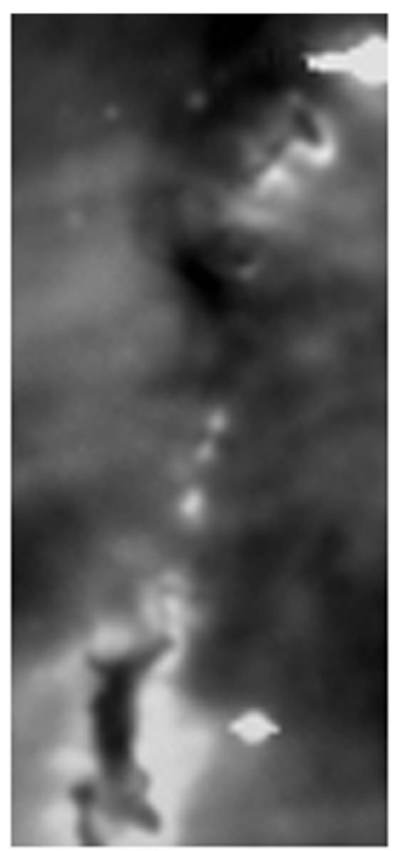

$\mathrm{g}: \mathrm{Ha}$

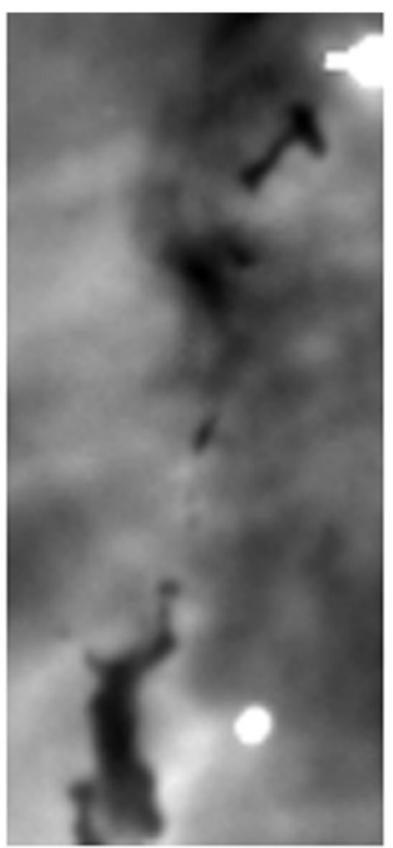

b: [O III]

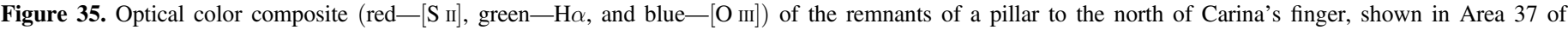
Figure 11. The bright near-IR source labeled IRS is discussed in the text. The emission lines arise from PDRs at the surface of the knots. 
Spitzer mid-IR spectra, Kaufman et al. (2006) made predictions for rotational $\mathrm{H}_{2}$ 0-0 lines along with forbidden lines of $\mathrm{Si}$ II, Fe II, and C II. Relative to the Spitzer data, our observations give an order-of-magnitude improvement in spatial resolution and reliable photocenter offsets between the $\mathrm{H}_{2}, \mathrm{H}$, and $\mathrm{S}$ II lines. Simply predicting photocenter offsets of all the bright emission lines in an $\mathrm{H}_{\mathrm{II}}$ region/PDR interface in a $1 \mathrm{D}$ model as a function of parameters such as densities, radiation fields, ages, and even magnetic field strengths would be the easiest and most direct comparison with the data in Figure 16.

\section{SUMMARY}

We have presented a survey of the $\mathrm{H}_{2}$ and $\mathrm{Br} \gamma$ emission line sources over a square degree in size centered on the Carina Nebula using the NEWFIRM camera at the CTIO $4 \mathrm{~m}$ telescope with total integration times on-source of 12.5 and $8.7 \mathrm{ksec}$, respectively, and subarcsecond seeing. Our NIR images are complemented by new narrowband optical images at $\left[\mathrm{O}\right.$ III], $\mathrm{H} \alpha$, and $\left[\mathrm{S}_{\mathrm{II}}\right]$, as well as a suite of broadband images. The new data clarify the morphologies of irradiated interfaces such as pillars, walls, and globules, identify jets, outflows and flared disks, and provide insights into the star formation activity present within embedded clusters. We also quantified spatial offsets that are present between various emission lines along the irradiated interfaces, and compared each region with existing far-IR and X-ray images.

In many ways, the Carina Nebula is an ideal region to study how massive stars affect their surroundings. The large number of O-stars, combined with relatively low extinction and moderate distance makes it possible to study star formation on a variety of scales. Surveys such as ours provide an overview of the entire region, and also identify specific objects that are worthy of followup with higher-resolution imaging and spectroscopy with HST and ground-based AO systems. The high radiation environment in a region like Carina in some ways makes it easier to interpret outflows, pillars, and walls because all irradiated dense regions become visible in emission-line images. This advantage is particularly evident in fluorescent lines such as $\mathrm{H}_{2}$. However, in some regions projection of multiple irradiated surfaces makes interpretation difficult. Future work centered on time-domain observations such as high-precision proper motions should help to clarify the dynamics of the stars and gas in this remarkable region.

We thank Jacob Palmer for his assistance with the NEWFIRM data acquisition at the $4 \mathrm{~m}$, and the CTIO and NOAO staff for their efforts in getting the instrument operational. Megan Reiter would like to thank Andy Marble for his help generating the jet distribution plot. We thank Bo Reipurth and Chris Davis for quickly providing new $\mathrm{HH}$ and $\mathrm{MHO}$ object numbers, and an anonymous referee for helpful suggestions regarding the presentation and organization of the paper. The PI was funded by DOE through the NLUF program during the course of this project.

\section{REFERENCES}

Abel, N. P., Ferland, G. J., Shaw, G., \& van Hoof, P. A. M. 2005, ApJS, 161,65

Andree, S., Röllig, M., \& Ossenkopf, V. 2013, in ASP Conf. Ser. 476, New Trends in Radio Astronomy in the ALMA Era, ed. R. Kawabe, N. Kuno, \& S. Yamamoto (San Francisco, CA: ASP), 311
Arthur, S. J., Henney, W. J., Mellema, G., de Colle, F., \& Vazquez-Semadeni, E 2011, MNRAS, 414, 1747

Ascenso, J., Alves, J., Vicente, S., \& Lago, M. T. V. T. 2007, A\&A, 476, 199

Bally, J., Johnstone, D., Joncas, G., Reipurth, B., \& Mallén-Ornelas, G. 2001, AJ, 122, 1508

Bertoldi, F. 1989, ApJ, 346, 735

Brooks, K. J., Burton, M. G., Rathborne, J. M., Ashley, M. C. B., \& Storey, J. W. V. 2000, MNRAS, 319, 95

Brooks, K. J., Cox, P., Schneider, N., et al. 2003, A\&A, 412, 751

Brooks, K. J., Storey, J. W. V., \& Whiteoak, J. B. 2001, MNRAS, 327, 46

Broos, P. S., Townsley, L. K., Feigelson, E. D., et al. 2011, ApJS, 194, 2

Davis, C., Gell, R., Khanzadyan, T., Smith, M. D., \& Jenness, T. 2010, A\&A, 511, A24

Dawson, J. R., Kawamura, A., Mizuno, N., Onishi, T., \& Fukui, Y. 2008a, PASJ, 60, 1297

Dawson, J. R., Mizuno, N., Onishi, T., McClure-Griffiths, N. M., \& Fukui, Y. 2008b, MNRAS, 387, 31

Donehew, B., \& Brittain, S. 2011, AJ, 141, 46

Elmegreen, B. G., \& Lada, C. J. 1977, ApJ, 214, 725

Ercolano, B., Dale, J. E., Gritschneder, M., \& Westmoquette, M. 2011, MNRAS, 420, 141

Gagne, M., Fehon, G., Savoy, M. R., et al. 2011, ApJS, 194, 5

Ginsburg, A., Bally, J., \& Williams, J. P. 2011, MNRAS, 418, 2121

Feigelson, E. D., Getman, K. V., Townsley, L. K., et al. 2011, ApJS, 194, 9

Feinstein, A., Marraco, H. G., \& Muzzio, J. C. 1973, A\&AS, 12, 331

Foster, J. M., Rosen, P. A., Wilde, B. H., Hartigan, P., \& Perry, T. S. 2010, $\mathrm{PhPl}, 17112704$

Fukui, Y., Onishi, T., Abe, R., et al. 1999, PASJ, 51, 751

Ghosh, S. K., Iyengar, K. V. K., Rengarajan, T. N., et al. 1988, ApJ, 330,928

Grenman, T., \& Gahm, G. F. 2014, A\&A, 565, A107

Hamaguchi, K., Corcoran, M. F., Ezoe, Y., et al. 2009, ApJL, 695, L4

Hamaguchi, K., Robert, P., Matsumoto, H., et al. 2007, PASJ, 59, 151

Hartigan, P., Frank, A., Foster, J. M., et al. 2011, ApJ, 736, 29

Hartigan, P., Palmer, J., \& Cleeves, L. I. 2012, HEDP, 8, 313

Harvey, P. M., Hoffmann, W. F., \& Campbell, M. F. 1979, ApJ, 227, 114

Henney, W. J., Arthur, S. J., Williams, R. J. R., \& Ferland, G. J. 2005, ApJ, 621,328

Hester, J. J., Scowen, P. A., Sankrit, R., et al. 1996, AJ, 111, 2349

Hollenbach, D. J., Takahashi, T., \& Tielens, A. G. G. M. 1991, ApJ, 377, 192

Hollenbach, D. J., \& Tielens, A. G. G. M. 1997, ARA\&A, 35, 179

Hur, H., Hwankyung, S., \& Bessell, M. S. 2012, AJ, 143, 41

Kaufman, M. J., Wolfire, M. G., \& Hollenbach, D. J. 2006, ApJ, 644, 283

Lee, H.-T., Liao, W.-T., Froebrich, D., et al. 2013, ApJS, 208, 23

Lee, H.-T., Takami, M., Duan, H.-Y., et al. 2012, ApJS, 200, 2

Massey, P., \& Johnson, J. 1993, AJ, 105, 980

Meaburn, J., \& Walsh, J. R. 1986, MNRAS, 220, 745

Medina, S.-N. X., Arthur, S. J., Henney, W. J., Mellema, G., \& Gazol, A. 2014 MNRAS, 445, 1797

Megeath, S. T., Cox, P., Bronfman, L., \& Roelfsema, P. R. 1996, A\&A, 305, 296

Meynet, G., \& Maeder, A. 2003, A\&A, 404, 975

Muzerolle, J., Calvet, N., \& Hartmann, L. 1998, AJ, 116, 2965

Ngoumou, J., Preibisch, T., Ratzka, T., \& Burkert, A. 2013, ApJ, 769, 139

O’Dell, C. R., Wen, Z., \& Hu, X. 1993, ApJ, 410, 696

Offner, S., Robitaille, T., Hansen, C. E., McKee, C. F., \& Klein, R. I. 2012 ApJ, 753, 98

Ohlendorf, H., Preibisch, T., Gaczkowski, B., et al. 2012, A\&A, 540, A81

Pekruhl, S., Preibisch, T., Schuller, F., \& Menten, K. 2013, A\&A, 550, A29

Preibisch, T., Hodgkin, S., Irwin, M., et al. 2011b, ApJS, 194, 10

Preibisch, T., Ratzka, T., Kuderna, B., et al. 2011a, A\&A, 530, A34

Preibisch, T., Roccatagliata, V., Gaczkowski, B., \& Ratzka, T. 2012, A\&A, 541, A132

Preibisch, T., Schuller, F., Ohlendorf, H., et al. 2011c, A\&A, 525, A92

Preibisch, T., et al. 2011d, A\&A, 530, A40

Probst, R. G., et al. 2008, Proc. SPIE, 7014 70142S

Povich, M. S., Smith, N., Majewski, S. R., et al. 2011a, ApJS, 194, 14

Povich, M. S., Townsley, L. K., Broos, P. S., et al. 2011b, ApJS, 194, 6

Rathborne, J. M., Brooks, K. M., Burton, M. G., Cohen, M., \& Bontemps, S. 2004, A\&A, 418, 563

Rathborne, J. M., Burton, M. G., Brooks, K. J., et al. 2002, MNRAS, 331, 85

Reed, B. C. 2003, AJ, 125, 2531

Reiter, M., \& Smith, N. 2013, MNRAS, 433, 2226

Reiter, M., Kiminki, M. M., Bally, J., Anderson, J., \& Smith, N. 2015, MNRAS, in press

Retallack, D. S. 1983, MNRAS, 204, 669 
Robitaille, T. P., Whitney, B. A., Indebetouw, R., \& Wood, K. 2007, ApJS, 169,328

Robitaille, T. P., Whitney, B. A., Indebetouw, R., Wood, K., \& Denzmore, P. 2006, ApJS, 167, 256

Roccatagliata, V., Preibisch, T., Ratzka, T., \& Gaczkowski, B. 2013, A\&A, 554, A6

Sanchawala, K., Chen, W.-P., Ojha, D., et al. 2007, ApJ, 667, 963

Sanduleak, N., \& Stephenson, C. B. 1973, ApJ, 185, 899

Smith, N., Bally, J., \& Brooks, K. J. 2004a, AJ, 127, 2793

Smith, N., Barbá, R. H., \& Walborn, N. R. 2004b, MNRAS, 351, 1457

Smith, N., Egan, M. P., Carey, S., et al. 2000, ApJ, 532, 145

Smith, N., Stassun, K., \& Bally, J. 2005, AJ, 129, 888

Smith, N. 2006a, ApJ, 644, 1151

Smith, N. 2006b, MNRAS, 367, 763

Smith, N., \& Brooks, K. J. 2007, MNRAS, 379, 1279
Smith, N., \& Conti, P. S. 2008, ApJ, 679, 1467

Smith, N., Bally, J., \& Walborn, N. 2010a, MNRAS, 405, 1153

Smith, N., Povich, M. S., Whitney, B. A., et al. 2010b, MNRAS, 406, 952

Stanke, T., McCaughrean, M. J., \& Zinnecker, H. 2000, A\&A, 355, 639

Stanke, T., McCaughrean, M. J., \& Zinnecker, H. 2002, A\&A, 392, 239

Tapia, M., Roth, M., Bohigas, J., \& Persi, P. 2011, MNRAS, 416, 2163

Tielens, A. G. G. M., \& Hollenbach, D. J. 1985, ApJ, 291, 722

Townsley, L., Broos, P. S., Corcoran, M. F., et al. 2011, ApJS, 194, 1

Walborn, N. R., Howarth, I. D., Lennon, D. J., et al. 2002, AJ, 123, 2754

Wang, J., Feigelson, E. D., Townsley, L. K., et al. 2011, ApJS, 194, 11

Wolk, S. J., Broos, P. S., Getman, K. V., et al. 2011, ApJS, 194, 12

Wünsch, R., Jachym, P., Sidorin, V., et al. 2012, A\&A, 539, A116

Yirak, K., Foster, J. M., Hartigan, P., et al. 2013, HEDP, 9, 251

Yonekura, Y., Asayama, S., Kimura, K., et al. 2005, ApJ, 634, 476

Zacharias, N., Finch, C. T., Girard, T. M., et al. 2013, AJ, 145, 44 